


\section{UNITED STATES OF AMERICA}

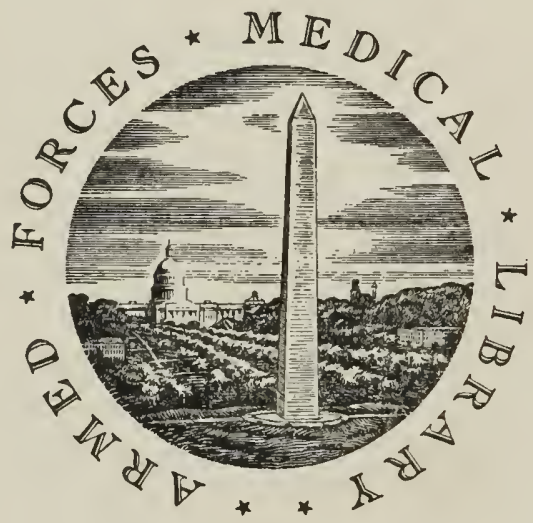

FOUNDED 1836

W A S H I N G T O N, D. C.

GPO $\quad 16-67244-1$ 

Hosack

ELGIN BOTANIC GARDEN- 

Scientific Bouks

A. E. FOOTE.

4

odech 


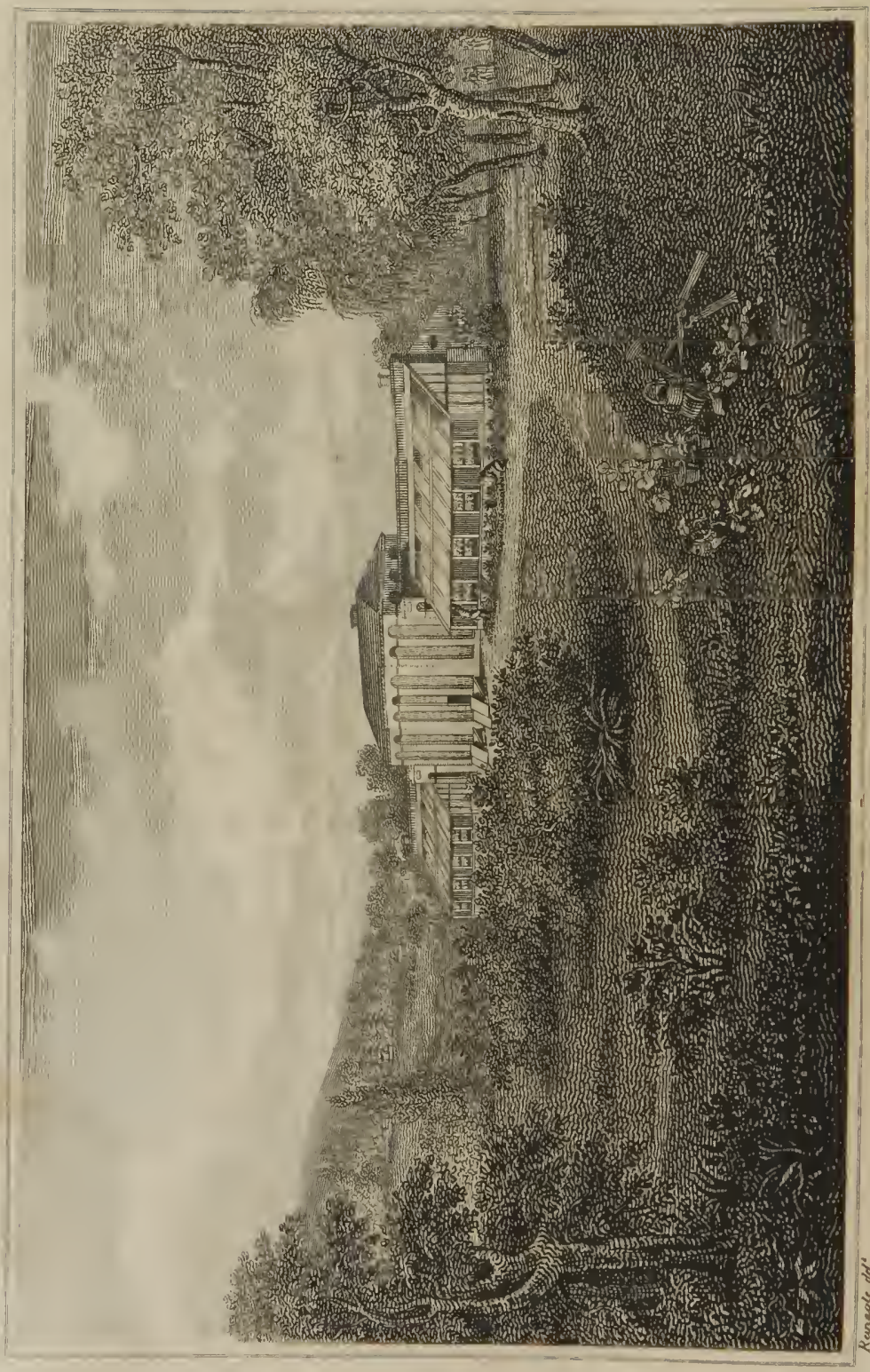




\title{
HORTUS ELGINENSIS:
}

\author{
OR \\ A CATAlogue \\ OF \\ P L A N T S,
}

INDIGENOUS AND EXOTIC,

CUTTIVATED IN THE

\section{ELGIN BOTANIC GARDEN,}

IN THE VICINITY OF THE CITY OF NEW-YORK.

\author{
ESTABLISHED IN 1801,
}

BY DAVID HOSACK, M.D. F.I.S.

Professol of Botany and Materia Medica in Columbia College,

Member of the American Philosophical Suciety, \&c.

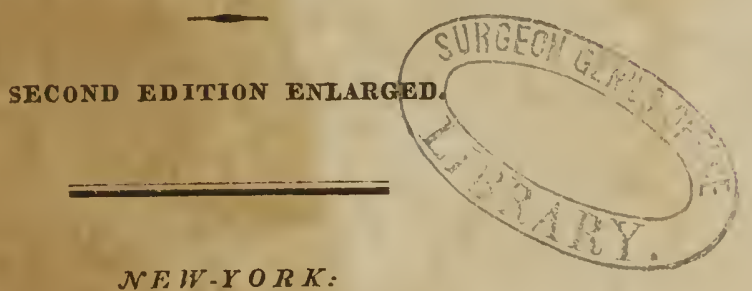

Pinted by T. E J. Szords, Printers to the Faculty of Physic

nf Columbia College, No. 160 Pearl-Street. 

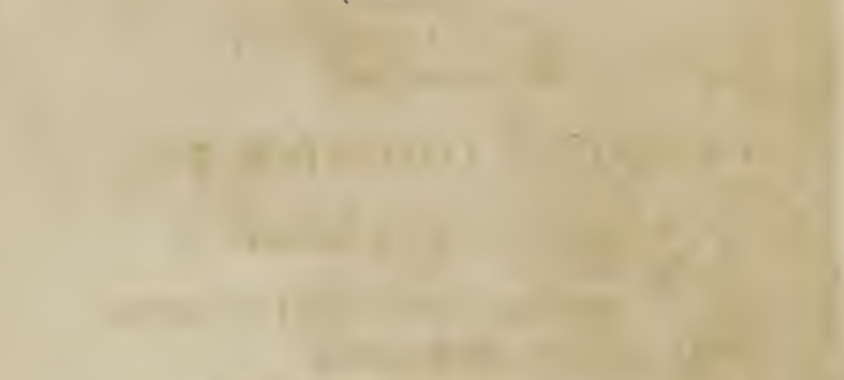

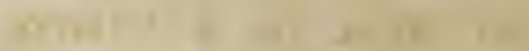

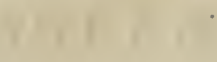$$
\text { (2) }
$$

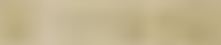

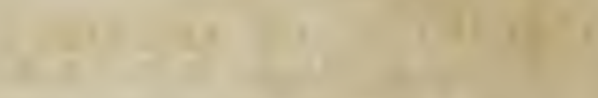

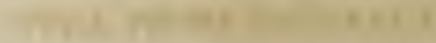
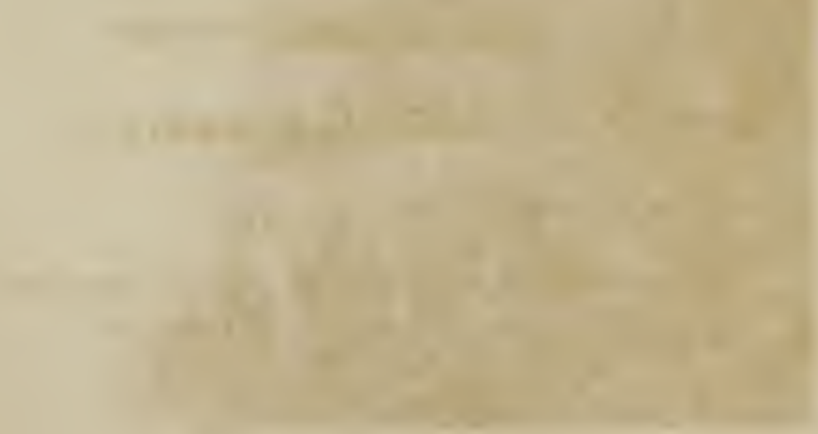
HIS EXCELLFNCY

\section{DANIEL D. TOMPKINS, Esq.}

Governor of the State of New-York;

THE HONOURABLE

\section{THE COUNCIL OF REVISION;}

$$
\text { AND }
$$

THE MEMBERS OF THE

\section{SENATE AND HOUSE OF ASSEMBLY,}

TIE PRESENT EDITION OF THE

\section{CATALOGUE OF PLANTS}

CULTIVATED IN THE

EIGIN BOTANIC GARDEN,

IS RFSPECTEULLY INSCRIBED. 

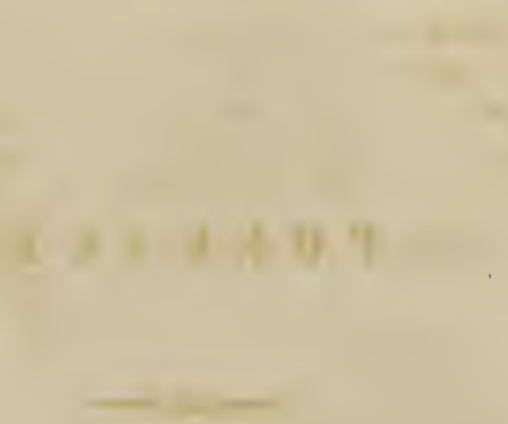

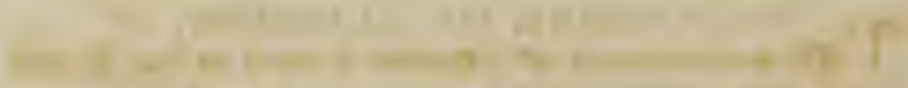

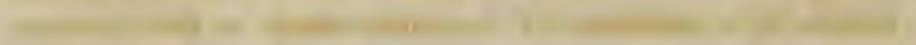

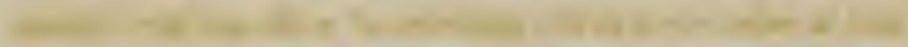

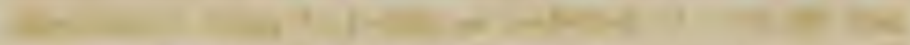

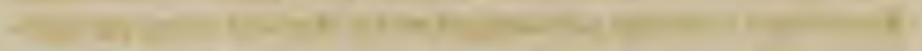
a

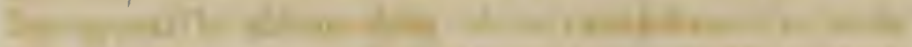

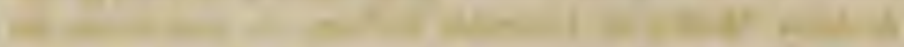

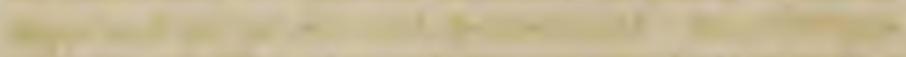

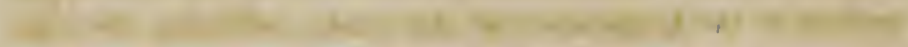

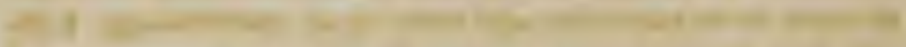

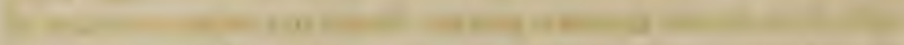
and 年

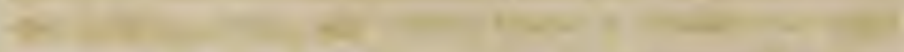
(1)

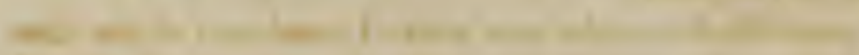
1

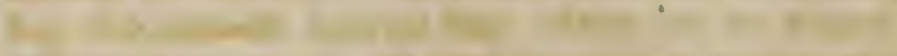

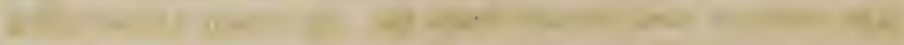

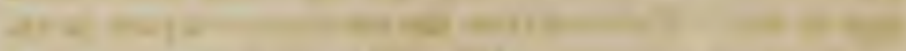

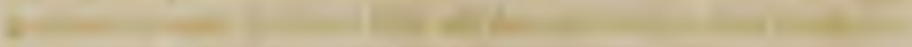

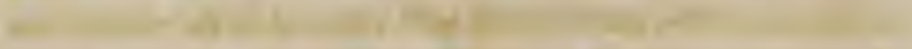

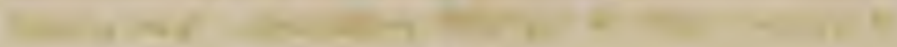




\section{P R E F A C E。}

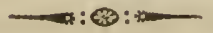

\section{$\mathrm{T}$}

IIE establishment of a Botanic Garden in the United States, as a repository of the native plants of this country, and as subservient to the purposes of medieine, agrieulture, and the arts, is doubtless an object of great importanee. Impressed with the advantages to be derived from an institution of this nature, I have anxiously endeavoured ever since my appointinent to the professorship of Botany and Materia Medica in Columbia College, to aceomplish its establishment. Disappointed, however, in my first applieations to the Legislature of this State, soliciting their assistance in so expensive and arduous an undertaking, I resolved to devote my own private funds to the proseention of this object; trusting, that when the nature of the institution should be better, and more generally known, and its utility fully ascertained, it would receive the patronage and support of the public.

Accordingly, in the year 1801, I purchased of the Corporation of the city of New-York, twenty acres of ground, situated on the midlle road between Bloomingdale and Kingsbridge, and distant from the eity about three miles and an half: 'The view from the most elevated part, is variegated and extensive, and the soil itself of that diversified nature, as to be particularly well adapted to the cultivation of a great variety of regetable productions. The grveater 
part of the ground is at present in a state of promising cultivation, arranged in a manner the best adapted to the difierent kinds of vegetables, and planted agreeably to the most approved style of ornamental gardening. Since that time, an extensive conservatory, for the more hardy green house plants, and two spacious hot houses, for the preservation of those which require a greater degree of heat, the whole exhibiting a front of one hundred and eighty feet, have been erected, and which, experience has shown, are well calculated for the purpose for which they were designed. The whole establishment is surrounded by a belt of forest trees and shrubs, both native and exotic, and these again are enclosed by a stone wall, two and an half feet in thickness, and seven feet in height.

As it has always been a primary object of attention to collect and cultivate in this establishment, the native plants of this country, especially such as are possessed of medicinal properties, or are otherwise useful, such gardeners as were practically acquainted with our indigenous productions, have been employed to procure them: how far this end has been attained, will be best seen by an examination of the Catalogue.

Although much has been done by the governments of Great-Britain, France, Spain, Sweden, and Germany, in the investigation of the vegetable productions of America: although mueli has been accomplished by the labours of Catesby, Kalm, Wangenheim, Schoepf, Walter, and the Michanx; and by our countrymen Clayton, the Bartrams, Colden, Muhlenberg, Marshall, Cutler, and the learned Professor Barton of Pennsylvania, much yet remains to be done in this western part of the globe. The numcrous articles of medicine which this country has already furnished; the variety of soils and climates which it comprehends, encourage the belief, that many more remain to be discovered, and that the Materia Medica may still be enriched by the addition of many indigenous plants, whose virtues yet remain undiscovered. 
Another object of importance is, to afford to students of medieine, the means of aequiring a knowledge of the natural history of plants, and the principles of botanie arrangement; a scienee intimately connected with their profession, as it not only enables them to distinguish one plant from another, but fiequently leads to an aequaintance with their medicinal virtues. For this purpose the grounds are divided into different compartments, calculated to exhihit the various plants according to their several properties: and these again are so arranged as to afford a practieal illustration of the systems of botany at present most estecined, viz. the sexual system of Linnceus, and the natural orders of Jussieu.

Hitherto the botanical gardeus of Edinburgh, Oxford, Cambridge, London, Paris, Copenhagen, Leyden, Upsal, Goettengen, \&ce. liave instrueted the Ameriean youth in this department of medical education; and it is in some degree owing to those establishments that the universities and colleges of those places have become so eclebrated, and have been resorted to by students of medicine from all parts of the world.

Since the publieation of the first edition of this eatalogne. in 1806, this institution has been greatly improved, and by an act of the Legislature, passed on the 12th day of Mareh, 1810, has beeu purehased by the State for the benefit of the Medical Schools of New-York. For information upon this subjeet the reader is referred to a production entitled, "A Statement of Facts relative to the Establishment and Progress of the Elgin Botanic Garden, and the subsequent disposal of the same to the State of Nerw-York," just published; in which the liberal and enlightened views of the Legislature, in making so large and honourable an appropriation for the promotion of seience are more fully exhibited than would be proper upon this oceasion. It will also be pereeived by a comparison of the present with the former edition, that very considerable additions have been made to the collection both of the foreign and indigenons 
plants contained in that establishment. Gratitude demandis of me, on this occasion, an acknowledgment of the obligations I am under to many distinguished botanists, both abroad and at home, who have contributed to this institution. In this number are to be enumerated my much esteemed and respeeted fier $\mathbf{l}$ and instructor, Dr. James $\boldsymbol{E} d$ rourd Smith, the learned President of the Linnæan Society of London; the late Professor Vahl, and Mr. Hoffmar Bang, of Copenhagen; Mons. Desfontuines and Thouin, the celebrated Professors of Botany and Agriculture at the Medical Sehools of Paris; Mr. Salisbury, Proprietor of the Botanie Garden at Brompton, near London; the late Dr. Fabroni, Direetor of the Royal Museun of Florence; Dr. Bostock, the learned President of the Botanic Institution of Liverpool; Dr. Lettsom, of London; Dr. Andvere Michaux, Editor of the Flora Boreali Americana, and Author of the very valuable History of the Forest Trees of North-America, now publishing at Paris; my much esteemed friend Dr. Alive Raffinean Delile, of the Institute of Egypt; Dr. Alexander Anderson, Superintendant of the Botanic Garden at St. Vincents; and Baron De Schach, of Martinique. From these gentlemen I have reeeived many rare botanical works, and some of the most valuable plants in this colleetion.

Nor must I be unmindful of the obligations I am under vo screral grentlemen in this country, distinguished for their taste and talents in this department of science. The Hon. Robert R. Livingslon, our former Minister in France; Professor .Nitchill, of this city; John Stevens, Esq. of Hoboken; Mr. Revnard JF.Nahon, of Philadelphia; Mr. Stephen Elliot, of Beaufort, South-Curolina; Dr. Darlington, and Mr. John Vaughan, of I'ennsylvania; John Le C'onte, Esu. of Georgia; Mr. Willicim I'rince, of Long-Island; and Nir. Andrew Gentle, setsman, of this city; are also among the contributors to this institulion. It is but justice to the merit of my nephew, Dr. Caspar Wistar Eddy, a young but accurate botanist, to atid, that he has largely aug: 
mented the collection of American plants, especially of those of the island of New-York; some of which, viz. two new species of Gerardia, were first discovered by him in the vieinity of this eity. From my other pupils now industriously proseeuting the study of botany and medicine, more especially Mr. John W. Francis, and Mr. Isauc Roosevelt, of this city, and Mr. Robert M. Barclay, of Orange county, I also anticipate many fruits of their labours in this department of seicnce.

It would be injustice to my late gardener, Mr. Frederich Pursh, who with a knowledge of the science of botany unites a very extensive and aceurate aequaintanee with the plants of this country, not to notice the very nunerous contributions he has made to the collection, of the native plants of the United States, during the period he had cliarge of this establishment. The institution is also at present, and has been for some months past, in a very flourishing condition, under the direction of Mr. Dennison, who has been very particularly recommended to me by Messrs. Lee and Kennedy, of Hainmersnith. The present. state of the collcetion is an evidence of his attention and skill, and from which I expect great improvements in every part of the establishment.

I avail myself of this oceasion to observe, that as soon as measures may be taken by the Regents of the University for the permaneut preservation of the Botanic Garden, it is iny intention immediately to commence the publication of American Botury, or a Flora of the United States. In this work it is my design to give a deseription of the plant, noticing its essential eliaracters, synonyms, and place of growth. with observations on the uses to which it is applied in medicine, agrieulture, or the arts; to be illustrated by a coloured engraving, in the same manner in which the plants of. Great-Britain have been published by Dr. J. E. Sinith, in his English Botany. Considerable progress lias already been made in obtaining materials for this publication : many of the drawings will be excented by Mr. James Indervick, 
a young gentleman of great genius and taste, and others by John Le Conte, Esq. whose aequaintance with botany and natural history in general will enable him to execute this part of the work with great fidelity. In Mr. Pursh, whose name has already been mentioned, I shall have a very industrious and skilful botanist to collect from different parts of the union such plants as have not yet been assembled at the Botanic Garden.

D. H.

New-York, March 12th, 1811. 


\section{ABBREVIATIONS.}

\begin{tabular}{|c|c|c|}
\hline & 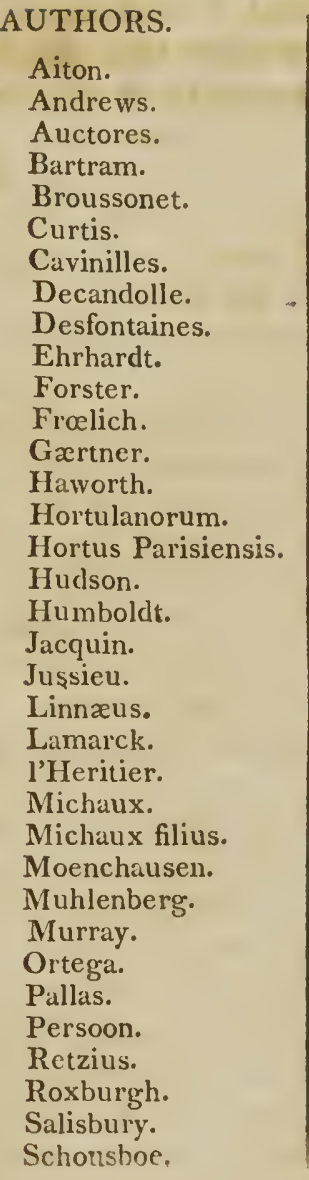 & \begin{tabular}{|ll} 
Schr. & Sc \\
Scofi. & Sc \\
Sm. & Sm \\
Swz. & Sw \\
Thunb. & Th \\
Vent. & Ve \\
Wald. & W \\
Walt. & W \\
Wang. & W \\
Wend. & W \\
Willd. & W
\end{tabular} \\
\hline
\end{tabular}


It is very probable, that owing to the engagements, professional and others, with which I am occupied, that some errors may be found in the following Catalogue; but I trust they are few in number, as it has been printed under the constant inspection of my nephew before mentioned.

D. $H$. 


\section{HORTUS ELGINENSIS.}

English Name. Nat.country. use. cult clurat. ABROMA, or false cacou

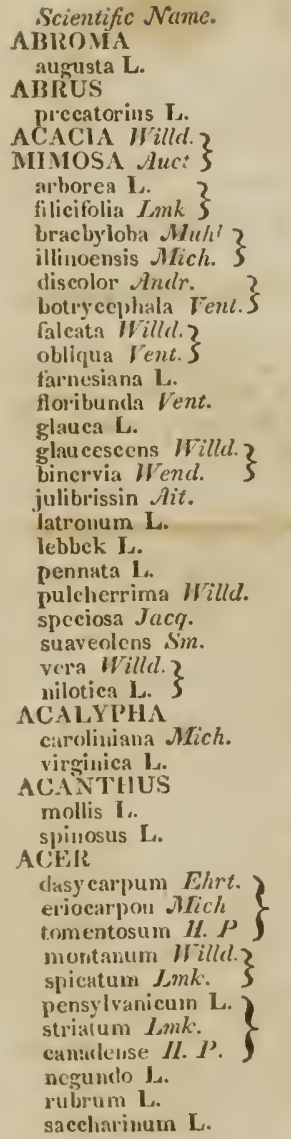

maple leaved
VILD LIQUORICE
Jamaica

ACACIA

\begin{tabular}{|c|c|c|c|}
\hline tree & WV.Indies & & h.h \\
\hline short podded & N. Amer. & & $\mathbf{h}$ \\
\hline two coloured & N. II oll. & & $\mathrm{g} \cdot \mathrm{h}$ \\
\hline sickle leaved & do. & & $\mathrm{g} \cdot \mathbf{h}$ \\
\hline $\begin{array}{l}\text { Farncsian. Sfonge-tree } \\
\text { fowering } \\
\text { glaucous }\end{array}$ & $\begin{array}{l}\text { WV. Inties } \\
\text { N. Holl. } \\
\text { S. Amer. }\end{array}$ & & $\begin{array}{l}\text { h.h } \\
\text { g.h } \\
\text { h.h }\end{array}$ \\
\hline two nerved & N. Holl. & & $\mathrm{g} \cdot \mathbf{h}$ \\
\hline $\begin{array}{l}\text { oriental } \\
\text { robbers } \\
\text { Egyptian } \\
\text { feathered } \\
\text { beautiful } \\
\text { shewy } \\
\text { sweet secnted, }\end{array}$ & $\begin{array}{l}\text { Levant } \\
\text { F. Indies } \\
\text { Egypt } \\
\text { E. Indies } \\
\text { S. Amer. } \\
\text { E. Indies } \\
\text { N. I Ioll. }\end{array}$ & & $\begin{array}{l}\text { h } \\
\text { h.h } \\
\text { h.h } \\
\text { h.h } \\
\text { h.h } \\
\text { h.h } \\
\text { g.h }\end{array}$ \\
\hline gum-arabic tree & Egypt & Ned. & h.h \\
\hline $\begin{array}{l}\text { CALYPHA } \\
\text { Carolinian } \\
\text { common } \\
\text { EAR'S BREECH }\end{array}$ & $\begin{array}{l}\text { N.Amer. } \\
\text { do. }\end{array}$ & & $\begin{array}{l}\text { h } \\
\mathbf{h}\end{array}$ \\
\hline $\begin{array}{l}\text { ofi. leaved } \\
\text { ginous }\end{array}$ & $\begin{array}{c}\text { Europe } \\
\text { dlo. }\end{array}$ & Med. & $\begin{array}{l}\text { h } \\
\text { h }\end{array}$ \\
\hline
\end{tabular}
E. Indies
h.h s
B. Indies
h.h s

\section{silver lcaved, or soft}

N. Amer. Arts

shrubby, or mountain

striped barked

ash leaved

common red

sugar.

\section{ACH.ANIA}

ACIIA NIA

malvavicus Sivz.

Ilibiscus malvaviseus L. T mallow leaver?

Malraviseus aboreus bashtul mallow

$\begin{array}{llll}\text { do. } & & \text { h } & \text { s } \\ \text { do. Arts } & \text { h } & \text { t } \\ \text { do. } & & \text { h } & \text { s } \\ \text { do. } & \text { Arts } & \text { h } & \text { t } \\ \text { do. } & \text { Nits } \& & \text { h } & t \\ & \text { Diet } & & \end{array}$

IV. Inrlies

h.h 5 


\section{ACHILLEA}

asplenifolia Vent.?

rosea $\boldsymbol{H} . \boldsymbol{P}$

decumbens $L m k$.

millefolium $\mathbf{L}$.

ptarmaca L

v. fore pleno

tanacetifolia $\mathbf{L}$.

tenuifolia $L m k$. santolina Willd.

ACHRAS

Sapota L.

ACHYRANTHES argentea $L m k$.

ACNIDA

camnabina $\mathbf{L}$.

ACONITUM

napellus $\mathbf{L}$.

ACORUS

calamus L.

ACTIEA

racemosa $\mathbf{L}$.

spicata $\mathbf{L}$

ADENAN'THERA

pavonina $\mathrm{L}$.

ADI A NTUM

pedatum L.

ADONIS

restivalis $\mathbf{L}$.

autumnalis $\mathbf{L}$.

EGILOPS

triuncialis $\mathbf{L}$

EGLE

marmelos Correa.

Cratæva marmelosL. $\}$

ESCHYNOMENE asnera L.

\section{ESCULUS}

hippocastanum, L.

fiva Ait.

lutea Wang. $\}$

macrostacliy: Nich.
parvilora Walt. pavia L.

$\mathrm{AGAVF}$

americana L.

v. variegata

tuberosa Ait.

virginica $\mathbf{L}$.

vivipara $\mathbf{L}$.

AGRIMONIA

eupatoria $\mathbf{L}$.

narviflora Ait.

AGROSTEMMA

colonaria L.

gitlıgo L.

Githago segeturn Desf.

AGROSTIS

alba $\mathbf{L}$.

canina $\mathbf{L}$

\section{HORTUS ELGINENSIS.}

YARROW, or milfoil

rose flowering

trailing

common

sneeze wort

variety

tansey leaved

fine leaved

SAPOTA

common, sour sop

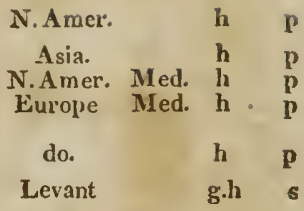

W. Indies Diet \& g.h s Med.

CHAFF FLOWER

silvery

WILD HEMP

common

WOLF'S BANE

common

SWEET FLAG

aromatic calamus

HEIRB. CHRISTOPHER

black snake root

common, coral and pearl

PEACOCK FLOWER

smooth leaved

MAIDFN HAIR

American

C. G. H.

b.h a

N. Amer.

$$
\text { h }
$$

Europe h p

N.Amer. Med. li p N. Amer. Med.
do.

B. Indies

h.h s

FLOS ADONIS, or pheasant's eye

summer

autumnal

HARD GRASS

long spiked

MARMELADE APPLE

common

Europe

do.

Europe

E. Indies Diet \& h.h s

ESCHYNOMENE, Bastard sensitive plant rough

HORSE CHESNUT

common

yellow flowered

E. Indies

h.h a

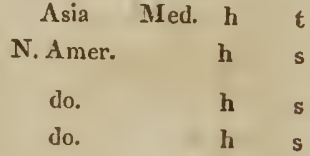

long spiked

searlet flowering

AGAVE

A merican

variegated, do

tuberous

Virginian

viviparous

AGRIMONY

conmon

small flowered

ROSE CAMIPION

common

corn cockle

BENT GRASS

marsl or white Herd's grass. Penn.

brown
S. Amer. Arts g.h s do.

N. Amer.

g.h s

S. Amer. g.h s

N.Amer. Med. \& h p

do. Arts h $\mathrm{l}$

Europe $\mathbf{b}$ b

do. h

do. Agr. in P

de. Agr. h p 
HORTUS ELGINENSIS.

compressa Willd.

dubia Iseers.

miliacen L.

littoralis $\mathrm{S} m$.

mexicana $\mathrm{L}$.

rupestris Hilld.

setacen $\mathrm{Sm}$.

spica venti $L_{\text {. }}$

stricta IVilld.

virginica $\mathbf{L}$.

vulgaris Sim.

hispida Willd.

capillaris Leers.

AIRA

aquatica $\mathbf{L}$.

canescens $\mathbf{L}$.

caspitosa $\mathbf{L}$.

maryopliyllea L.

Aexuosa $\mathrm{L}$.

melicoides Mich.

olstusata Mich.

praecox $\mathbf{L}$.

1I. CIEMILLA

liybrida 1.

pubescers $\mathrm{rmk}$. $\}$

IIE'IRIS

fariuosa l. 2

alba Mich.

ALISMA

plantago $\mathrm{L}$.

v. minor

small

millet:

sea

Mexiean

rock

bristly

silky

upiright

barren

common

IIAIR GRASS

water.

grey

turfy

silvery

heatli, or zigzag

melic like

blunt

spring

LADIES MANTLE

Europe

ilo.

do.

S. Amer.

Europe

h

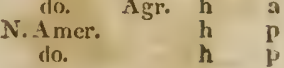

Europe $A$ gr. h

hairy

ALETIRIS

mealy; Deril's bit, stargrass

WATER PLANTAIN

common

lesser, do

ALLAMANDA

cathartica $L$.

Orelia grandiflora $A u b l$.$\} purging; willow leaved$

ALLIUNI

ascalonicum L.

canadense L.

cepa L.

fistulosum $\mathbf{L}$.

moly L.

oleraceum $\mathbf{L}$.

porrum L.

sativum L.

sclıncenoprasum $\mathbf{L}$. senescens $\mathbf{L}$.

ALNUS

serrulata Hilld.

Betula rugosa Ehrt.

AL.OE

fruticosa Pers.
arbor'escens . Hill.

ling 11 a $\mathrm{L}$.

maregaritifera 1.2

pumili Thunb. $\}$

perfoliata L. $\mathbf{L}$

picta ' $I \%: u$ unb.

maculosa lank. umbellata Decand.

retusa I.

\section{ONION GARLICK}

eschallots

wild, or $\Lambda$ merican

common onion

Welch do

yellow do

purple striped do

commion leck

common garlick

chives

narcissus leaved

A LDER

common American

A LOE

tree

tongue

pearl

great soap, succotorine

spotted soap

cusbion; thumb
S. Amer. h.h s

Levant Dirt h p

N. Amer. Diet h p

Diet \& h p

Siberia I Diet h p

Europe Diet

do. Diet h p

do. Diet \& h b

do. Diet \&

Med. h $p$

Siberia Diet h p

do. Diet h p

N.Amer, h s

C. G. H. $\quad$ s.h $\mathrm{s}$

do. g.h s

do. g.h s

Africa Med. g.h s

C. G. H. g.h 8

clo. g.h s 
IORTUS ELGINLNSIS.

spiratis $\mathbf{L}$. variegata $\mathbf{L}$. veriucosa Ait.

ALOPECURUS

bulbosus $\mathbf{L}$.

genieulatus L. pratensis $\mathbf{L}$.

ALSINE

media $\mathbf{L}$.

ALSTRAEMERIA

ligtu $L$.

ALTH症A

fieifolia Willd.

Alcea ficifolia L. $\}$

oflicinalis $\mathbf{L}$.

rosea Car.

Alcea rosea L. $\}$

ALYSSUM

clypeatum L.

incanum L.

saxatile $\mathbf{l}$.

utriculatum L.

AMARAN'THUS

albus $\mathrm{L}$.

blitum L.

cruentus $\mathbf{L}$.

liybridus L.

retrofiexus $L m k$. $\}$

hypochondriacus $\mathbf{L}$.

lividus L.

molancholicus $\mathbf{L}$.

oleracens L.

paniculatus $\mathbf{L}$.

sanguineus $L$.

scaidens $\mathbf{L}$.

spinosus $\mathbf{L}$.

tivicolor $\mathbf{L}$.

tristis $\mathrm{I}$.

AMARYLLIS

atamaseo L.

aurea l'Jler?

belladuna $\mathbf{L}$.

equestris Ait.

formosissima L.

longifolia $\mathbf{L}$.

lutea $\mathbf{L}$.

ornata Jit.

reginæe $\mathbf{L}$.

sarvieusis $\mathbf{L}$.

nuclulata $\mathbf{L}$.

vittuta . Hit.

AMBROSIA

artcunisiefolia $L$.

elatior 1.

tritida $\mathbf{L}$.

AMOMUN

zingiber L.

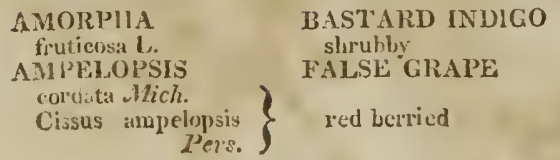

partridge breast

FOX TAIL GRASS

bulbous

floating

meadow

CHICK WEEU

cornmon

ASTROEMERIA

striped flowered

MARSH MALLOW

fig leared holly hook

common

common holly hock

MADIVOR'T

hoary

rock

bladder

white

strawberry

various leaved

clustered

Prince's feather

lead coloured

Love lies blecding

cultivated

panicled

bloorly

climbing"

thorny

tliree coloured

mourning

AMARYLIIS

Atamasco lily

golden

Belladonna bily

J3arbartoes fily

Jacobrea lily

long leaved

yellow

Cape coast lily

Mexican lily

Guernsey lily

wared leaved

ribbon

rough

snioath

hemp

GINGER

true, narrow leared
C. G. H.

do.

do.

$\begin{array}{ll}\text { g.h } & \text { s } \\ \text { g.h } & \text { s } \\ \text { g.h } & \text { s }\end{array}$

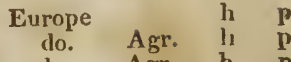

do. Agr. h p

N.Amer. Dict h a

Peru

g.h P

Levant

Europe Med. h l'

China Med. h b

shield sceded , Europe

Levant

do.

AMARANTII or Cock's comb

N.Amer.

Europe

Clina

N. Amer.

do.

do.

F. Indies do.

N.Amer.

IV. Indies

S. A mer

13. Indies

E. Indies do.

h a

h p

g.h $\mathrm{p}$

g.h P

h a

h a

h $\mathrm{a}$

h a

h a

ha

h a

Dict h a

h a

h a

h a

h a

ha

h a

Carolina

Peru

S. Amer.

W. Indies

S. A mer.

C. G. H.

S. Europe

Guinea

II exico

Japan

C. G. $\mathrm{H}$.

do.

$\begin{array}{ll}\text { g.h } & \text { p } \\ \text { h.h } & \text { p } \\ \text { g.h } & \text { p } \\ \text { h.h } & \text { p } \\ \text { g.h } & \text { p } \\ \text { g.h } & \text { p } \\ \text { g.h } & \text { p } \\ \text { h.h } & \text { p } \\ \text { g.h } & \text { p } \\ \text { g.h } & \text { p } \\ \text { g.h } & \text { p } \\ \text { g.h } & \text { p }\end{array}$

N. Amer.

do.

do.

h a

E. Incies Med.\& h.h p Diet

N. Amer. Arts h s

do.

h s 
HORTUS ELGINENSIS.

quinquefolia Mich.

Herlera quinquefolia L. Wild iry

N. Amer.

hs

bipinnata Mich.

Vitis arborea $\mathbf{L}$.

Cissus stans Pers.

AMYGDALUS

communis L.

nana L. flore pleno

persica L.

v. nuci persica

v. fore pleno

ANACARIIUM

occiflentale $\mathbf{L}$.

ANAGALLIS

arvensis $\mathbf{L}$.

v. fore coeruleo

v. Aore phoniceo

A NCIIUSA

augustifnlia $\mathbf{L}$.

officinalis $\mathrm{L}$.

sempervirens $L$.

ANIROMED A

axillaris $1, m k$. $\}$

catesbaei $\mathbf{W a}$ calrculata $\mathbf{L}$.

ferruginea Ait.

mariana $\mathbf{L}$.

nitilla Bartr.

lucirla $\mathrm{Lmk}$.

coriacea Ait.

panieulata Ait. $\}$

racemosa Lmk.

upright

do.

h s

ALMOND

common

dwarf double flowering

common peach

nectarine dlo.

doulle flowering do.

CASHEI NUT

common

Barbary

Tartary

$\begin{array}{ll}\text { h } & t \\ \text { h } & \text { t } \\ \text { h } & t \\ \text { h } & t \\ \text { h } & \text { t }\end{array}$

PIMPERNEL, Chick-oweed

common

blue flowered

rell flowered

BUGLOSS

narrow leaved

common or officinal

evergr'een

ANDROMEDA

saw leaved

box leaved

ferrugineous

oval leaved

shining leaved

B. Indies Diet\& h.h s

Med.

polifolia L. Lubuluta Pers. $\}$

racemosa $\mathbf{L}$.

paniculats Walt. $\}$

speciosa Mich. ?

cassinefolia Vent. $\}$

v. pulverulenta Bartr.

ANIBOPOGON

sclocenantlus $\mathbf{L}$.

scoparium Mich.

virginicum $\mathbf{L}$.

ANDRYALA

cheiranthifolia l'Her.

glandulosa $L m k$. tomentosa Scop.

ANEMONE

aconitifolia Jfich. )

dichotoma $\mathbf{L}$.

pensylvanica L.

coronaria $\mathbf{L}$.

hepatica L.

v. flore pleno

lourtensis $\mathbf{L}$.

nemorosa $\mathbf{L}$.

thalictroides L.
Thalictiuin anemo-

panicled

rosemary leaved

sweet scented

cassine leaved

N. Amer. Med. h a

$\begin{array}{cccc}\begin{array}{c}\text { Europe } \\ \text { do. } \\ \text { do. }\end{array} & \text { Med. } & \begin{array}{c}\text { h } \\ \mathbf{h}\end{array} & \mathbf{p} \\ \mathbf{h} & \mathbf{p}\end{array}$

powdered

BEARD GRASS

Lemon grass

broom grass

yellow broom grass

ANDRYALA

\begin{tabular}{|c|c|}
\hline $\begin{array}{l}\text { N. Amer. } \\
\text { do. } \\
\text { do. } \\
\text { do. }\end{array}$ & a \\
\hline do. & \\
\hline do. & \\
\hline do. & \\
\hline do. & \\
\hline do. & \\
\hline $\begin{array}{l}\text { E. Indies } \\
\text { N. Amer. } \\
\text { do. }\end{array}$ & Med. 1 \\
\hline
\end{tabular}

A

stock leaved

Madeira

g.h P

ANEMONE-Wind forver

ข. fiore pleno

rirginians 1.

wolf's bane leaved

common

hepatica-liver zort

double flowering

garden

wood

rue leaved

N. Amer.

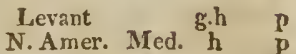

Europe h p

N. Amer. Med. h p

double flowered do.

crow foot leaved do.

h $p$

do.

h $n$ 
ANETHUM

foniculum L. graveolens $L$.

ANGELICA archangelica $\mathbf{L}$. triquinata. Hich.

\section{ANONA}

muricata L.

tripetala Ait.

eherimolia $L m k$. $\}$

ANTHEMIS

artemisize folia Willd.

grandiflora $H . P$.

stipulacea Moench.

Chrysanthemum indicum Curt.

cotula L.

nobilis $\mathbf{L}$.

tinctoria $\mathbf{L}$.

$\left.\begin{array}{l}\text { trilobata Ort. } \\ \text { Zaluzania trilobats } \\ \text { Pers. }\end{array}\right\}$

ANTHERICUM

frutescens $\mathbf{L}$.

ramosum $\mathbf{L}$.

Phalangium ramosum $\}$ branching

ANTHOLYZA

xthiopica $\mathbf{L}$.

cunonia $\mathbf{L}$.

ringens $\mathbf{L}$.

AN'THOXANTHUM odoratum $\mathbf{L}$.

ANTHYLLIS

barba jovis $\mathbf{L}$.

cretica $L m k$.

Ebenus cretica L. $\}$

hermannix Vahl.

Aspalathus cretica L. $\}$

vulneraria $\mathbf{L}$.

ANTHRIINUM

canadense $l$.

cymbalaria $\mathrm{I}$.

junceum $\mathbf{L}$.

Jinaria L.

majus L.

purpureum L. triphyllum L.

APIUM

graveolens $\mathbf{L}$.

petroselinum L.

APOCYNUM

androsemifolium $\mathbf{L}$.

cannabinum $\mathbf{L}$.

AQUILBGLA

canadensis $\mathbf{L}$.

vulgaris $\mathbf{L}$.

v. variegala

v. ft. pleno

Al IBIS

canadensis $\mathrm{L}$.

lyrata $\mathrm{L}$.

penduix I.

sweet

dill

common

dyers

scarlet

gaping

Canadian

wall

r'lish

toad flax

great

purple

CELERY

garden

conimon

small

tall
FENNEL

ANGELICA

garden or officinal

American or wilk]

CUSTARD APPLE

rough fruited

broad leaved

CHAMOMILE

artemisia leaved Chry- Clina

h p santhernum

Europe Med. h do. do. h a

Europe do. h b N. Amer. do. h p

S. Amer. h.h s

Peru Diet h.h s

stinking-May weed

N. Amer. Med. h

Europe Med.

do. Airts

tliree lobed

Mexico

g.h b

SPIDER WORT

shrubby C. G. H.

g.h p

Europe

h p

ETHIOPIAN CORN FLAG

orange coloured

VERNAL GRASS

sweet scented

KIDNEY VETCH

Jupiter's beard

black ebony

lavender leaved

common yellow

SNAP DRAGON

three leaved

- common parsley

DOG'S BANE

tutsan leaved

Indian hemp

COLUMBINE

scarlet or wild

variegated do.

coulile fowered

WALL ClRESS

Canadian
C. G. H.

do.

do.

$\begin{array}{ll}\text { g.h } & \text { p } \\ \text { g.h } & \text { p } \\ \text { g.h } & \text { p }\end{array}$

N. Amer. Agr. h p

Europe gh s

Crete Arts g.h $t$

Palest. g.h s

Europe $\quad$ h $p$

N. Amer. $\quad h \quad a$

Spain h

N. Amer. Med. h p

do

Barbary

Europe Jiet h b

do. Med.\& in is

Diet

N. Amer. h p

do.

h

N.Amer. h p

Europe h p

$\begin{array}{ccc}\text { N. Amer. } & \text { h } & \text { l? } \\ \begin{array}{c}\text { Europe } \\ \text { do. }\end{array} & \text { h } & \text { a } \\ & \text { h } & \text { a }\end{array}$
Furope $\quad h \quad p$ 
HORTUS ELGINENSIS.

ARACHIS

liypogrea $\mathrm{L}$.

ARILIA

hispida Mich.

nuclicaulis $\mathbf{I}$.

iracemosa $\mathbf{L}$.

spinosa 1.

Alzc'TIUM

lappa $\mathbf{L}$.

A IRIDISIA

solonacea $\boldsymbol{R} 0 x b$.

ARENARIA

eanadensis Pers.

rubia v. marina L.

rubra $\mathbf{L}$.

trinervia $\mathrm{J}$.

serpillifolia I.

A RETIIUSA

ophinglossoirles L.

ARGFIIUNE:

mixicana $\mathrm{l}$.

ARISTIDA

dichotoma Wich

A RISlOOI OCIII

odoratissima $\mathbf{L .}$ serpentaria $\mathbf{L}$.

ARTFilISI

abrotanum 1.

absynthiuin $\mathbf{L}$.

dractuculus $\mathrm{L}$.

AITOC.ARPUS incisa $\mathrm{L}$.

AliUM

dracontium $I$.

tripliyllum L.

ARUNDO

aremaria L.

epigejos L.

phragmitis 1.

AS.IRUMI

arifolium Mich.

canalense L.

ISCLEI'IAS

amona 1 .

curassavica L.

finticosa l.

hybrida aich.?

variegata $/ F$ all. $\}$

ill carvata L.

Jongifolia Jijch.

$\left.\begin{array}{l}\text { uigı L. } \mathrm{L} . \\ \mathrm{Cy} \text { uauchum nigrum } \\ \text { Pers. }\end{array}\right\}$

oltusifolia .Mich.

paupercula Mich.

pulchra Ehrt.

purpurascens L.

syriaca $\mathbf{L}$.

tubeinsa L.

decumbens Walt

verticillata $L$,

vintetoxicum L.

Cyuauchum vincetoxicum Pers.
GlROUNI or PEA NU'T

A merical

IV. Indies Diet h a

ARALIA

bristly

wild sarsaparilla

N. Amer.

do. Med. h

berry-hearing spikenard

angelic tree Pricklyash

BUHDOCK

conimon

AIRDISI I

uight shade flowering

do. do. $\mathrm{h}$

do.

do. h

N. Amer: do. b b

SANI) WORT

mar'sh

field or red

thiree nerved

thyine leaved

A]ETHUS 1

adder's tongue leaved

IOUNEID POPPY

Mexican

E. Indies h.h s

RISTIDA

small

BIRTH WORT-Snake-root

sweet scented

Virginian

WORMWOOJ

southernwood

commou

Tarrigon

BRFA I FRUIT TREE

South Sea

INDIAN 'L'URNEP

many leaved

three leaved

IREED GIRASS

sand

small

common

N. Amer: h a

Europe I

N. Amer. ho h

N. Amer. h p

Mexico h a

N. Amer. It P

IV. Inclies h.h s

N. Amer. Med. h p

Europe Med. h s

do. do. h p

Siberia Diet h

S. Sea Isl. Diet h.h t

N. Amer. Med. h p do. do. h p

N. Amer. h p

do. li i,

ISARABACCA-Mild ginger

heart snake root $N$. Amer. Med. Il p whice snake root do. do. I

SIVALIUIV IVORT-Silk weed, Milk weed

oval leaved

curassao

willow leaved

N. Anier.

IV. Indies

C. G. H.

h p

variegated

flesh coloured

N. A nier.

g.l

long leaved

do.

do.

i) $\boldsymbol{p}$

black

Furope Med. I

obtuse leaved

linear leaved

liver

purple

common

Pleurisy root. Butter-fly rweed

whorl'd leared

N. Amer.

do.

do.

do.

do. Arts

do. Med. It l'

do. h

officinaI

Furope Med. h p 


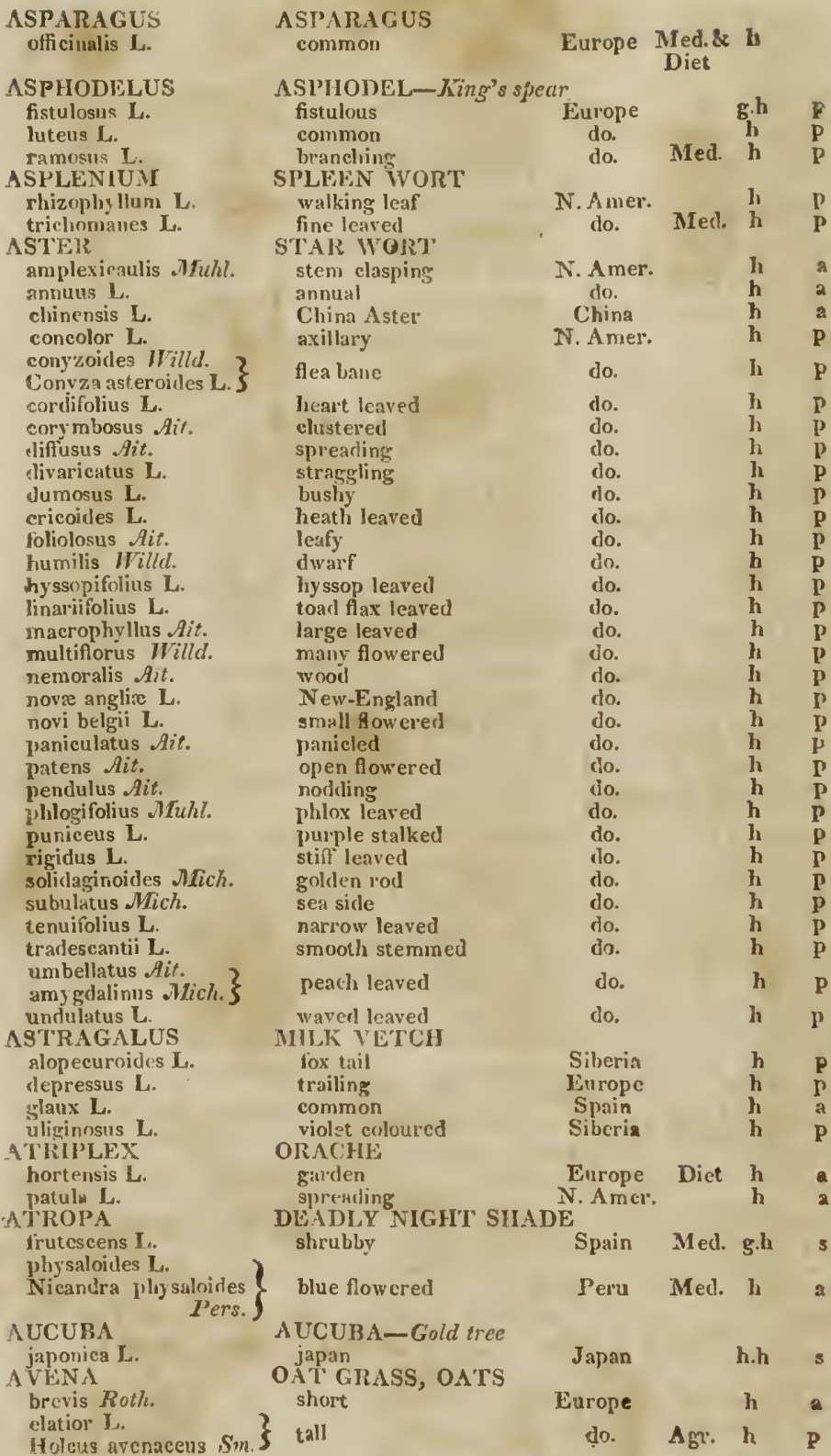




\section{HORTUS ELGINENSIS.}

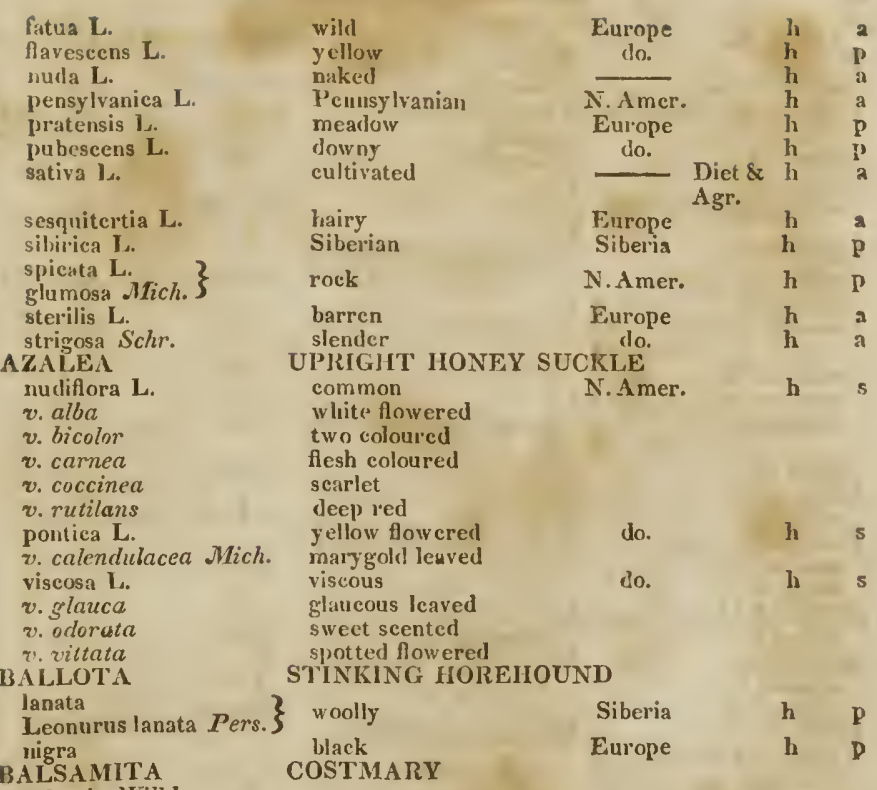

vulgaris Willd.

major Desf.

Europe Med. h p

BASELLA rubra L.

BAUHINIA

acuminata $\mathbf{L}$.

candida Ait.

divaricata $\mathbf{L}$.

porrecta Ait.

tomentosa $\mathbf{L}$.

variegata $L$.

BELLIS

perennis, $\mathbf{L}$.

BERBERIS

vulgaris, L. $\}$

v. canadensis

BETA

cicla $\mathrm{L}$.

v.

vulgaris $\mathbf{L}$.

BETONICA

officinalis

BETULA

alba $L$.

Ianulosa Mich. ?

nigra $\mathbf{L}$.

Ienta L. populifolia Ait. $\}$ ycuminata E.hrt. $\}$
MALABAR NIGHT SHADE

red, or Chinese Spinach E. Indies Diet h.h b

BAUHINIA, or Mlountain Ebony

sharp pointed

white leaved

E. Indies h.h 5

spreading

Jamaica

S. Amer.

h.h $s$

downy

variegated

L. Indies

do.

h.h s

h.h s

DAJSY

garden

BARBERRY

common American

Europe

N.Amer. Med.\& h s

Diet

\section{BEET}

white

scarcity root or mangel zwurzel

common

BETONY

common or wood

\section{BIRCH}

white

Europe Med. h p

sweet-scented, or black

Europe Arts $\mathbf{h}$ t

horn-beam-lcaved

N. Amer. Arts h

$\begin{array}{llll}\text { do. Arts } & \text { h } & \text { t } \\ \text { do. Arts } & \text { h } & \text { t }\end{array}$ 


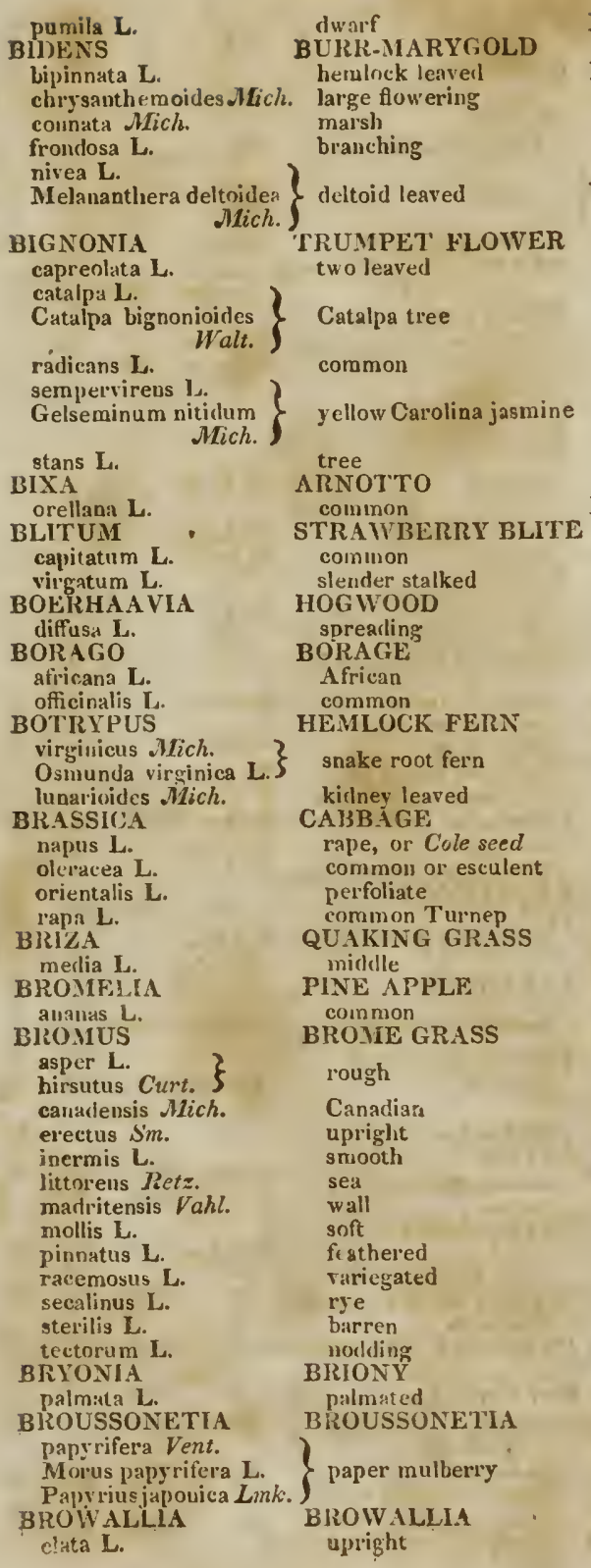

$\begin{array}{lll}\text { N. Amer. Aits } & \text { h } & \text { t } \\ \text { N. Amcr. } & \text { h } & \text { a } \\ \text { do. } & h & \text { a } \\ \text { lo. } & \text { h } & \text { a } \\ \text { do. } & \text { h } & \text { a }\end{array}$

W.Indies g.h p

N. Amer. g.h s

do. h t

do. h $t$

do. g.h s

S. Amer. li.h t

B. Indies Arts h.h s

Europe li a

do. h a

Peru h.h p

Africa li a

N. Amer. $\quad$ h p do. $h \quad p$

Europe Med. h p do. Diet h b do. Diet h b do. Dict h b

\section{Europe h b}

W.Indies Diet h.h $\mathrm{s}$

Europe h I

N. Amer. h

Europe $h$ p do. $h$ b

Siberia $h$ p

Europe h a

N.Anier. $\quad h$ a

Europe h p lo. h a

N. Amer. h a

Europe h a do. h a

F. Indies h.h p

Japan Arts h $t$

Pern h a 
HORTUS ELGINENSIS.

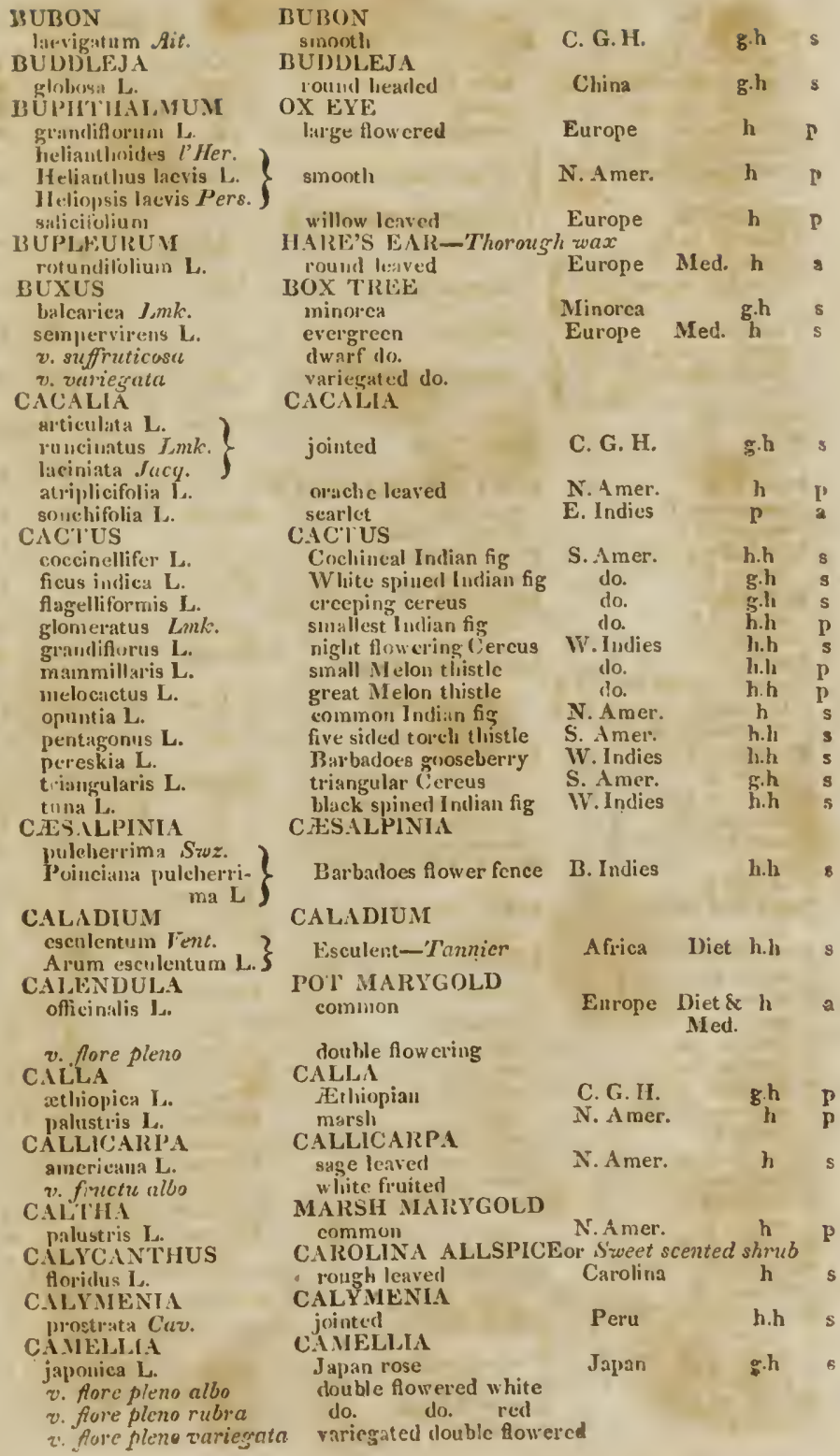




\section{CAMPANULA}

grancliflora $\mathbf{L}$. gentiannidles $L m k$. $\}$

lanuginosa $L m k$. merliuin L. perfoliata L. amplexicaulis $\mathrm{Nich}\}$ pyramidalis $\mathbf{L}$. rotundifolia L. speculum $\mathbf{L}$.

CANNA glauea l. v. faccida indica $L$. v. coccinea

CANNABIS sativa L.

CAPRIRIA biflora L.

CAPSICUM annuum L. ข. longum baceatum L. grossum $\mathrm{L}$.

\section{C.ARDAMINE}

impatiens $L$.

CARDIOSPER MUM halicacaluun $\mathrm{L}$.

\section{CARUUUS} arvensis $\operatorname{Sin}$.

Scriatula arvensis L.

Cnicus arvensis Pers.

defforatus $\mathrm{L}$. $\}$

cirsinides Vill. $\}$

horridulus Pers.

Cirsium horrilulum Nich.

lanceolatus $\mathrm{Sm}$.

$\left.\begin{array}{c}\text { Cnicus lanceolatus } \\ \text { Willdl. }\end{array}\right\}$

$\left.\begin{array}{l}\text { marianus L. } \\ \text { Carthamus maculatn: }\end{array}\right\}$ Lmk $\}$

Dersonata Willd.

CARICA

papaya L.

CARLINA

corymhosa L.

CARPINUS

Americana Willd.

CARTHAMUS

cerruleus L.

mitissimus $\mathbf{L}$.

tinctorius $\mathrm{L}$.

\section{CARUM}

carui L.

CASSIA

alata $\mathbf{L}$.

herpetica Jacq. $\}$

arborescens $V a h l$.

chamecrista L.

corvmbosa Lmk.

fusciculata Mich.

\section{BELL FLOWER}

large

woolly

Canterbury

perfoliate

pyramidal

round leared

Venus's Ionking-glass

INDI IN REED OI SHOT

glaucous

eommon

scarlct flowered

HEMP

common

CAPIRARIA

two flowered

CAPsicum or Guinea Pepper

common

long podied

Bird pepret

Bell pepper.

CARDAMINE

naked flow ering

HEART PEA

smooth leaved

THISTLE

Canata thistle

long leared

thoiny

lanceolate-mSpear thistle

dressed

P.IPAIV TREF

common

CAIRLINA

cluster flowering

HORN BEAM

A merican

CARTHAMUS or Safporver

blue

sniooth

bastard Saffron

CARAWAY

common

CASSIA

winged-Ring worm tree S. Amer. Med. h.h t

tree E. Indies

dwarf. Magothy bay bean N. A mer.

clustered

fasciculated
Europe

do.

Med.

h
g.h
h
h

g.h b

h $\quad$ p

g.h p

Art3 h $\quad$ a

h.h s

Diet h a

Med. g.h s

Diet g.h s

Europe

l1 a

li a

h b

h b

h b

h b

E. Indies Diet h.h

Italy g.h B

N. Amer. Arts h t

$\begin{array}{ccc}\text { Europe } & h & \mathbf{p} \\ \text { do. } & \mathbf{h} & \mathbf{p}\end{array}$

Egypt Mer.\& h Arts

Europe Med. h b

h h

h.h s

h 
nasilandica $\mathbf{L}$. nictitung $\mathbf{L}$. senna L. sophera $\mathbf{L}$. tora L. $\left.{ }_{\text {limensis } I, m k .}\right\}$

CASTANEA vesca Giert. Fagils castanea L. $\}$ pumila Wich. $\}$

CATANANCHE lutea $\mathbf{L}$.

C. TTFSBAL spinosa L.

CAUC ILIS maritanica $\mathbf{L}$

CEANOTHUS americanns $\mathbf{L}$.

CELASTRUS scantens L.

CELOSIA argentes L. coccinea $\mathbf{L}$. cristata L.

CELSIA

crectica L.

CELTIS

crassifolia Imnk.? cordata $H . P$. occiclentalis L.

CENCHRUS tribuloides L.

CEN'TAUREA

africana I $e s f$. atropurpurea Willd. benerlicta $\mathbf{L}$. centaureum L. cjocolylium $\mathbf{L}$. crupina L. cyanus $\mathbf{L}$. glauca Willd. mosclinta $L$. phrygia $\mathbf{L}$. scabiosa $\mathbf{L}$. solstitialis $\mathbf{L}$. spinosa $\mathbf{L}$ suaveoleus Willd? amberboi Imk. $\}$

CEPHALANTHUS meidenterlis $\mathbf{L}$.

CERAS'IIUM senidecandıum L. visensum $\mathbf{L}$. vilgatium l.s.

CERÁTONIA siliqua L. CEIRBERA thevetia L. CERCIS canadensis L. silicusstrum L. CFIRINTHE major $\mathbf{L}$. false Aoacia

shining or seusitive

Figytian senna

white

oval leaved

CHFSNUT

common

dwarf or chinquapin

CATANANCHE ycllow

LILY THORN

Bahama

BASE PARSLFY

small

NEW-JERSEY TEA-TRE

common

STAFF-TREF

climbing-Bitter-sweet

CELOSIA-Cock's comb

silvery spiked

scarlet spiked

commion

CELSIA

large flowered

NE'TLE TREE

dwarf

common American

CENCHRUS

hedge hog

CFN' $\Lambda$ URY

African

lark purple

Blessed thistle

great

blush flowered

black seeded

Blue Bottle

glaucous

Sweet Sultan

Austrian

scabinus

St. Barnaby's thistle thorny

yellow swcet Sultan

13U'T'TON WOOD

\section{American}

MOUSE EAR CHICIWEED

small

clanmy

commun

ST. JOHN'S BREAD

common

CERBERA or Ratle tree willow leaved

JUDAS TREE or Red bud

American

Europea

HONEY WORT

purple
N. Amer. Med. h do.

Egynt

E. Indics Med. h

S. Amer.

N. Amer. Diet \& h t A, ts

do. Diet h

Europe h a

W.Indies b.h s

Africa h

N. Amer. Med. h p

N. Amer. Med. h s

China

(io.

E. Indies

h a

Africa

g.h b

N. Amer. $\quad h \quad t$

do.

N. Amer. In a

\begin{tabular}{cccc} 
Africa & & g.h & b \\
Eurnpe & & h & p \\
do. & Med. & h & a \\
do. & & h & p \\
Syria & & h & a \\
Levant & & h & a \\
Europe & Med. & h & a \\
N. Amel. & & h & b \\
Peru & Med. & h & a \\
Europe & & h & p \\
do. & Med. & h & p \\
do. & & h & a \\
Crete & & g.h & s \\
\hline
\end{tabular}

Europe h a

N. Amer. Med. h s

N. Amer. h do. h a

Levant Med. g.h s

IV. Indies h.h t

N. Amer. Dict h t

Europe h t

Siberia 


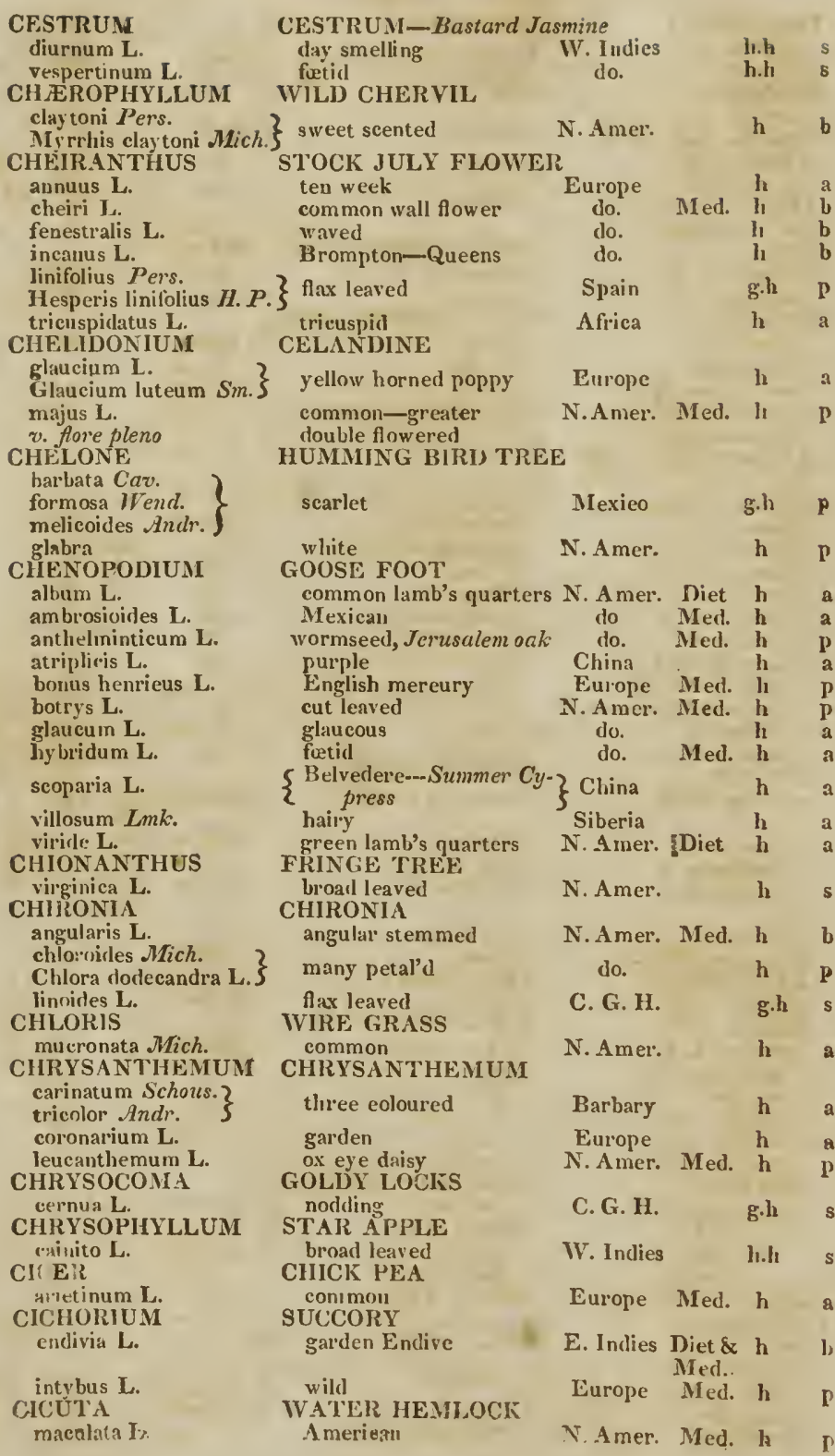


HORTLS RLGINENSIS.

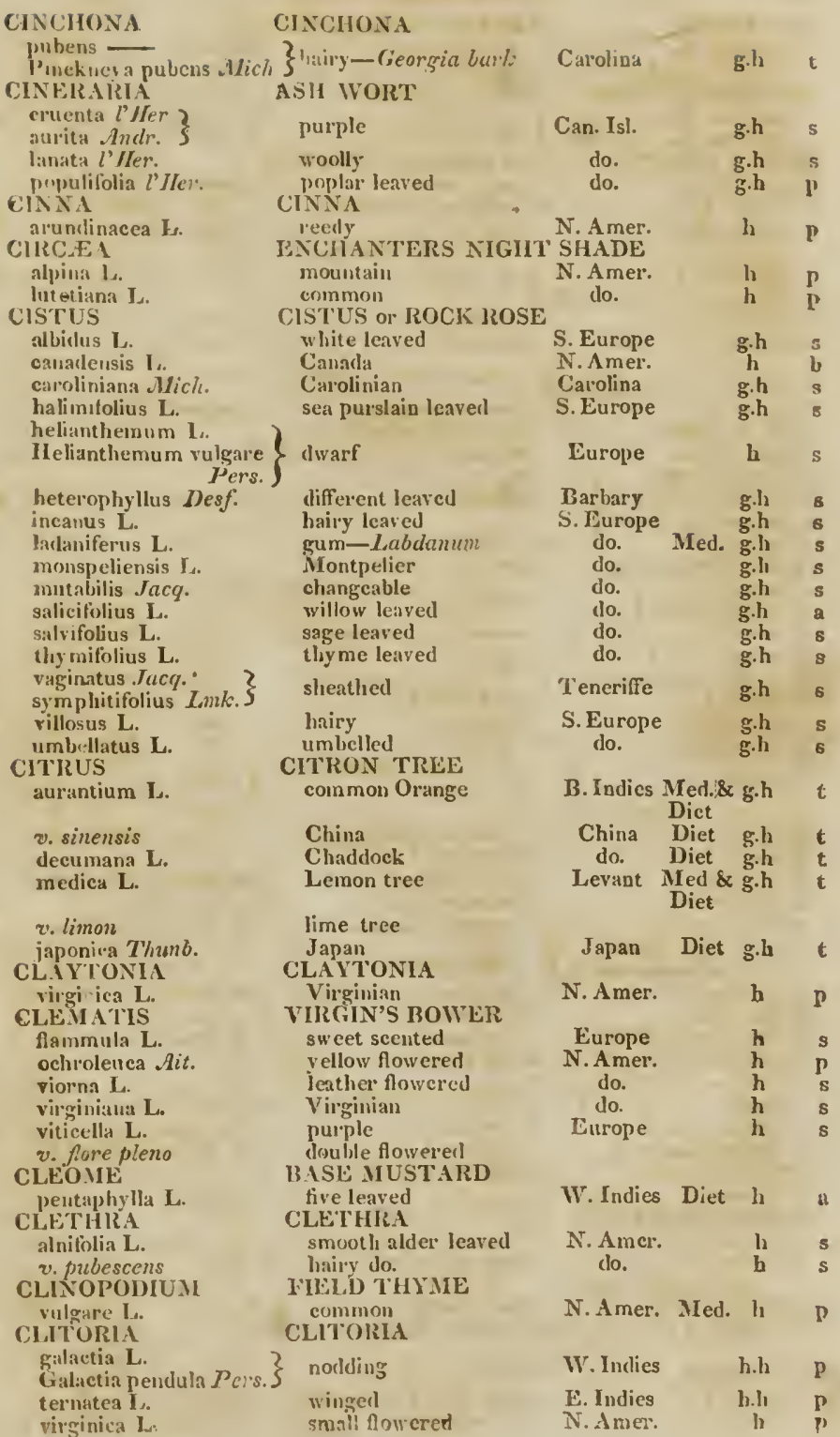


HORTUS ELGINENSIS.

\begin{tabular}{|c|c|c|c|c|}
\hline $\begin{array}{l}\text { CNEORUM } \\
\text { tricoccum L. }\end{array}$ & WIDOW WAIL & S. Euro & & g.h \\
\hline NICUS & $\begin{array}{l}\text { CNICUS-Thistle } \\
\text { fish }\end{array}$ & & & \\
\hline $\begin{array}{l}\text { casabonx Willd. } \\
\text { discolor Muhl. } \\
\text { oleraceus L. }\end{array}$ & $\begin{array}{l}\text { two coloured } \\
\text { yellow }\end{array}$ & $\begin{array}{l}\text { S. Eur } \\
\text { N. Am } \\
\text { Euro }\end{array}$ & & $h$ \\
\hline COCCOLOBA & $\begin{array}{l}\text { SEA SIDE GRAPE } \\
\text { small fruited }\end{array}$ & S. Amer. & & h.h \\
\hline $\begin{array}{l}\text { COCHLEARIA } \\
\text { armoracia } \mathrm{L} \text {. }\end{array}$ & $\begin{array}{l}\text { SCURVY GIRASS } \\
\text { horse radish }\end{array}$ & Europe & $\begin{array}{l}\text { Biet \& } \\
\text { Med. }\end{array}$ & i h \\
\hline officinalis $\mathbf{L}$. & common & do. & $\begin{array}{l}\text { Diet \& } \\
\text { Med }\end{array}$ & $\mathbf{~ h}$ \\
\hline OFFE & $\begin{array}{l}\text { COFFEE TREE } \\
\text { common }\end{array}$ & Arabia & $\begin{array}{l}\text { Diet \& } \\
\text { Med. }\end{array}$ & h.h \\
\hline OIX & $\begin{array}{l}\text { JOB'S TEARS } \\
\text { common }\end{array}$ & E. Indies & & g.h \\
\hline $\begin{array}{l}\text { SOLC } \\
\text { aute } \\
\text { v. } A\end{array}$ & $\begin{array}{l}\text { MEADOW SAFFRON } \\
\text { common } \\
\text { double flowered do. }\end{array}$ & Europe & Med. & h \\
\hline caLt & $\begin{array}{l}\text { COLLINSONIA-IIorse } \\
\text { common }\end{array}$ & $\begin{array}{l}\text { balm } \\
\text { N. Amer. }\end{array}$ & Med. & $\mathbf{h}$ \\
\hline & $\begin{array}{l}\text { BLADDER SENNA } \\
\text { common }\end{array}$ & Europe & & h \\
\hline $\begin{array}{l}\text { Ait. } \\
\text { lis } \mathbf{L m k}\}\end{array}$ & variegated & do. & & h \\
\hline L. & scarlet & C. G. H. & & g.h \\
\hline$k\}$. & pocock's & Levant & & g.h \\
\hline $\begin{array}{l}\text { COM } \\
\text { com } \\
\text { COM }\end{array}$ & $\begin{array}{l}\text { COMMELINA } \\
\text { common } \\
\text { COMPTONIA }\end{array}$ & S. Amer. & & g.h \\
\hline $\left.\begin{array}{c}\text { asplenifolia l'Her. } \\
\text { Liquidambar aspleni- } \\
\text { folium L. }\end{array}\right\}$ & - & N. Amer. & Med. & h \\
\hline $\begin{array}{l}\text { LARIA } \\
\text { L. } \\
\text { L. L. } \\
\text { ULUS }\end{array}$ & $\begin{array}{l}\text { MON'S SEAL } \\
\text { leaved or small } \\
\text { nou lily of the valley } \\
\text { flowered } \\
\text { non } \\
\text { ererl } \\
\text { WEED }\end{array}$ & $\begin{array}{l}\text { N. Amer. } \\
\text { Lurope } \\
\text { N. Amer. } \\
\text { do. } \\
\text { do. }\end{array}$ & $\begin{array}{l}\text { Med. } \\
\text { Med. } \\
\text { Med. } \\
\text { Med. }\end{array}$ & $\begin{array}{l}\text { h } \\
\text { h } \\
\text { h } \\
\text { h } \\
\text { h }\end{array}$ \\
\hline & $\begin{array}{l}\text { WEED } \\
\text { ous-szueet potatoe }\end{array}$ & B. Indies & Diet & $\mathbf{h}$ \\
\hline $\begin{array}{l}\text { L. } \\
\text { Mich. }\end{array}$ & $\begin{array}{l}\text { mealy } \\
\text { fiddle leaved will potatoe } \\
\text { arrow leaved }\end{array}$ & $\begin{array}{l}\text { Madleira } \\
\text { N. Amer. } \\
\text { do. }\end{array}$ & Med. & $\begin{array}{c}\text { g.h } \\
h \\
h\end{array}$ \\
\hline & & & & \\
\hline & $\begin{array}{l}\text { eaved } \\
\text { loured } \\
\text { li }\end{array}$ & $\begin{array}{l}\text { Europe } \\
\text { do. } \\
\text { w. Indies }\end{array}$ & d. & $\underset{h}{h}$ \\
\hline & BANF: & & & \\
\hline $\begin{array}{l}\text { illd. } \\
\text { fetidum L } \\
\text { ca Mich. }\end{array}$ & foetid & Africa & & g.h \\
\hline Erigeron camp & marsh & N. A met. & & h \\
\hline & $\begin{array}{l}\text { pleaved } \\
\text { BA BALSAM TR }\end{array}$ & S. 1 & & $\mathrm{~g}$ \\
\hline & iUS & S. Amer. & Med. 1 & h. $l_{1}$ \\
\hline & $\begin{array}{l}\text { bristly leaved } \\
\text { germander learer }\end{array}$ & $\begin{array}{l}\text { B. Indies } \\
\text { do. }\end{array}$ & & \\
\hline
\end{tabular}




\begin{tabular}{|c|c|c|c|c|c|}
\hline & $\begin{array}{l}\text { CORDIA } \\
\text { yellnw fruterl }\end{array}$ & S. Amer. & & h.h & \\
\hline & TICK-SEEJ SUN-FLOW & VER & & & \\
\hline & $\begin{array}{l}\text { narrow leared } \\
\text { ear leaved }\end{array}$ & $\begin{array}{l}\text { N. A mer. } \\
\text { do. }\end{array}$ & & $\begin{array}{l}\mathbf{h} \\
\mathbf{h}\end{array}$ & $p$ \\
\hline & $\begin{array}{l}\text { three leaved } \\
\text { whorl leaved }\end{array}$ & $\begin{array}{l}\text { do. } \\
\text { do. }\end{array}$ & & h & \\
\hline CORI I NIDIRUM & CORIANDER & & & & \\
\hline $\begin{array}{l}\text { satiun } \\
\text { co!rNUS }\end{array}$ & $\begin{array}{c}\text { common } \\
\text { DOGIVOOD }\end{array}$ & Europe & Met. & $\mathbf{h}$ & \\
\hline $\begin{array}{l}\text { CO!RNUS } \\
\text { alba L. }\end{array}$ & white herried & N. Amer. & & h & $\$$ \\
\hline $\begin{array}{l}\text { alternifolia L. } \\
\text { canarlensis L. }\end{array}$ & $\begin{array}{l}\text { alternate leaved } \\
\text { Canadian }\end{array}$ & $\begin{array}{l}\text { do. } \\
\text { do. }\end{array}$ & & $\begin{array}{l}\mathrm{h}_{\mathrm{h}} \\
\mathrm{h}_{\mathrm{h}}\end{array}$ & \\
\hline circinata l'Her. & bairy & do. & & h & \\
\hline $\begin{array}{l}\text { tomentulosa. } \\
\text { florida } \mathbf{L} \text {. }\end{array}$ & eommon & & Med. & h & $t$ \\
\hline mascula L. & an Cherry & Europe & & & \\
\hline $\begin{array}{l}\text { paniculata } \mathbf{L} \text {. } \\
\text { sanguinea } \mathbf{L} \text {. }\end{array}$ & $\begin{array}{l}\text { paniculated } \\
\text { bloody }\end{array}$ & $\begin{array}{l}\text { N. Anier. } \\
\text { do. }\end{array}$ & & $\begin{array}{l}\mathbf{h} \\
\mathbf{h}\end{array}$ & \\
\hline 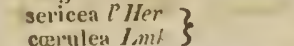 & blue berried & do. & & h & $s$ \\
\hline $\begin{array}{l}\text { stolonifera. Jich. } \\
\text { striet a l'Ifer. }\end{array}$ & $\begin{array}{l}\text { creeping } \\
\text { upriglit }\end{array}$ & $\begin{array}{l}\text { do. } \\
\text { do. }\end{array}$ & & $\begin{array}{l}\mathbf{h} \\
\mathbf{h}\end{array}$ & s \\
\hline CORONILLA & CORONILLA & & & & \\
\hline $\begin{array}{l}\text { aculeatia } 11 \text { illd. } \\
\text { Seshonia aculeata } 7 \text { ers. }\end{array}$ & prickly & E. Indies & & h & $\mathfrak{a}$ \\
\hline $\left.\begin{array}{l}\text { argentea L. } \\
\text { stipularis L.mk }\end{array}\right\}$ & silvery & Crete & & g.h & s \\
\hline $\left.\begin{array}{l}\text { eamabisa IIilld. } \\
\text { Sesbania cannabin. } \\
\text { Pers }\end{array}\right\}$ & hemp & E. Indies & & $\mathrm{h}$ & a \\
\hline $\left.\begin{array}{l}\text { corcinea Hilld. } \\
\text { IEscliynomene cocci- } \\
\text { nea L. }\end{array}\right\}$ & scarlet flowered & do. & & h.h & 3 \\
\hline $\begin{array}{l}\text { Sesbania coccineaPers. } \\
\text { emerus L. } \\
\text { glauca L. }\end{array}$ & $\begin{array}{l}\text { seorpion senns } \\
\text { seven leaved }\end{array}$ & $\begin{array}{l}\text { Europe } \\
\text { S. France }\end{array}$ & & $\begin{array}{c}\text { h } \\
\text { g.h }\end{array}$ & $\begin{array}{l}\text { s } \\
\text { s }\end{array}$ \\
\hline $\left.\begin{array}{r}\text { gr:andifiora Willd. } \\
\text { Eschynomene gran. } \\
\text { diffor'a L. L. }\end{array}\right\}$ & large flowered & F. Indies & & h.h & $\mathbf{s}$ \\
\hline $\begin{array}{l}\text { varia } L \\
\text { CoryLUS }\end{array}$ & $\begin{array}{l}\text { various coloured } \\
\text { HAZEL-NUT or Filbert }\end{array}$ & Europe & & $\mathrm{h}$ & $\mathrm{p}$ \\
\hline $\begin{array}{l}\text { americana Mhich. } \\
\text { avellana } \mathbf{L} \text {. }\end{array}$ & $\begin{array}{l}\text { A merican common } \\
\text { Furolieall common }\end{array}$ & $\begin{array}{l}\text { N. Amer. } \\
\text { Europe }\end{array}$ & $\begin{array}{l}\text { Diet } \\
\text { Diet \& } \\
\text { Arts }\end{array}$ & $\begin{array}{l}\mathrm{h} \\
\mathrm{h}\end{array}$ & $\begin{array}{l}t \\
t\end{array}$ \\
\hline $\begin{array}{l}\text { rostrata Ait. } \\
\text { tuluulosa } \mathbf{W i l l d .}\}\end{array}$ & euckold or beaked & N. Amer. & Diet & h & $t$ \\
\hline $\begin{array}{c}\text { tulsulosa } \\
\text { maxina } L m k . \\
\text { Cos'rus }\end{array}$ & $\begin{array}{l}\text { large Spanish } \\
\text { cosTus }\end{array}$ & Europe & Diet & h & $\mathbf{t}$ \\
\hline $\begin{array}{l}\text { speciosus Sm. } \\
\text { aralicus Jacq. } \\
\text { A nomum hissutum } \\
\text { Lmk. }\end{array}$ & large flowering & E. Indies & & h.h & $p$ \\
\hline $\begin{array}{l}\text { COTYELDON } \\
\text { hremisplerica L. } \\
\text { CR UIBE }\end{array}$ & $\begin{array}{l}\text { 1. WORT } \\
\text { leaved } \\
\text { or COLE WORT }\end{array}$ & C. G. H. & & g.h & s \\
\hline $\begin{array}{l}\text { CR.IIBBE } \\
\text { minitima L. }\end{array}$ & $\begin{array}{l}\text { A.A.E Or COLE } \\
\text { sea }\end{array}$ & Europe & Diet & $\mathbf{h}$ & p \\
\hline $\begin{array}{l}\text { CRATHETUS } \\
\text { eoccinea L. } \\
\text { cordata Ait. }\end{array}$ & $\begin{array}{l}\text { II.IW-THORN } \\
\text { scarlet fruited }\end{array}$ & N. Amer. & Arts & h & $\mathbf{s}$ \\
\hline $\begin{array}{l}\text { a H.P. } \\
\text { us accrifoliaPoir. }\end{array}$ & & do. & & h & s \\
\hline $\begin{array}{l}\text { crus galli L. } \\
\text { oryacant ha I. }\end{array}$ & $\begin{array}{l}\text { cock spur } \\
\text { Mnımon white }\end{array}$ & $\begin{array}{l}\text { do. } \\
\text { Eurone }\end{array}$ & Arts & $\begin{array}{l}h \\
h\end{array}$ & i \\
\hline
\end{tabular}




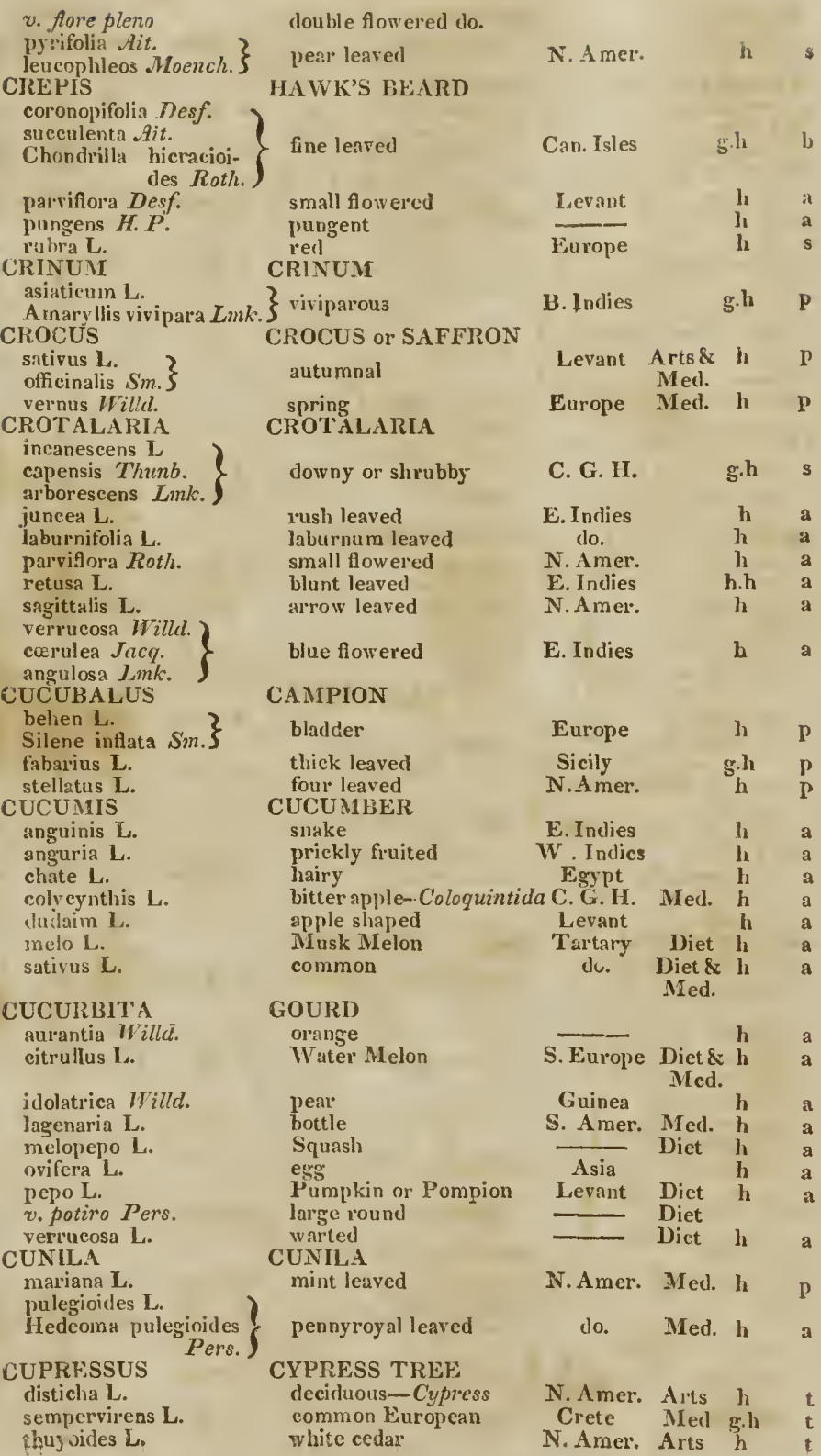




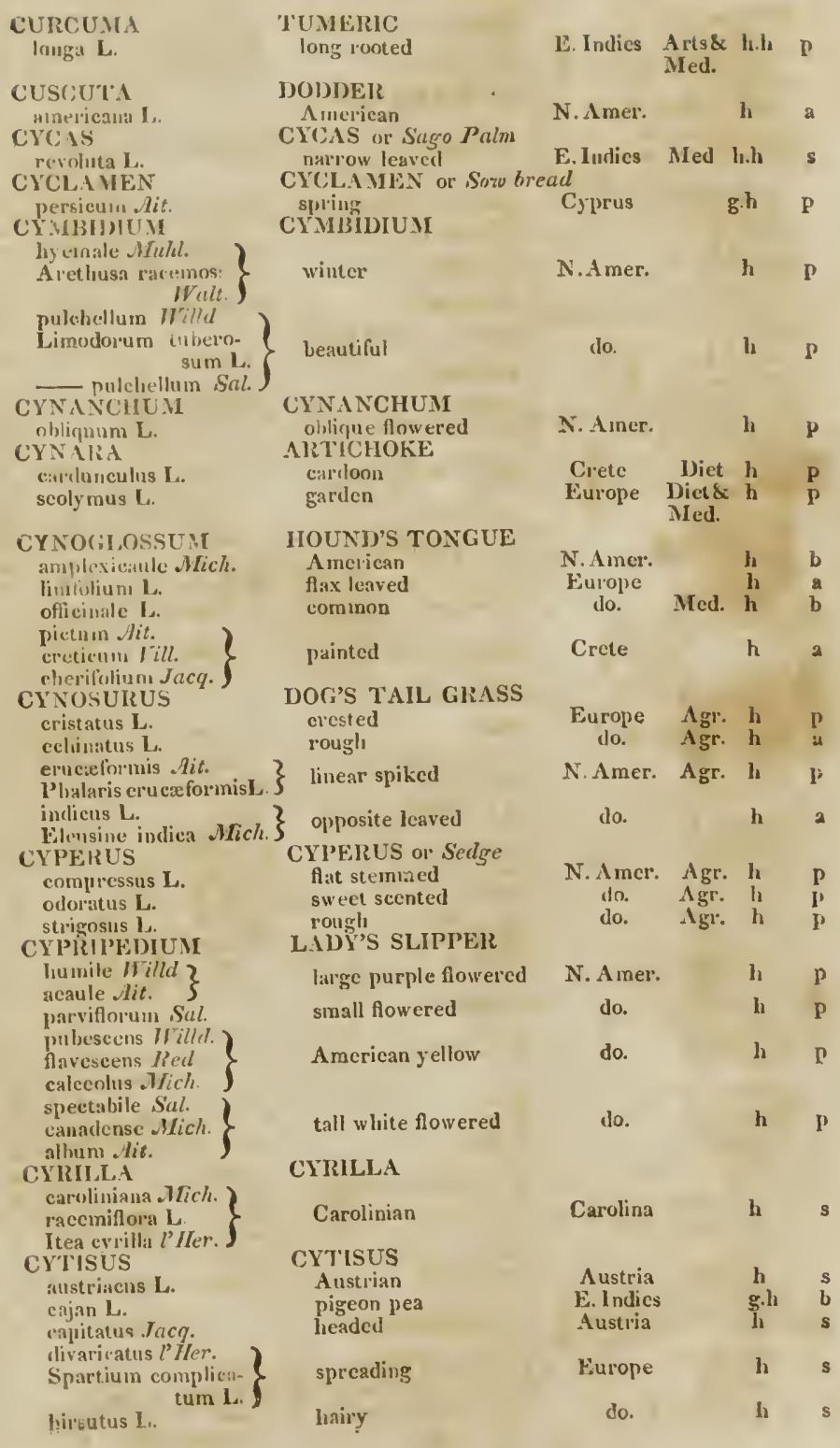


HORTUS ELGINENSIS.

laburnumL.

nigricans $\mathbf{L}$.

se-ssibifolius L.

supinus $\mathbf{L}$.

DAC'TYLIS

glomerata L.

DALEA

alopecurnides Willd

limmi Mich.

DAPHNE

mezereum $\mathbf{L}$.

olora Ait.

tartouraira $\mathbf{L .}$

DA TURA

ferox L.

lavis $\mathbf{L}$.

metel L.

stramonium $\mathbf{L}$.

tatula L.

DAUCUS

carota

labur'num

Austria

blackish

clamny podded

trailing

COCK'S FOOT GRASS

Europe

Italy

5 round hearled-OOr?

Europe

1) ILEA

N.Amer. Agr. h p

fox tail or silky spiked N.A mer.

ha

DAPINE

common Mezereon

Eirrope Med. h

swect scented

China

silvery

THORN APPLE

S. Europe

long spined

smionth

China

downy

A frica

do.

cominon-Jameston rueed N.Amer. Med. h

blue

do. Med. $h$

CARROT

garden

Europe Diet \& h

wild

v. sulvestris

DELIPHINIUM

ajacis $\mathbf{L}$.

consulida $L$.

LARK SPUR

rocket

field

$\left.\begin{array}{l}\text { hybridum Willd. } \\ \text { hirsutum Pers. }\end{array}\right\} \quad$ hairy

staphisagria $\mathbf{L}$.

DESMANTHUS Filld
MIMOSA Azct.

stavesacre

Med.

N. Amer. Med. h

Europe do.

Med. $\stackrel{h}{h}$

Siberia

Europe Med. h

cinereus Willd.

virgatus II illd.

DIAN'THUS

atrorubens $\mathrm{Jacq}$.

barbatus $\mathbf{L}$.

carthusianorum L.

caryophyllus $\mathbf{L}$.

cbinensis $\mathrm{L}$.

collinus Wald.

deltoides $\mathbf{L}$.

plumarius $\mathbf{L}$.

supertus $\mathrm{L}$.

DICHONDIRA

sericen Suz.

DIC'TAMNUS

albus $\mathbf{L}$.

DIGITALIS

lutea $\mathbf{L}$.

purpinrea L.

ข. alba

DION $B \mathrm{AA}$

muscipula $\mathbf{I}_{\text {. }}$.

DIOSCUREA

alata $\mathbf{L}$.

sativa $\mathbf{L}$

villosa $\mathbf{L}$. panieulata . Mich. $\}$

DIOS.MA

trimirles L.

DIOSI'YROS

virginiana L.

\}DESMANTHUS

grey

slender twigged

PINK

purplish

Sweet William

Cartlusian

Carnation or garden

Chinese

Hungarian

maiclen

feathered

night snented or fringed

DICHONDRA

silvery

FRAXINELLA

cominon

DIGITALIS or Fox-glove yellow

puiple or common

white

VENUS'S FLY TRAP sensitive

YAM

winged stem'd

cultivat ed

$\Lambda$ merican

DIOSMA

heath leaved

PERSIMON or Date Plamb common-Medlar
E. Indies

clo.

Europe

$$
\text { do. }
$$

do.

Europe

China

Hungal'y

Europe

do.

do.

W. Indies

Europe

Europe do.

h.h s

fi.h s

Med. $\begin{array}{cc}\mathbf{h} & \mathbf{p} \\ \mathbf{h} & \mathbf{p} \\ \mathbf{h} & \mathbf{p} \\ \mathbf{h} & \mathbf{p} \\ \mathbf{h} & \mathbf{p} \\ \mathbf{h} & \mathbf{p} \\ \mathbf{h} & \mathbf{p} \\ \mathbf{h} & \mathrm{b}\end{array}$

h.h $\mathrm{p}$

Med. h p

do. Med. h

Carolina g.h

E. Indies Dict h.h

1o. Diet h. P

N.Amer. $\quad h \quad p$

C. G. H.

g.h s

N.Amer. Diet \& h t 


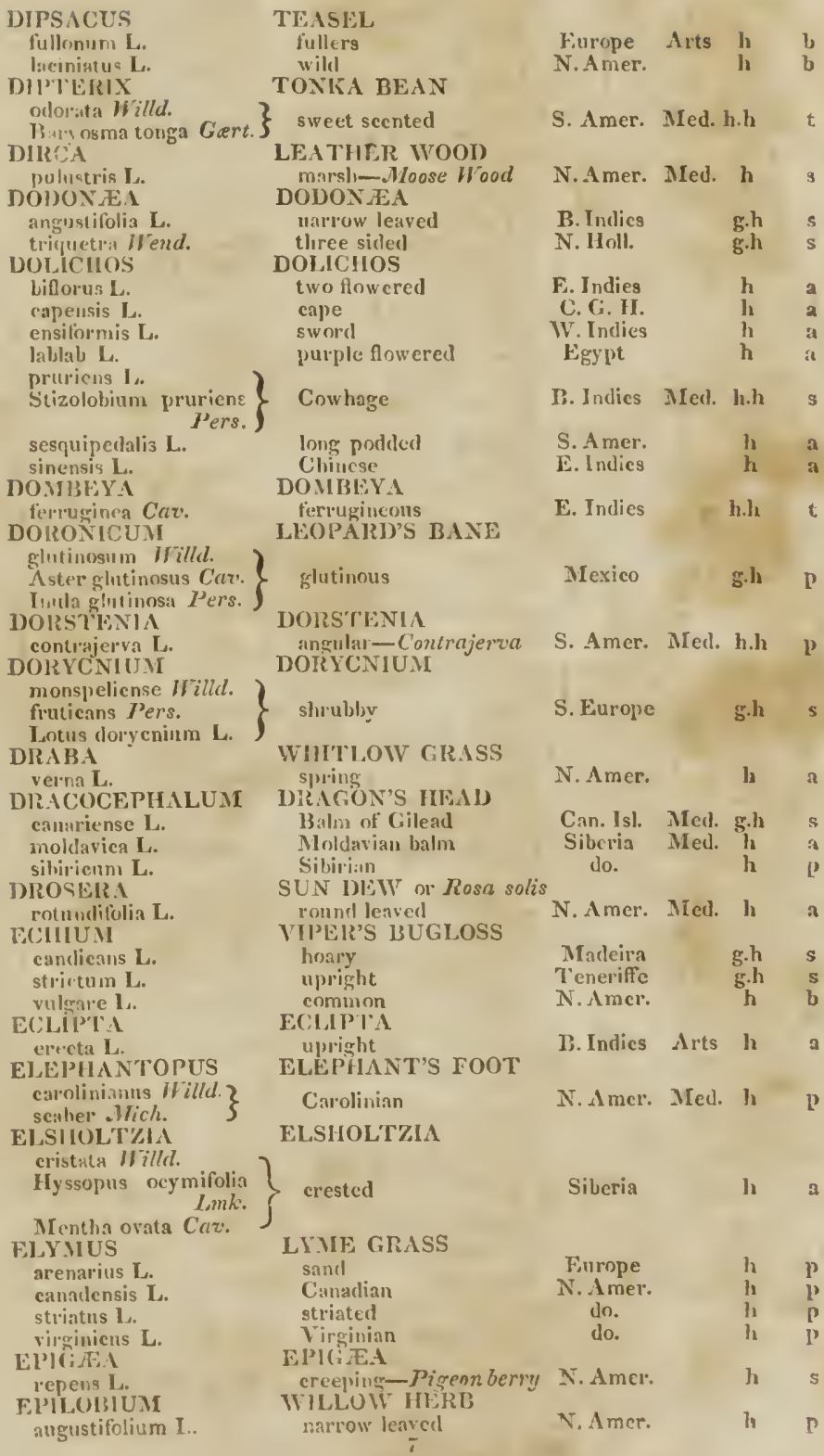


HORTUS ELGINENSIS.

spicatum $L m k$. pubescens $R$ oth.

villusum Ait. molle Lmk.

tetragonum $\mathbf{L}$.

ARICA

arboren L.

australis $\mathbf{L}$.

herbacea $L$.

mediterranea $\mathbf{L}$.

scopsiria L.

tetralix L.

stricta Donn.

trifion'a L.

tubiflora L.

vagans $\mathrm{L}$.

vulgaris $\mathbf{L}$.

v. flore pleno

ERIGERON

canadense $\mathbf{L}$.

heterophyllum $M$ Iuhl

pliiladelphicum $\mathbf{L}$.

strigosum Muhl.

ERIOPHORUM

cyperinum L.

Scirpus eriophorum

Trichophorum .Vich. rinum Pers.

polystachyon I.

ERODIUN $\mathrm{lit}$.

GERANIUM Auct. $\}$

ciconium Irilld.

cicutarium $\mathrm{S}$.

moschatum Ait.

ER V U

lens L.

tetraspermum L.

FIYNGIUM

aquaticum $\mathbf{L}$.

dilataturn $L m k$.

planum L.

suaveolens Brors.

yuccifolium Mich.

PRYSIMUM

barbarea L.

officinale $\mathrm{L}$.

EIVTIIRLNA

corallodendron L.

crista galli L. 3

laurifolia Jacq. $\}$

herlacea 1.

ERYIHRONIUM

leus canis $L$.

EUCALYPTUS

robusta $S \mathrm{~m}$.

EUCOMIS

punctata l'Her.

F.UGENIA

jambos L.

unilora

FUONYMUS

americanus $I$.

narrow leaved

N. $\mathbf{A}$ mel.

h

soft leaved

Europe

N. Amer.

h p

square stalked

l) p

HE. I TII

tree

Spauish

carly dwarf

Mediterranean

small flowering

cross leaved

uplight

thiee flowered

tubular

Cornish

common

Marleira

S. Europe

do.

do.

do.

Europe

Corsica

C. G. H.

do.

Europe

do.

g.h 5

g.h s

g.h s

g.h 5

h s

g.h s

g.l s

g.h s

g.h s

g.h s

ERIGEIRON or Flea Bane

common fieis

difielent leaved

spreading

liarsh

N. Amel. Ned.

do.

do.

do.

CO'TON GRASS-Wool grass.

tall

N. A mer.

h a

h a

b a

common

do.

h

IIERON'S BILL, GERANIUM

hairy

hemlock lcaver

musk smelling

S. Europe

Europe

do.

TARE

common

four seetled

ERY NGO

water

broad leaved

flat leaved

sweet scented

vucea leavel

HEDGE MUSTARD

winter cress

comnon

Europe Med. h a do.

N. Amer. Med. h p

Europe $h$ p do.

S. Europe

N. Amer.

h

Furope Med. l,

N. Amel. Med. li

COLA], TREE:

common

S. Amer.

l.h

cockscomb

do.

h.h $s$

herbaceous

DOG'S TOOTH VIOLET common

Carolina Med. g.h p

EUC.ILYPTUS

ural leaveal

CHOWN ASPHODEL spotterl

EUGFNIA

N. Amer: h

S Malabar plumb-Pome

$\{$ rose

Indian cherry tree

N. Holl.

g.h

C. G. II.

g.h p

E. Indies Med.\& $\mathrm{g} . \mathbf{h}$ s

SPINI)LE T'RE

A.merican

S. Amer. Diet g.h

N. $\Lambda \mathrm{mel}$.

h s 
europseus $\mathrm{L}$.

EU'\&TORIUM

ageratoides Willd.

urticifolium Willd.

arnmaticum L.

coronopifolium Willd.

Churysucoma rorounpi folia Mich.

foniculacenm IJilld.

Chrysocoma capillace:

maculatum I.

perfoliatum L.

рuрureum L.

sessilitolium L.

tencrifolium Willd.

trifoliatunı L.

verticill:tum Mulil.

BITIIORIBIA

chamesyec. $L_{\text {. }}$.

cha:acias 1.

cvparis ias L.

hypesicitolia $\mathbf{L}$.

lathyris $\mathbf{L}$.

maculata L.

jeplus L.

polygonifolia L.

punicea Jacq.

spinos: $\mathbf{L}$.

verucosa $L$.

FAGUS

ferruginea Ait. ?

svlvestris Pers.

FERRARIA

$\left.\begin{array}{l}\text { maculata L. } \\ \text { punctata Pers. }\end{array}\right\}$

FESTUCA

bromoides

elatior L.

fluitans 1.

loliacea Sm.

elougata Ehrt.

myurus L.

ovina L.

poxeformis Pers.

rubra $\mathbf{L}$.

spadicea L.

tenuifolia Leers.

\section{FiCUS}

carica $\mathbf{L}$.

stipulata Thunb.

scaurlens Imk.

FOTIERCiLLA

aluifolia $\mathbf{L}$.

FR.AG.ARIA

chiloensis Ehrt.

elatior F/hrt.

granliflora Ehrt.

vesea 1.

r. alpina

virginiana Ehrt.
Filupean

EU'A TORIUM

nettle leaved

aromatic

coronopus lcaved

dog's fennel

sported stalked

bone-sett-IYlorough-wort

purple stalkerl

sessile leaverl

germander leaved

three leaved

whomled

SPURGE

small

cypress

willow leaved

St. John's wort leaved

caper

spotted

petty

polygonum leaved

scailet

thorny

warted

BEACH

common American

FEIRARIA

spotted

\section{FESCUE GRASS}

brome

tall meadow

flouting:

long spiked

one spiked

wall

sheeps

spreading

meadow

sheathed

fine leaved

FIG TREE

common

crecping

FOTHERGILLA

alder leswed

STR. IWBERRY

Cliili

hautloy

pinc-apple

counmon garden

monthly

wild
Europe Arts h s

N. Ainer. h $\mathrm{r}$

do.

do.

$$
\text { h }
$$

p

Jo.

p

do.

do.

do.

do.

do.

clo.

do.

Europe

clo.

S. Eurnpe

IV. Iudies

h a

N. Arner.

do.

do.

W. Indies

S. Europe

do.

Med. $\begin{array}{ll}\mathbf{h} & \mathbf{p} \\ \mathbf{h} & \mathbf{p}\end{array}$

h

h

h

p

p

p

h

Med. h

$a$
$p$
$s$
$a$
$b$
$a$
$a$
$a$
s
s
$b$

N. Amer. Arts h t

C. G. I.

g.h

Europe Agr. h

do. Agr.

do. Agr.

do. Agr. h

Barbary

Europe Agr.

do. Agi. h

do. Agr. h

do. A giv. h

do. $A$ r.

do. Agr. h

S.Europe Med.\& g.h t

Diet

E. Indies Diet h.h s

N. Amer. H

S. Amer. Diet h p

ilo. Jiet h p

do. Dict h p

Europe l)iet h p

N. Amer. Diet $h$ p 


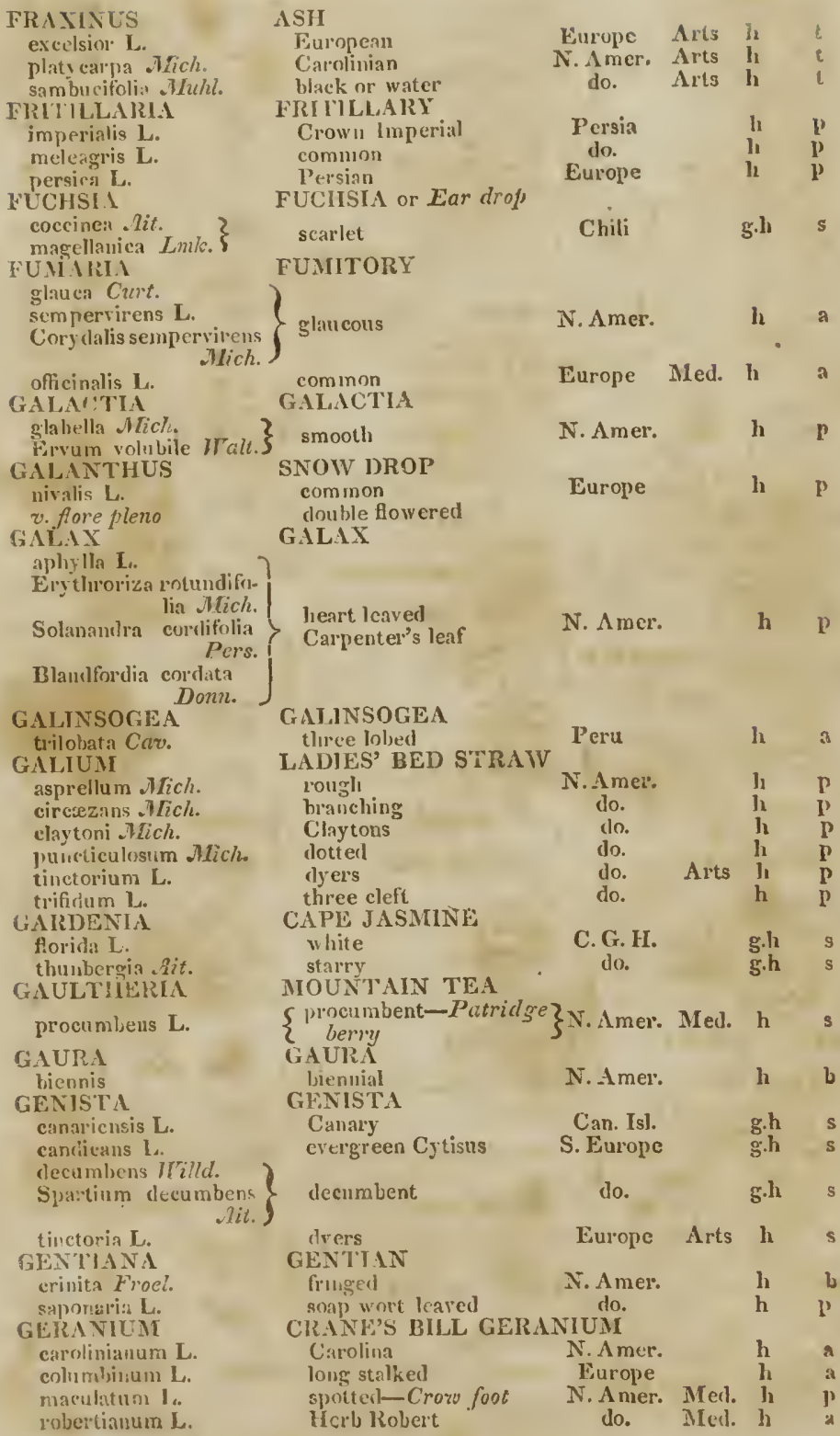


HORTUS ELGINENSIS.

\section{sanguineum $\mathbf{L}$, v. prostratum sylvaticum $\mathbf{L}$. \\ GFIR ARDIA \\ crinita - \\ กิเขล $\mathbf{L}$. \\ glauca \\ perlicularia $\mathbf{l}$. \\ purpurea $\mathbf{L}$. \\ tenuifolia Vahl.? \\ erceta Walt. $\}$}

\section{GEUM}

rivale $\mathbf{L}$.

strictum Ait.
aleppicum Iacq.

virginiarum $\mathbf{L}$.

GLAIJOLUS

earneus Jucq.

conmunis L.

sccuriger Ait.

trist is $\mathbf{L}$.

GLECHOMA

hederacea $L$.

CLEDITSIA

sinensis $\operatorname{Lmk}$.

triacanthos L.

v. inermis

GLORIOSA

Nuperhonica superba $\boldsymbol{H} . \boldsymbol{P}$. $\}$ limibing

bloody.

creeping

wood

GERARDIA

fringed

yellow

glaucous

louse wort leared

purple

finc leaved

AVENS or Merb Bennet

water

upright

virginianum

CORN FLAG

flesh coloured

conmmon

copper coloured

square stalked

GROUND IVY

common

CILDITSIA

Chinese

three thorned Acacia smooth do.

SUYERB LILY

\section{GLYCINE}

angulosa $\boldsymbol{M} i h l$.

apios $\mathbf{L}$.

bimsculata Curt.

$\left.\begin{array}{r}\text { Kennedia monophyll. } \\ \text { Pers. }\end{array}\right\}$

caribra Jacq.

coccinea Curt.

Kennedia coceine:

GLYCINE

angular

tuberous

comosa L.

frutescens $\mathbf{L}$.

monoica $\mathbf{L}$.

$$
\text { Pers. }
$$

simple leaved

hairy podded

scarlet flowered

hairy

shrublyy

monecious

$\left.\begin{array}{l}\text { rubicunda } \text { Curt. } \\ \text { Kennedia rubicuml. }\end{array}\right\}$ red flowered

\section{GLYCYRRHIZA} glabra L.

\section{GNAPHALIUM}

margaritaceum $\mathbf{L}$ olstusifolium $\mathbf{L}$. $\left.\begin{array}{l}\text { polycephalum Mich } \\ \text { conoideum Lmk. }\end{array}\right\}$ plantaginifolium L. purpureum L. stcechas 1.

uliginosum 1. .

GOMPHIRENA globosa $\mathbf{L}$.

ROODENIA Pers. LIQUORICE

common CUDWEED-Life Everlasting
N.Am

obtusc leaved

plantain leaved

purple

narrow leared

marsh

GLOBF: AMARANTH

garden GOODENIA

lavigata Curt.
Sexvola levigata Pers, smouth

$\begin{array}{ccc}\text { Europe } & \text { h } & \text { p } \\ \text { do. } & \text { h } & \text { p } \\ \text { N. Amer. } & \text { h } & \text { a } \\ \text { do. } & \text { h } & \text { p } \\ \text { do. } & \text { h } & \text { p } \\ \text { do. } & \text { h } & \text { p } \\ \text { do. } & \text { h } & \mathbf{a} \\ \text { do. } & \text { h } & \mathbf{a}\end{array}$

N. Amer. Med. h p do. Med. li $p$ do. Is $p$

C. G. H.

S. Hurope

C. G. H.

do.

$\begin{array}{cc}\text { g.h } & \text { p } \\ \text { h } & \text { p } \\ \text { g.h } & \text { p } \\ \text { g.h } & \text { p }\end{array}$

N.Amer. Med. h p

China Arts g.h t. N. Aner. Arts is $t$

E. Indies h.h p

N. Amer. li a do.

h r

N. Holl.

g.h $\$$

W. Indies

h.h s

N. Holl.

g.h s

N. Amer.

ro.

clo.

h a

h s

N. Holl. $\quad$.h $\mathrm{g}$

Furnpe Med. h p N.dmer. h p

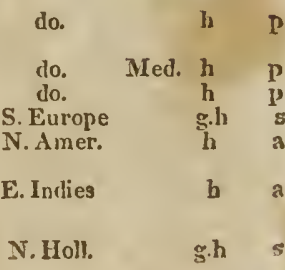




\begin{tabular}{|c|c|c|c|c|c|}
\hline GORDONIA & GORDONIA & & & & \\
\hline $\left.\begin{array}{c}\text { Franklinia alatamaha } \\
\text { Bartr. }\end{array}\right\}$ & Fi'ankliu's & N. Amer. & & $\mathrm{I}_{2}$ & $\mathbf{s}$ \\
\hline lasianthus $\mathbf{L}$. & loblolly bay & do. & & h & $\mathbf{s}$ \\
\hline GOSSYPIUM & COTTON & & & & \\
\hline $\begin{array}{l}\text { herbiceum } \mathbf{L} \text {. } \\
\text { religiosum } \mathrm{L} \text {. }\end{array}$ & $\begin{array}{l}\text { common } \\
\text { Nankin }\end{array}$ & $\begin{array}{l}\text { B. Indies } \\
\text { E. Indies }\end{array}$ & $\begin{array}{l}\text { Arts } \\
\text { Aits }\end{array}$ & $\begin{array}{l}h \\
\text { h.h }\end{array}$ & $\mathbf{a}$ \\
\hline GRATIOLA & HEWGE HYSSOP & & & & \\
\hline amagallidea NIch. & chickweed leaved & N. Amer. & & h & a \\
\hline $\begin{array}{l}\text { oficinalis L. } \\
\text { v. caroliniensis Pers. }\end{array}$ & common & & & h & a \\
\hline GRE IVIA & GREIVIA & & & & \\
\hline $\begin{array}{l}\text { occientalis L. } \\
\text { orientalis L. }\end{array}$ & eastern & E. Indies & & h.h & $\mathrm{t}$ \\
\hline GUETTARDA & GUEITARDA & E Indies & & ho & $t$ \\
\hline $\begin{array}{l}\text { speciosa L. } \\
\text { GUILANDINA }\end{array}$ & $\begin{array}{l}\text { shewy } \\
\text { BONDUC or Nicker tree }\end{array}$ & $e^{\text {L. Intiles }}$ & & & 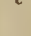 \\
\hline $\begin{array}{l}\text { bonduc L } \\
\text { GYMNOCLADUS }\end{array}$ & $\begin{array}{l}\text { yellow seedler } \\
\text { GYMNOCLADUS }\end{array}$ & E. Indies & Diet & h.h & $\mathbf{t}$ \\
\hline cauadensis Nich. $\}$ & Kentucky coffee tree & N. Amer. & Diet & $\mathbf{h}$ & $t$ \\
\hline GXPSOPHYLLA & GYPSOPHYLLA & & & & \\
\hline $\begin{array}{l}\text { perfoliata } \mathbf{L} . \\
\text { viscosit } \mathbf{L} \text {. }\end{array}$ & $\begin{array}{l}\text { perfoliate } \\
\text { clammy }\end{array}$ & $\begin{array}{c}\text { Europe } \\
\text { (lo. }\end{array}$ & & $\begin{array}{l}\mathrm{h} \\
\mathrm{h}\end{array}$ & $\begin{array}{l}\mathrm{p} \\
\mathrm{a}\end{array}$ \\
\hline HALESIA & SNOW DIROP TREE OI & Silver Bel & & & \\
\hline tera L. & fou & Carolina & & h & s \\
\hline $\begin{array}{l}\text { HALORAGIS } \\
\text { cercodia dit. }\end{array}$ & HALORAGIS & & & & \\
\hline $\begin{array}{l}\text { tetragouia l'IIer. } \\
\text { alata Jucq. } \\
\text { Tetragonia ivæ folia L. }\end{array}$ & $\begin{array}{l}\text { whorl leaved } \\
\text { wing seeded }\end{array}$ & S. Sea Isl. & & g.h & $\mathbf{s}$ \\
\hline $\begin{array}{l}\text { HAMAMELIS } \\
\text { virginica L. }\end{array}$ & WITCH HAZEL & N A mer. & & & \\
\hline HA IILTONIA & HAMILTONIA & N. Amer. & & $\mathrm{n}$ & $\mathbf{s}$ \\
\hline $\begin{array}{l}\text { oleifera Muhl. } \\
\text { Pyrularia pubera Mich. }\end{array}$ & \} American oil nut & N. Amer. & & $\mathbf{h}$ & s \\
\hline HEDERA & IVY & & & & \\
\hline $\begin{array}{l}\text { helix L. } \\
\text { HEDYCHIUM }\end{array}$ & $\begin{array}{l}\text { common } \\
\text { HEDYCHIUMI }\end{array}$ & Europe & Med. & h & s \\
\hline $\begin{array}{l}\text { coronarium Retz. } \\
\text { HEDYSARUMI }\end{array}$ & crownerl & E. Indies & & h.h & $p$ \\
\hline $\begin{array}{l}\text { HEDYSARUM } \\
\text { canalense } \mathrm{L} \text {. }\end{array}$ & $\begin{array}{l}\text { HF.DYSARUM } \\
\text { Canadian }\end{array}$ & N. A mer. & & h & $p$ \\
\hline $\begin{array}{l}\text { canescens L. } \\
\text { caput galli L. }\end{array}$ & $\begin{array}{l}\text { hoary } \\
\text { cock's head }\end{array}$ & $\begin{array}{l}\text { llo. } \\
\text { Europe }\end{array}$ & & $\begin{array}{l}\mathbf{h} \\
\mathbf{h}\end{array}$ & $\mathrm{p}$ \\
\hline coronarium L. & French Honeysuckle & & Arts & $\ddot{h}$ & a \\
\hline $\begin{array}{l}\text { gyrans L. } \\
\text { marilandicum L. }\end{array}$ & $\begin{array}{l}\text { moving plant } \\
\text { Maryland }\end{array}$ & $\begin{array}{l}\text { F.. Indies } \\
\text { N.d mer. }\end{array}$ & & $\begin{array}{l}\text { h.h } \\
\text { h }\end{array}$ & $\begin{array}{l}\text { b } \\
\text { p }\end{array}$ \\
\hline & $\begin{array}{l}\text { naked flow ering } \\
\text { Saint Foin }\end{array}$ & $\begin{array}{c}\text { do. } \\
\text { Europe }\end{array}$ & Agr. & $\begin{array}{l}\text { h } \\
\text { h }\end{array}$ & p \\
\hline pallasii IVilld. & Siberian & do. & & b & p \\
\hline paniculatum $\mathbf{L}$. & panicled & N.Amer. & & $\mathbf{h}$ & $\mathbf{P}$ \\
\hline & roc & $\mathbf{E}_{0}$ & & & P \\
\hline $\begin{array}{l}\text { viridiflorum L. } \\
\text { IIELENIUMI }\end{array}$ & $\begin{array}{l}\text { green flow ered } \\
\text { HELENIUM }\end{array}$ & N. A mer. & & & p \\
\hline $\begin{array}{l}\text { autumnale } 1 . \\
\text { HFLIANTIIUS }\end{array}$ & $\begin{array}{l}\text { sinnoth } \\
\text { SUNFLOWER }\end{array}$ & N. Amer. & & h & P \\
\hline altissimus $\mathbf{L}$. & tall & N. Amer. & & 1 & \\
\hline $\begin{array}{l}\text { annuus L. } \\
\text { decapetalus } \mathbf{L} \text {. }\end{array}$ & $\begin{array}{l}\text { common } \\
\text { ten petal'd }\end{array}$ & $\begin{array}{l}\text { S. Amer. } \\
\text { N. Ainer. }\end{array}$ & Agt. & $\begin{array}{l}\text { h } \\
\text { h }\end{array}$ & a \\
\hline $\begin{array}{l}\text { divaricatus } \mathbf{L} \text {. } \\
\text { gignuteus } \mathbf{L} \text {. }\end{array}$ & $\begin{array}{l}\text { branithing } \\
\text { giant }\end{array}$ & $\begin{array}{l}\text { do. } \\
\text { do. }\end{array}$ & & $\begin{array}{l}\mathrm{h} \\
\mathrm{h}\end{array}$ & $\mathrm{p}$ \\
\hline iuberosis J. & Jcrusalem artichoke & S. Amer. & Diet & is & ]$^{3}$ \\
\hline
\end{tabular}


HURTUS ELGINENSIS.

IHLICIIRYSUM

clurysanthum Pers. Xerantliemum hrac-

HELIO'TROP'IUM

inclicum 1.

peruviaum L.

parviftorum $\mathbf{L}$.

IIVI.LFIBOIRUS

trifolius L.

viridis $\mathbf{L}$.

IELLENIA.

allughas $\mid F i l l d$.

$\left.\begin{array}{r}\text { Heritiera allughas } \\ \text { Retz. }\end{array}\right\}$

IIEMEROCALLIS cornlea Vent.

fiava $\mathbf{L}$.

fulva 1

HEMIMEIRIS

urticifolia IVilld.

urticifolia Willd.

IERMANNIA

alnifolin $\mathbf{L}$.

althæfolia $L$.

denudata L.

liusuta Wend.

Jivssopifolia L.

salvifolia L.

scubra Cav. ?

aspura Wend. $\}$

HESPERIS

matronalis $\mathbf{L}$.

tristis 1

HEUCHERA

americann L.?

cnrtusa . ITich. $\}$

H1IIISCUS

abclınoschus L.

escuientus 1 .

graurlitorus Jich.

Iieteropulyllus Vent.

manilıot $\mathbf{i}$.

militaris C'av.?

l:evis Scop.

mosclıcutos

mutabilis $\mathbf{l}$.

palustris $\mathbf{L}$.

phoniceus I.

rosa sinensis $\mathbf{I}$.

v. A. pleno cameo

v. A. pleno coccineo

ripurits Pers.

hiastatus . Mich

virginicus $\|$ ult.

sabilarift: L.

seaber Mich.

speciosus . lit. ?

coccineus II ilt.

spiralis Cav.

sy riacus L.

\%. flore pleno

ข. folis 2 ariegatis

trionum I.

GOLDEN SUNFLOWEIR

Botany Bay

TURN SOLE

common or wild

swect

small flowered

HOLLFBORE

threeleaved-Gold thread

gireen

HELLENIA

broad leaved

DAY LILY

blue

yellow

orange

HEMIMEIRIS

\}sarlet or nettle leaved

HERMANNIA

alder leaverl

marsh mallow leaved

smooth

hairy

hyssop leaved

sage leaved

rough

ROCKET

garclen

y ellow

HEUCHERA

purple flowered

HIBISCUS

\section{musk}

Okra

large flowered.

prickly

palmated

hastated

comimon-ULres Mallow

chanceable

marsh

small flowering

China rose

double flesh coloured

double searlet

river

rarious leavel

rough

scarlet

spiral

garder or Althea frutex

double flow ered

varicgaterl leaved

bladeler Ketmin
N. Holl.

g.h b

N. A mer.

l'eru

E. Indies

Arts $\underset{\substack{\text { g.h } \\ \text { h }}}{\mathrm{h}}$ a

Med. h $p$

Europe Med. h p

B. Indies

h.h p

Tapan

Europe

China

$\begin{array}{cc}\text { g.h } & \text { p } \\ \text { h } & \text { p }\end{array}$

S. Amer. $\quad$ g.h s

C. G. II. g.h s

do. g.h s

do. g.h s

do. g.h s

do. g.h s

do. g.h 8

do. g.h 8

$\begin{gathered}\text { Europe Med. h } \\ \text { do. }\end{gathered}$
h

N. Amer. $\quad$ h $\quad p$

B. Indies Med. 1.h s

do. Diet h a

Florida g.h I'

N. Holl. g.h s

B. Indies g.h s

Louisiana $h \quad p$

N. Amer. Med. h p

Chisa g.h s

NAmer. li p

E. Indies h.li s

do. h.h s

N. Amer. h I

E. Indies h a

Creorgia g.h l'

N. Amer. h p

Mexico g.h s

Europe h a 


\begin{tabular}{|c|c|c|c|c|}
\hline virginicus $\mathbf{L}$. & Virninian & N.Amer. & & h \\
\hline $\begin{array}{l}\text { IIER ACIUM } \\
\text { aurantiacum L. }\end{array}$ & $\begin{array}{l}\text { HAWK WEED } \\
\text { orange coloured }\end{array}$ & Europe & & h \\
\hline gronarii L. & Gronovius's & N. Amer. & & \\
\hline Kalmii L. & Kalm's & do. & & h \\
\hline $\begin{array}{l}\text { marianum Willd. } \\
\text { scibrum .Mich. }\end{array}$ & $\begin{array}{l}\text { Maryland } \\
\text { rough }\end{array}$ & $\begin{array}{l}\text { do. } \\
\text { do. }\end{array}$ & & $\mathrm{h}$ \\
\hline $\begin{array}{l}\text { venosum L. } \\
\text { [OLCUS }\end{array}$ & $\begin{array}{l}\text { veined } \\
\text { SOF' GRASS }\end{array}$ & do. & & \\
\hline $\left.\begin{array}{l}\text { flagrans Willd. } \\
\text { odoratus Mich. }\end{array}\right\}$ & seneca grass & N. Amer. & Agr. & h \\
\hline $\begin{array}{l}\text { lanatus } \mathbf{L} \text {. } \\
\text { mollis } \mathbf{L} \text {. }\end{array}$ & meadow & do. & Agr. & h \\
\hline HOLOSTEUM & $\begin{array}{l}\text { creeping } \\
\text { HOLOSTEUM }\end{array}$ & Eu & & \\
\hline $\begin{array}{l}\text { cordation L. } \\
\text { OPFA }\end{array}$ & $\begin{array}{l}\text { heart leaved } \\
\text { HOPEA }\end{array}$ & W. Indies & & h \\
\hline $\left.\begin{array}{l}\text { tinctoria L. } \\
\text { Symplocos tinctoris } \\
\text { l'Her. }\end{array}\right\}$ & dyers & Carolina I & Med. & $\mathbf{g} \cdot \mathbf{h}$ \\
\hline $\begin{array}{l}\text { HORDE:UM } \\
\text { distichon L. }\end{array}$ & BARLEY & & & \\
\hline $\begin{array}{l}\text { distichon L. } \\
\text { hexastichon }\end{array}$ & $\begin{array}{l}\text { two rowed } \\
\text { six rowed-v }\end{array}$ & Tartary & $\begin{array}{l}\text { Diet } \\
\text { Diet }\end{array}$ & $\begin{array}{l}\mathbf{h} \\
\mathbf{h}\end{array}$ \\
\hline maritimun Vahl. & $\begin{array}{l}\text { sea } \\
\text { sea }\end{array}$ & Europe & $\mathrm{Agr}$. & h \\
\hline $\begin{array}{l}\text { vulgare L. } \\
\text { zeocriton L. }\end{array}$ & common & do. & Diet & h \\
\hline $\begin{array}{l}\text { zeocriton L. } \\
\text { HOUS'TONEA }\end{array}$ & $\begin{array}{l}\text { spratt ol Battledore } \\
\text { HOUSTONEA }\end{array}$ & & & \\
\hline coceinea Andr. & scarlet & Mexico & & \\
\hline $\begin{array}{l}\text { cœrulea L. } \\
\text { HUMULUS }\end{array}$ & $\begin{array}{l}\text { blue } \\
\text { HOP }\end{array}$ & ler. & & \\
\hline lupulus $\mathbf{L}$. & commion & Europe & Med. & h \\
\hline $\begin{array}{l}\text { HURA } \\
\text { crepitans L. } \\
\text { HYACINTHUS }\end{array}$ & $\begin{array}{l}\text { SAND BOX TREE } \\
\text { common } \\
\text { HYACIN'TH }\end{array}$ & S. Amer. & Arts & h.h \\
\hline botiy aides $\mathbf{L}$. & blue grape & Europe & & h \\
\hline s $\mathbf{L}$. & $\begin{array}{l}\text { puiple grape } \\
\text { fragrant }\end{array}$ & & & \\
\hline orientalis $\mathbf{L}$. & garden & & & \\
\hline $\left.\begin{array}{l}\text { racemosus L. } \\
\text { junciformis } I . m k\end{array}\right\}$ & hare bell & Europe & & h \\
\hline HYDKANGEA & HYDRANGEA & & & \\
\hline $\begin{array}{l}\text { arborescens L } \\
\text { vulgaris Mich } \\
\text { hortensis Sm. }\end{array}$ & common tree & N. Amer. & & $\mathbf{h}$ \\
\hline $\begin{array}{l}\text { hortensis Sm. } \\
\text { Hortensia rosea } J I . P \text {. } \\
\text { speciosa Pers. } \\
\text { opulnides Lmk. } \\
\text { Primula mutabilis Lour }\end{array}$ & $\begin{array}{l}\text { changeable or Japan } \\
\text { Rose }\end{array}$ & China & & g.h \\
\hline $\begin{array}{l}\text { Primula inutubilis } L \\
\text { v. flore purpureo } \\
\text { quercifolia Bartr. } 2\end{array}$ & purple flowered do. & & & \\
\hline quercitolia Bartr. $\}$ & oak leaved & Florida & & $\mathbf{h}$ \\
\hline $\left.\begin{array}{l}\text { radiata Willd } \\
\text { nivea Mich. }\end{array}\right\}$ & radiated & Carolina & & II \\
\hline $\begin{array}{l}\text { HYDROCOT YLE } \\
\text { antericana L. }\end{array}$ & $\begin{array}{l}\text { PENNY WORT } \\
\text { American }\end{array}$ & N. A mer. & & h \\
\hline $\left.\begin{array}{l}\text { repanda Pers. } \\
\text { ficarioides } \boldsymbol{M i c h} .\end{array}\right\}$ & angula' leaved & Carolina & & g.h \\
\hline $\begin{array}{l}\text { is } \mathrm{L} \text {. } \\
\mathrm{N} \text { 酐 }\end{array}$ & common & N. A mer. & & h \\
\hline $\begin{array}{l}\text { EN EA } \\
\text { rbaril L. }\end{array}$ & $\begin{array}{l}\text { LOCUST TREE } \\
\text { two leaved }\end{array}$ & W. Indies & Med. & h.h \\
\hline $\begin{array}{l}\text { HYOSCYAMUS } \\
\text { niger L. }\end{array}$ & $\begin{array}{l}\text { HENBANE } \\
\text { black }\end{array}$ & N. Amer. & Med. & h \\
\hline IYPEIICUM & ST. JOIIN'S WORT & & & \\
\hline $\begin{array}{l}\text { canarleuse I. } \\
\text { elatum . Iit. }\end{array}$ & $\begin{array}{l}\text { Canadiau } \\
\text { eall }\end{array}$ & $\begin{array}{l}\text { A mer. } \\
\text { do. }\end{array}$ & & 1 \\
\hline
\end{tabular}


HORTUS LLGINENSIS.

liiısutum L.

maeulatum Mich.

hairy

spotted

monogyum I.

Chinese

nudiflorum Mich.

naked flowered

parviflorum Willd. $\}$ five nerved

perforatum L. commou

prolificum $\mathbf{L}$.

quarlrangulum L.

prolific

setosum L.

virginicum $\mathbf{L}$.

HYTOTHARIS

rarlionta L.

HYI'OXIS

erecta $I$.

HIYSSOPUS

officiualis L.

JASMINUM

azoricum L.

fiuticans $\mathbf{L}$.

grandiflorum L.

square stalked

bristly

Virginian

CA'I'S EAll

long lonted

IIYPOXIS

upright

HYSSOP

cominon

JASMINE

Azorian

yellow

Catalonian

humile $\mathbf{L}$.

odol'atissinum L.

oflicinale $\mathbf{L}$

dwarf vellow

sweet seented

white

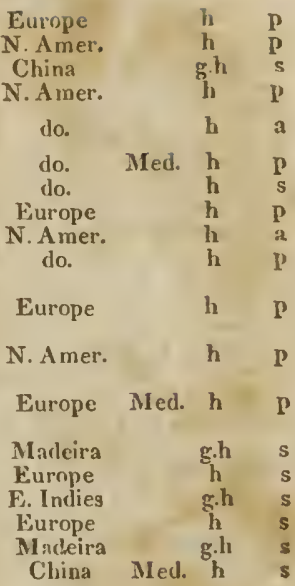

Sambac Ait.

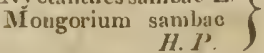

Arabian

E. Indies

g.h s

simplicifolium Forst.

austiale Pers.

JAT'ROP'HA

cureas L.

multifida $\mathrm{L}$.

IBËRIS

saxatilis L.

umbellata $\mathbf{L}$.

ILE: $X$

aquitolium $\mathbf{L}$.

canarlensis L.

opaca Ait.

vomitoria Ait.

Cassine peragua L. $\}$

IL.LECERRUM

capitatum $\mathbf{L}$.

echinatum Desf.

ficoirleum $\mathbf{L}$.

ILLICIUMI

parviflorum .Hich.

IMPATIENS

balsamina $\mathbf{L}$.

biflora Wult.

INDIG;OFF.RA

simple leaved

PHYSIC NUT

angular leaved

cut leaved

CANDY TUE'T

rock

common

IIOLLV

common

Canarlian

Carolinian

South Sea tea

N. Holl.

g.h s

S. Amer. Med. h.h s do. Med. l.h s

$\begin{array}{lll}\text { S. Europe } & \text { g.h } & \text { s } \\ \text { Europe } & \text { h } & \text { a }\end{array}$

Europe Med. h $t$

N. Aner. $\quad h$ t

Florida Med. g.h $t$

KNOT GRASS

headed

prickly

trailing

ANISEED TREE

y cllow flowered

BILSAM

conimon

in o flowered

INDIGO

argentea l'Her.

glallea $1 . m k$.

frutescens $\mathbf{L}$.

sericea $\mathbf{L}$.

timetoria $\mathbf{L}$.

silvery

shrubby

gilky

cominon

ING.I

$\left.\begin{array}{l}\text { ING. Milla. } \\ \text { MIMOSA Anct. }\end{array}\right\}$

micropliy lia Jumb.

purpurea Willd.

unguis enti Willd.

INUiA

crithmifolia $\mathbf{L}$. small leared

purple

four leaved or cut's paw

ELECAMHANE

samphire leared $\begin{array}{lll}\text { Europe } & \text { h } & \text { p } \\ \text { Barbary } & \text { h } & \text { a }\end{array}$

S.Amer. g.h P

Florida g.h s

$\begin{array}{llr}\text { E. Indies } & \text { h } & \text { a } \\ \text { N. Amer. } & \text { hl } & \text { a }\end{array}$

E. Indies h.h s

C. C. H. g.h s

do. g.h s

E. Indies Arts h.lı s

$\begin{array}{lll}\begin{array}{l}\text { S. Amer. } \\ \text { W. Iudies } \\ \text { do. }\end{array} & \text { h.h } & \text { s } \\ \text { h.h } & \text { s } \\ \text { F.h } & \text { h.h } & \text { s } \\ & \text { hr } & \text { p }\end{array}$ 


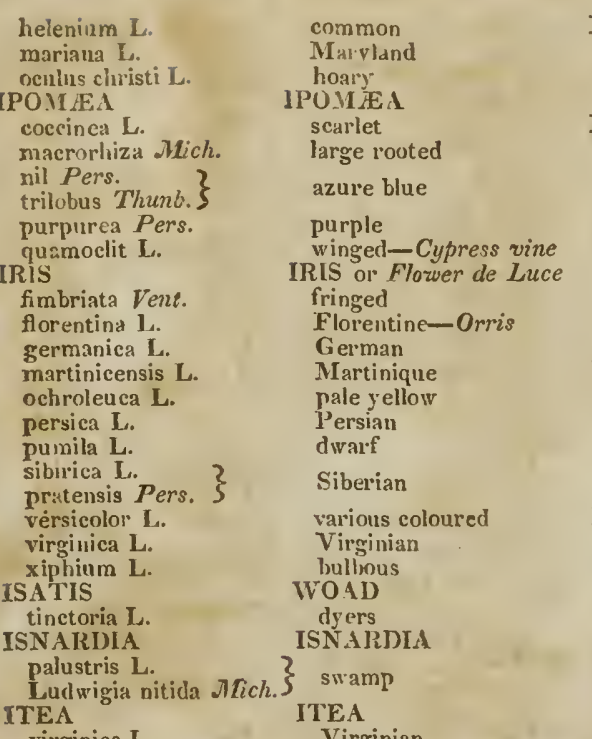

IVA virginica $\mathbf{L}$

fivutescens $\mathbf{L}$.

3 UGLANS

alba $\mathbf{L}$.

tomentosa Jich.\}

cinerea L.

cathartica Mich $f\}$

compressa Gert.

alba Mich.

squamosa Mich f

glabra Mhul.

porcina Mich.f.

nigra $\mathrm{L}$.

obcordata Muhl. $\}$ amara Jlich.f. olivæformis Mich. angustifolia Sit. pecan Walt. cylindrica $L m k$ rubra Gert.

regia $\mathbf{L}$.

sulcata Willd. mucronata . Hich. $\}$

JUNC.US

bufonius $L$.

effisis $\mathrm{L}$.

nodosus I.

pilosus L.

squartosus $\mathrm{I}$.

sylvaticus L.

tenuis Hilld.

JUNIPEIRUS

bermudiana I.
Virginian

IV A or Bastard Jesuit's shrubby

WALNUTT or HICKORY

white heart

butter nut-white

shell bark

pig or hog nut

black

bitter nut

pecan or Illinvis nut

N. Amer.

Med. $\stackrel{g}{h} \quad$ p

Med. g.h P

Europe do.

W. Indies

Europe

Persia

Europe

Med. I

Med. II

Europe

P

do.

N. Amer Med h Amer. Med. h

Europe

h $p$

h

h

h

virope Arts h o

N. Amer.

Bark

N. Amer. h s

N. Amer. Arts h t

do. Med.\& h

Arts

do. Diet\& li $t$

Arts

do. Arts $h$ t

do. Arts \& h

Med.

do. Arts h

do. Diet h t

common-Madeiranut Persia Diet $h_{\mathrm{h}} \mathrm{t}$

furrowed

RUSII GRASS

toad

soft

knotty

hairy

moss or Goose corn

wood

slendlev.

IUNII'ER or Cedar

Bermurian
N. Amer. Arts li t

N. Amer.

do. $h$ p

do. h p

clo. h p

do. h

do. h p

do. $\mathrm{h}$ p

VI. Indies Arts $h \quad t$ 
communis $\mathbf{I}$.

эxycedrus $\mathbf{L}$.

plienicea $\mathbf{L}$.

piostrat: Mich. ?

repens Donn. $\}$

salsina L.

virginiana $\mathbf{L}$.

JUSTIC:IA

aclliatuda L.

betonica $\mathbf{L}$.

eoccinea Ait.

comata J Tahl.

piet:ı I.

peruviana Car.

scorpioiles L.

sexangularis $\mathbf{L}$.

IXIA

bulbocodium $\mathbf{L}$.

maculata Thunb. polystaehya $\mathbf{L}$.

IXORA

coccinea $\mathbf{L}$.

K IEMPEKIA

galanga $\mathbf{L}$

rotuncla $L$.

KA L.MIA

angustifolia $\mathbf{L}$.

glauea Ait.

hirsuta Bartr.

Iatifolia L.

kirIGIA

virginica Willd.
Hyoseris virginica L. $\}$ Virginian

LACTUCA

erispa Roth.

elongata Muhl.

sativa L.

L.AGASCA

mollis Cav.

LAGERSTREMM indien $\mathrm{L}$.

LA MIUM

amplexicaule $\mathbf{L}$.

garganicum $\mathbf{L}$.

LANA NA

aculeata $\mathbf{L}$.

camara L.

involuerata $\mathbf{L}$.

trifolia L.

LATHYRUS

clymenum $\mathbf{L}$.

latifolius $\mathbf{L}$.

monanthos L.

Vicia monanthus $P$ ers.

nissolia L.

oloratus L.

sativus L.

sphxricus Roth. ?

axillatis $L m k$.

tingitanus $\mathbf{L}$. eommon

brown berried

Plicnicean

ereeping

Savin

red!

JUSTICIA

Malabar nut

blue flowered

searlet.

whorled

painted

Peruvian

revolute

six ungled

IXIA

European

spotted

miny spiked

IXORA

scarlet

GALANFALE

ofticinal

l'ound rooted

KALMIA or Laurel

narrow leaved

glau cous

hairy

broad leaved

KRIGIA

Virginian

curled

narrow leaved

common

LAGASCA

soft

LAGERSTRENIA

Indian

DEAD NETTLE or IIenbit

common

woolly

LAN'TANA

prickly

various coloured

round leaved

three leaved

LATHYRUS

various flowered

everlasting pea

one flowered

crimson

sweet pea

ehickling verch

spherical

Tangier pea
Europe

Arts \& I

Merl.

do.

do.

N. Amer.

Europe Merl. h

N. Amel. Arts h

E. Indies

S. Ámer.

g.h

W. Iudlies

F. Indies

Peru

W. Indies do.

h.h

$s$

h.h p

h.h s

h.h

h.h

h.h

S. Europe

C. G. H.

do.

g.h p

g.h p

E. Indies

h.h 6

E. Indies Med, h.h do. Med. h.h

N. Amer, do.

Carolina

N. Amer. Med. $h$ s

N. Amer.

h a

$\begin{array}{lll}\text { N. Amer. Diet } & \mathbf{h} & \mathbf{a} \\ \begin{array}{c}\text { Diet \& } \\ \text { Med. }\end{array} & \mathbf{h} & \mathbf{b} \\ & \mathbf{a}\end{array}$

S. Amer. li.h a

E. Indies h.h a

N. Amer. h a

Europe h p

W. Indies h.h s

do. h.h s

do. h.h s

do. h.h s

Levant h a

Europe h p

Russia h a

Europe h a

Sicily Agr. h a

do. h a

Barbary h a 


\section{LAVA NDULA} spica I.

LAVATERA

arborea $\mathbf{L}$

maritima Caz.

olbia L.

thuringiaca L.

trimestris L.

unguiculata $H . P$.

tomentosa Dumont $\}$

\section{LAURUS}

xstivalis $\mathbf{L}$.

camphora L.

caroliniensis Mich.

cinnamomum $\mathbf{L}$

geniculata Mich. $\}$

axillaris $\mathbf{L m k}$.

nobilis L.

persea L.

psendo benzoin Mich.?

benzoin L.

sassafiras $L$.

LAIVSONIA

iner'mis $\mathbf{L}$.

LEBECKIA

cytisoidles Thunb.

$\left.\begin{array}{l}\text { cytisnidles Thunb. } \\ \text { Spartium cytisoides Ait. } \\ \text { Fuenus capensis L. }\end{array}\right\}$ Cytisus Icaverl

LECHEA

major $\mathbf{I}$.

minor L.

LEON'TICE

thalictroiles $\mathrm{L}$. Caulophyllum thalic-

\section{LEERSIA}

oryzoides Irilld.

l'halaris oryzoides L. $\}$ rough

LEUNTODON

taraxacim L.

DA NIELION

common

\section{LFONURUS}

en riaca L.

crispus L.

marrubiastinin $\mathbf{L}$.

L.EPECHINIA

$\left.\begin{array}{l}\text { spieata Willl. } \\ \text { Horminum caulescens }\end{array}\right\}$

LEPIDIUM

latifolium $\mathbf{L}$.

satirum $\mathbf{L}$.

suffruticosum $\mathbf{L}$.

virginicum $\mathbf{L}$.

LEPTOSPF]RMUM

scoparium Sin.

Mela'euca senparia

Mend.
Jenderia

MOTHER IVORT

common

curled

small fowerer

LEPECHINIA

Philadelplus scoparius Dittander
grarden cress

shrubby Virginian
Europe Med. Is

Europe b b

S. Europe g.h s

do. g.h s

Europe h h

g.h s

$\underset{\text { Japan }}{\text { N.Amer. Med. } \mathrm{g} \cdot \mathrm{h}} \mathrm{t}$

Carolina g.l $t$

E. Indies Med. $\mathrm{h} . \mathrm{h}$ t

N. Amer. ht t

S.Europe Med. g.h t

S. Amcr. Diet g.l t

N. Amer. Med. h t

do. Med. h t

E. Indies Arts\& h.h s

Med.

C. G. H

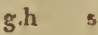

N. Amer.

do.

h p

N. Amer. Med. h p

N. Amer.

h

N. Amer. Diet \& h p Med.

V. Amer. Med. /

Siberia h

Europe

h

Mexico h a

Europe Med, h p do. Med.\& h a

S. Furope

N. $\Lambda$ mer.

g.h s SUUTH SEA MYRTLE

న. Holl.

g.h s 
HORTUS ELGINENSIS.

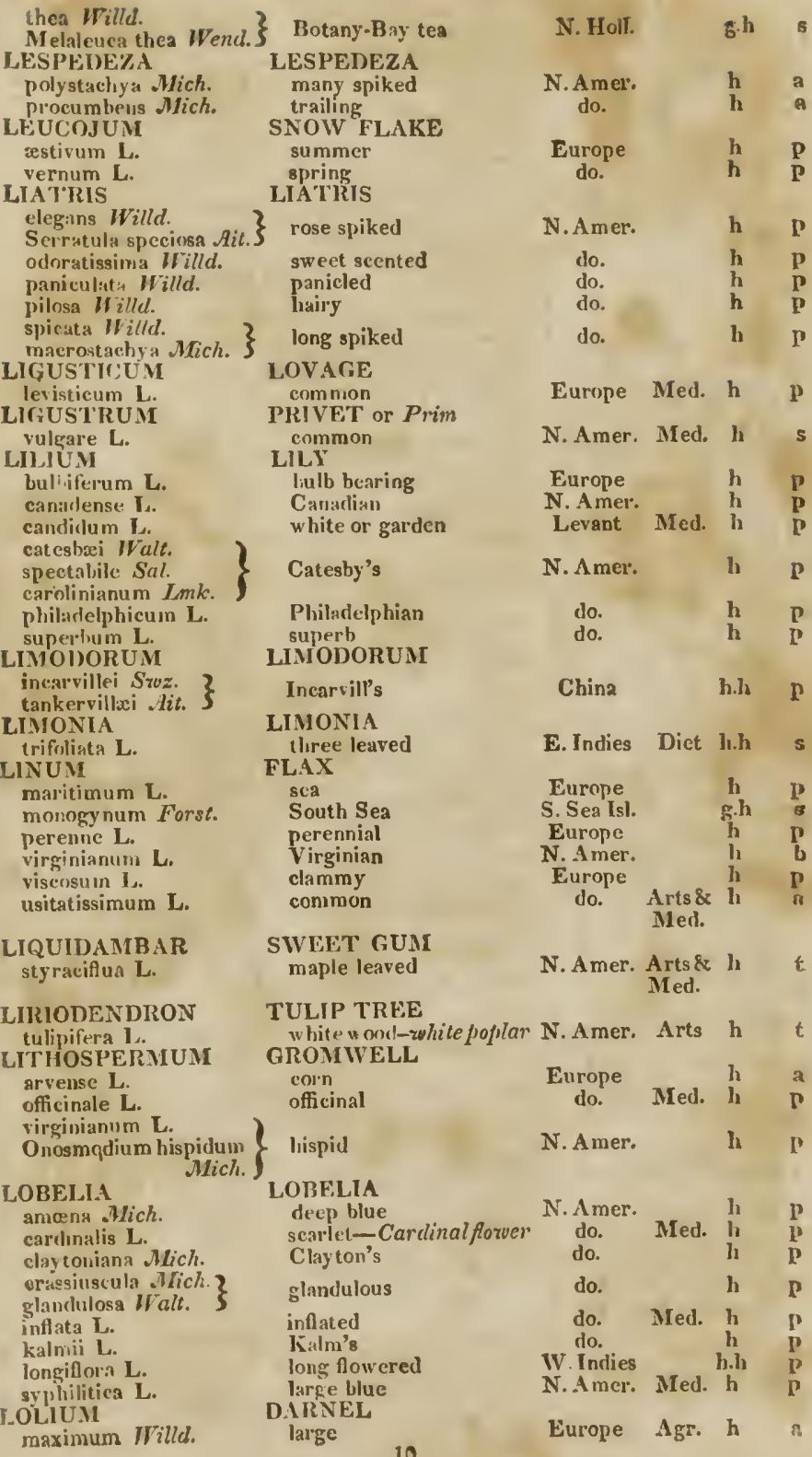


HORTUS ELGINENSIS.

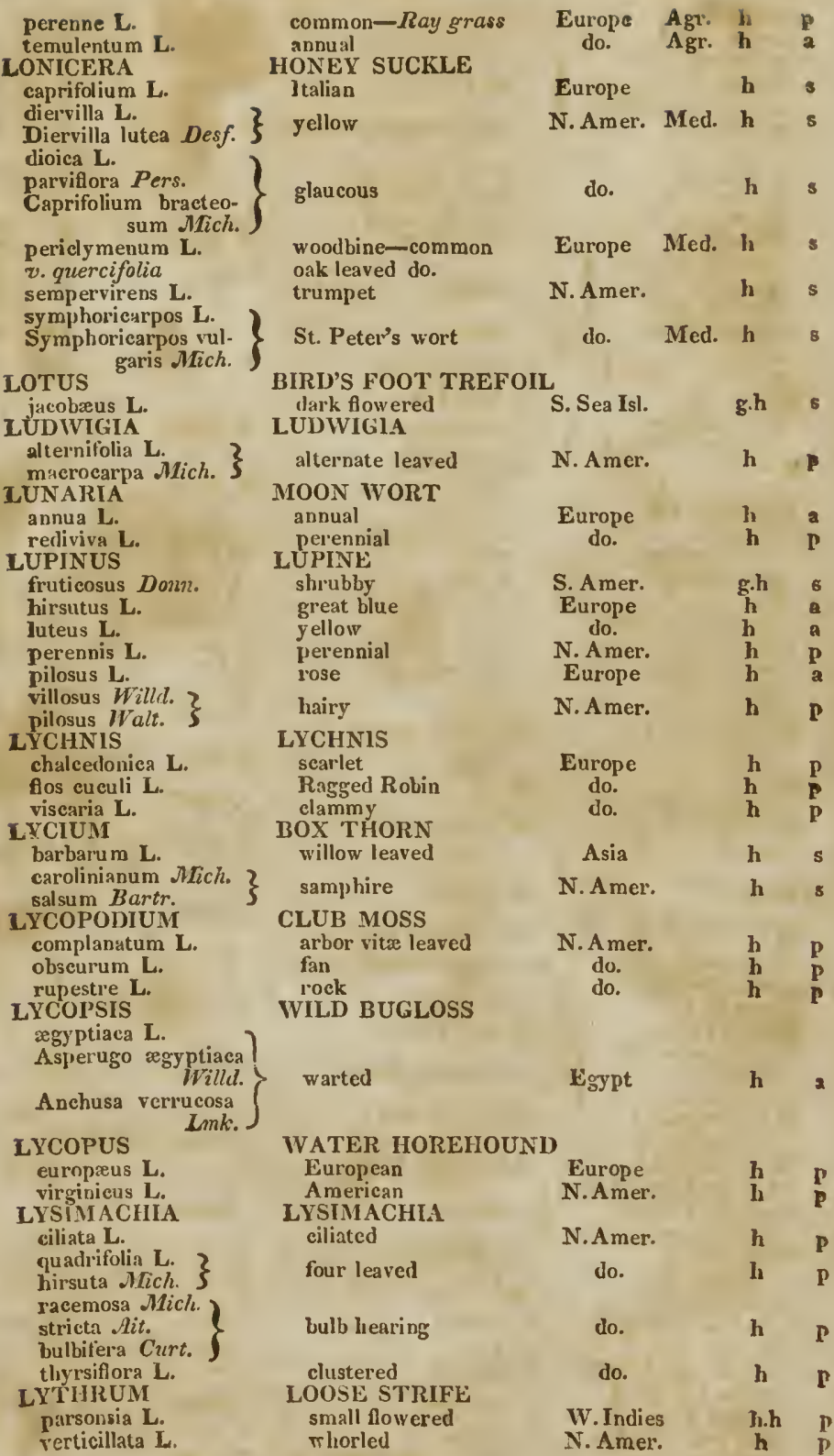


MIANOLIA

acuminata $\mathbf{L}$.

auriculata $\mathbf{I m k}$.

fraseri Walt.

fraseri Walt.
pyramidata Bartr.
glauca L.

ข. latifolia

v. longifolia

grandiflora L.

macrophylla Mich.

obovata Thumb.

purpurea Curt.

discolor Vent.

pumila Andr.

tripetala L.

umbrella $\mathbf{L} m k$. $\}$

MALAXIS

liliifolia Szoz.

Ophrys liliifolia L.

MALVA

americana $\mathbf{L}$.

caroliniana $\mathbf{L}$.

crispa L.

fragrans Jacq.

moschata L.

parvifora $\mathbf{L}$.

polystachya Cav.

rotundifolia $\mathbf{L}$.

scoparia Cav.

spicata L.

virgata Cav.

MANGIFERA

indica $\mathbf{L}$.

MANULEA

oppositifolia Tent.

MARA NTA

arundinacea $\mathbf{L}$.

EIAICA

palurlosa Willd.

MARIRUBIUM

alyssum $\mathbf{L}$.

candidissimum L.

vulgare L.

MARTYNIA

proboscidea Ait. arnua $\mathbf{L}$. alternifolia $\operatorname{Lmk}$.

MATRICARIA chamomilla $\mathrm{L}$.

MAURANDIA

semperflorens Jacq. scandeus Pers.

Ustera scandens Cav.

MEDEOLA

virginica $\mathbf{L}$.

MEDICAGO

aculeata Willd.

arborea $\mathrm{L}$.

denticulata Willd.

clegans Jacq. 2

rugosa $l m k$. $\}$

glutinosa Willd.

Iupulina

\section{MAGNOLIA}

Cucumber tree

car leaved

swamp sassafras-Beaver N. Amer. tree

broad leaved do.

long leaved do.

large flowered

large leaved

purple

dwarf

umbrella tree

MALAXIS

lily leaved

MALLOW

American

creeping

curled leaved

swect

musk

small fowered

many spiked

round leaved

yellow upright

spiked

slender

MANGO TREE

Indian

MANULEA

opposite leaved

ARROW ROOT

Indian

MARICA

marsh

HOREHOUND

plaited leaved

white

common

MARTYNIA

Cuckold's horns

N.Amer.
Carolina
N. Arner.

Carolina

N.Amer.

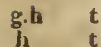

Chinz

g.h s

do.

g.h 6

N. Amer.

h $t$

N. Amer. h p

N. Amer. $\quad$ h a

do. h a

Syria h a

C. G. H.

Europe h P

Africa h a

Peru g.h s

N. Amer. Diet $h$ a

Peru g.h s

W. Indies g.h s

C. G. H. $\mathrm{g} \cdot \mathrm{h}$ s

E.Indies Diet h.h t

C. G. H. g.h s

S. Amer. Med.\& li.h P

Diet

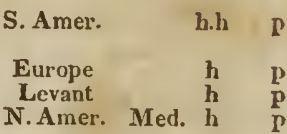

N. Amer. h

MATRICARIA

wild chamomile

MAURANDIA

climbing

MEDEOLA

Cucumber root

MEDICK

prickly

tree

toothed

crenated

clammy

black-vone-sucht
Europe Med. h a

N. Amer. Diet h a

$\begin{array}{lcc} & h & \text { a } \\ \text { Italy } & \text { g.h } & \text { s } \\ \text { Europe } & \text { h } & \text { a }\end{array}$

Sicily h a

Asia $\quad h \quad p$
Mexico g.h p 


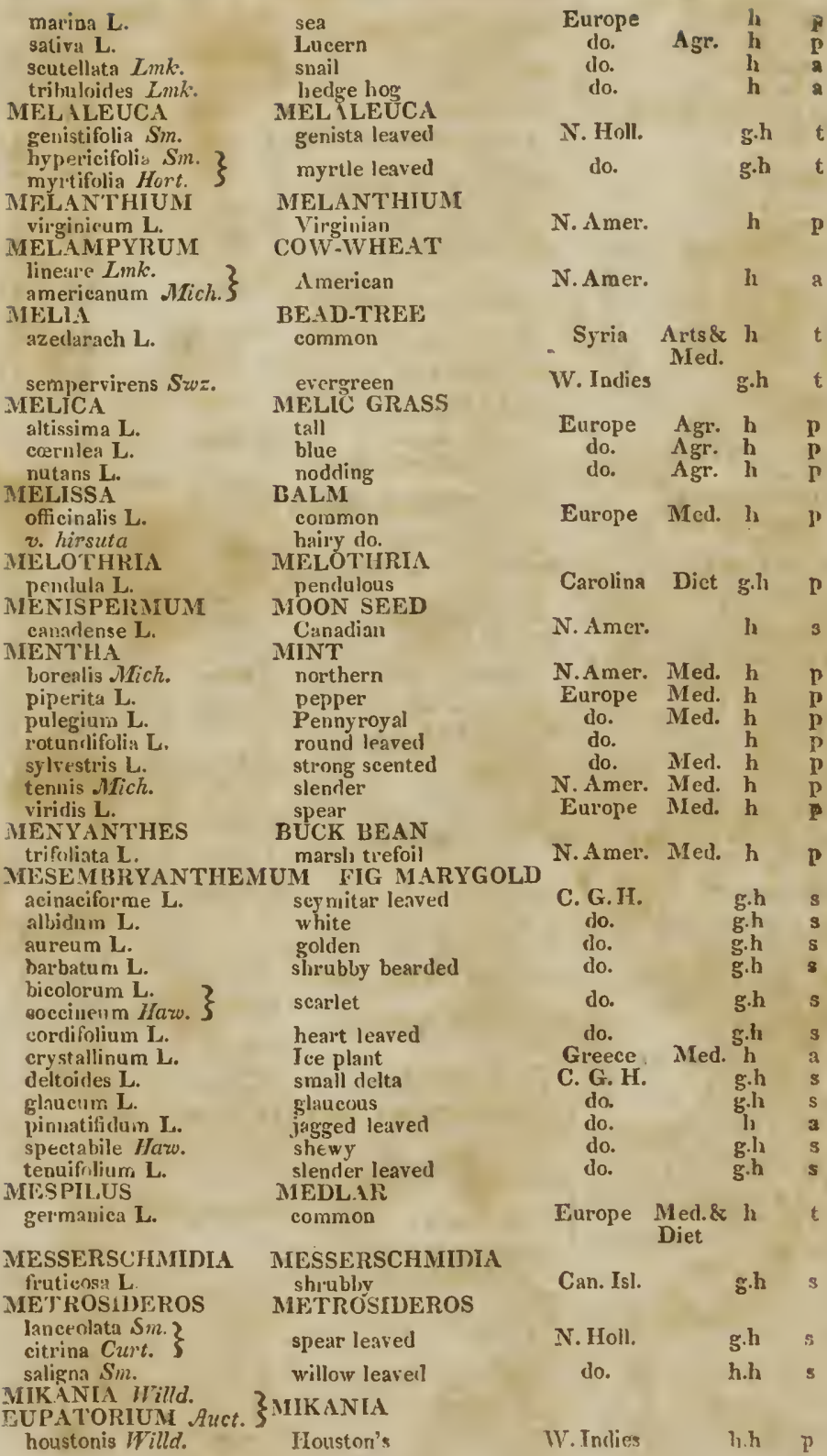


HORTUS ELGINENSIS.

seandens Willd.

MILIUM

effiısum L.

MIMOSA

concinna Filld

saponaria Rox. $\}$

purlica L.

sensitiva $\mathbf{L}$

MIMULUS

alatus Ait.

ringers $\mathbf{L .}$

MIMUSOPS

el'ngi l.

MIRA BIL.IS

jalapa $\mathbf{L}$.

Inıgiflora L.

MITCHELLA

repens $L$.

MOLLUGiO

vertieillata L.

MOLUCELLA

lævis $\mathrm{I}$.

MO.NORDICA

balsanina L.

luff: L.

MONA RDA

dislynu $I$.

fistulosa L.

MORAEA

chinensis L. chinensis L.
Ixia chincusis Ait. $\}$
gladiata L.

MORUS

alba $\mathrm{L}$.

nigra $\mathbf{L}$.

rubra L.

MUHLENBERGIA

diffusa $S c h r$.

Dilepy rum minutiflo.

MUSA rum .Mich.

sipicntum L.

MYAGRUMI

perfoliatum $\mathbf{L}$.

MYUSOTIS

lappula L.

scorpioides $L$.

virginiana L.

MYIRICA

caroliniensis IVilld.

ccrifera L.

MYRTUS

communis $\mathbf{L}$.

v. boetica

v. belgica

v. micronata

v. romana

tomentosa Ait.

NA PA: 1

levis $\mathbf{L}$.

sci.hम L.

NARCISSUS

bicolor $L$. climhing

MILLET GRASS

common

MIMOSA

lrandsome

humble plant

sensitive plant

MONKEY I'LOWFR

wiuged stcmmed

gaping flowered

MIIUSOPS

osal leaved

MARVEL OF PERU

cominion-four o'Clock

long flowered

MITCHFLLA

creeping

MOLLUGO

whorled leaved

MOLUCC;A BALM

smootl

MOMOR

conimon

Egyptian

N. Amer. Med. h p

Europe Agr. h p

E. Indics h.h

S. Amer. h.h s

do. l.ls s

$\begin{array}{lll}\text { N.Amer. } & \text { h } & \text { p } \\ \text { do. } & \text { h } & \text { p }\end{array}$

E. Indies h.h

Mexico h a

do. h a

N. Amer. Med. h s

N.Amer. li a

Syria Med. h a

do. Mck. h a

NA or Mountain Mint

Oswego tea

Rohin run-away

N. Amer. Med. h

MORAA

Chinese

sword leaved

MULBERRY

white

common

do.

Med.

China

C. G. H.

h p

China Diet lo $t$

Europe Diet\& is

Med.

American

N. Amer. Diet h $\mathbf{t}$

MUHLFNBERGIA

small flowcred

MUSA

Banana trce

GOI.D OF PLEASURE perfoliate

SCORI'ION GRASS

mai'sh

prickly seeded

Virginian

N. Amer.

h p

CANDLE BERR

large seeded

wax bearing

MYRTLE

colnmon

broad leared

narrow leaved

small leaved

roman

woollv

NAPEA

smooth

rough

N. IRC,ISSUS

two coloured
W. Indies Diet h.h p

Europe h a

N. Amer. h a

lo. h a

do. $\mathrm{E}$ or Bay berry

N. Amer. Arts h s do. Arts $h$ s

S. Europe Med. g.h s

China g.ls 3

N. Amer. h p

clo. h p

Europe h ? 
HORTUS ELGINENSIS.

biflorus Curt.

two flowered

jonquilla $\mathbf{L}$.

Jonquil

odorus L.

poeticus L.

pseurlo narcissus $\mathbf{L}$.

sweet smelling

poet's

tazett'a L.

Diffodil

Polvanthus

NEOTTIA

NEOTTIA

Ophrivalis - stivalis Mich $\}$ summer

cernua Willd.

Ophrys cernita L. $\}$

drooping-Ladies' traces

pubescens Willd

Satyrium repens.Kich.\} variegated

NEPETA

cataria L.

melissæfolia $H . P$.

NERIUM

coronarium Jacq.

v. fore pleno

odorum Ait.

v. A. pleno

oleander L.

v. Rore pleno

v. fore roseo

NEPIIRODIUM Mich.?

\section{POLYPODIUM L}

acrostichoides Mich. asplenioides Mich.

cristatum . Mich.

filix femina Nich.

marginale Mich.

noveboracense

punctilnbulum Nich.

rufidulum Aich.

tenue Mich.

thelypteroides Mich.

NICOTIANA

crispa $\boldsymbol{H} . \boldsymbol{P}$.

fruticosa $L$.

glutinosa L.

paniculata L.

rustica $\mathbf{L}$.

tobaccum L.

tomentosa Pers.

undulata Vent. $?$ odorata Donn. $\}$

NIGELLA

damascena $L$.

sativa L.

NYMPHAA

advena Ait.

lutea $\mathbf{L}$.

odorata Ait.

NYSSA

biflora .Mich.

aquatica $\mathbf{L}$.

integrifolia Ait.

CATMINT or Catnep

cominon

balm leaved

OLEANDER or Rose-bay

broad leaved

double flowered do.

sweet scented

double flowered do.

common

double flowered

rose coloured

\section{SSHIELD FEIRN}

terminal

spleen w ort leaved

crested

female

marginal

New-York

small flowered

brown flowered

slender

marsh

TOBACCO

curled leaved

shrubby

clammy

panicled

common

Virginian

Harannalx

waved leaved

FENNEL FLOWER

common

small flowered

WATER LILY

striped lowered

sellow

sweet scented

TUPELO TREE

water-Sour gum

villosa Nich.
rough-Iepperidge

$\begin{array}{ccc}\text { Eurepe } & \text { h } & \mathbf{p} \\ \text { do. } & \text { h } & \mathbf{p} \\ \text { do. } & \mathbf{h} & \mathbf{p} \\ \text { do. } & \mathbf{h} & \mathbf{p} \\ \text { do. } & \mathbf{h} & \mathbf{p} \\ \text { do. } & \mathbf{h} & \mathbf{p}\end{array}$

N. Amer. h P

do. $h \quad p$

do. Med. h p

N. Amer. Med. h p

Europe $h$ p

E. Indies g.h s

do.

g.h s

S. Europe g.h t

N. Amer. h p

do.

do.

do.

do.

do.

do.

do.

do.

do.

$\begin{array}{ll}\mathbf{h} & \mathbf{p} \\ \mathbf{h} & \mathbf{p} \\ \mathbf{h} & \mathbf{p} \\ \mathbf{h} & \mathbf{p} \\ \mathbf{h} & \mathbf{p} \\ \mathbf{h} & \mathbf{p} \\ \mathbf{h} & \mathbf{p} \\ \mathbf{h} & \mathbf{p} \\ \mathbf{h} & \mathbf{p} \\ \mathbf{h} & \mathbf{p}\end{array}$

S. Amer.

h a

China g.h s

S. Amer. $h$ a

do. $h$ a

N. Amer. Med.\& h a

do. Mrts $\mathrm{h}$,

Arts

S. Amer. Med.\& h.h s

Arts

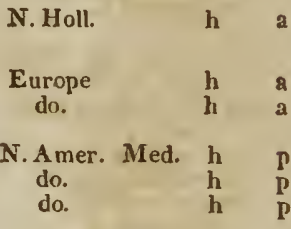

N. Amer. Arts h $t$

do. Arts $h$ t 


\section{DCHROMA}

lagopus Swz. Hombax pyramidak $\}$

\section{OCYMUM}

basilicum L.

minimum L.

monachorum L

tenuiforum L. this riflorum L.

\section{OENO'THERA}

biennis $\mathrm{L}$.

fruticosa L.

grandiflora Ait.

muricuta L.

odorata Jacq.

parvifor: 1. .

$\left.\begin{array}{l}\text { purpuluca Curt. } \\ \text { humilis Donn }\end{array}\right\}$

rosea Ait. ?

rubra Cav. $\}$

sinuata Mur.

tetraptera Cav.

\section{OLEA}

americana $\mathbf{L}$.

europæa L.

v. buxifolia fragrans Thunb.

ONOCLEA sensibilis $\mathbf{L}$.

ONONIS

cherleri L.

fiuticosa $\mathbf{L}$.

ONOPORDUM

acaulon $L$.

arabicum I.

illvricum $\mathbf{L}$.

ORCHIS

blephariglottis Willd.

ciliaris $\mathbf{L}$. spectabilis L.

ORIGANUM

agyptiacum L.

dictamnus $\mathbf{L}$.

heracleoticum L.

marjorana $\mathbf{L}$.

marjoranoides IIilld.

vulgare $\mathbf{L}$.

ORNITHOGALUM

aureum Curt.

pyramidale L.

umbellatum L.

ORNITHOPUS scorpioites $\mathbf{L}$.

OROB A NCHE uniflora $\mathbf{L}$. virginiana I.

ORONTIUM

aquaticum $\mathbf{L}$.

ORTEGIA

hisprnia $\mathrm{J}_{\text {. }}$

\section{OCHIROMA}

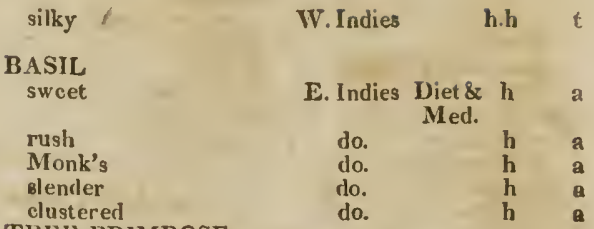

TREE PRIMROSE

common

perennial

large flowercd

prickly

sw ett scented

small flowered

purple

rose flowered

scollop leaved

square capsuled

OLIVE

American

European

box leaved do.

sweet scented

ONOCLEA

sensitive fern

REST HARROW

dwarf

shrubby

CO'TTON THISTLE

dwarf

Arabian

Illyrian

ORCHIS

white

orange coloured

showy

MARJORAM

Egyptian

Dittany of Crete

winter

sweet

N. A mer. Med.

do.

do.

do.

S. Amer.

N. Amer.

do.

h b

do.

Peru

a

a

g.h P

S. Amer. $h$ a

$\begin{array}{lll}\text { Carolina } & \text { g.h } & t \\ \text { S. Europe Med.\& } & \text { g.h } & \mathbf{t}\end{array}$ Diet

China Arts gh $t$

N. Amer. h p

$\begin{array}{cc}\begin{array}{c}\text { Europe } \\ \text { do. }\end{array} & \text { h }\end{array}$

$\begin{array}{ccc}\text { Europe } & \mathbf{h} & \mathbf{b} \\ \text { do. } & \mathbf{h} & \mathbf{b} \\ \text { do. } & \mathbf{h} & \mathbf{b}\end{array}$

N. Amer. $\quad$ h $\quad p$ $\begin{array}{lll}\text { do. } & \mathbf{h} & \mathbf{p} \\ \text { do. } & \mathbf{h} & \mathbf{p}\end{array}$

\begin{tabular}{|c|c|}
\hline $\begin{array}{l}\text { Egspt } \\
\text { Crete }\end{array}$ & Med $\stackrel{\text { g.h }}{g}$ \\
\hline $\begin{array}{l}\text { Crete } \\
\text { Greece }\end{array}$ & Merl. $g . h$ \\
\hline Portugal & $\begin{array}{l}\text { Med.\& h } \\
\text { Diet. }\end{array}$ \\
\hline 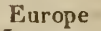 & Med. \\
\hline
\end{tabular}

C. G H. g.h $p$

Europe $\quad \stackrel{g}{h} \quad p$ do. h p

umbel flowered

BIRD'S FOOT

purslane leaved

BROOV RAPE

Europe

h p

one flowered

Virginian-Cancer Root

ORONTIUM

water

ORTFGIA

Spanish

N.Amer. $h$ p do. Med. h p

N.Amer. Med. h $\mathbf{p}$

Spain li $\mathrm{p}$ 


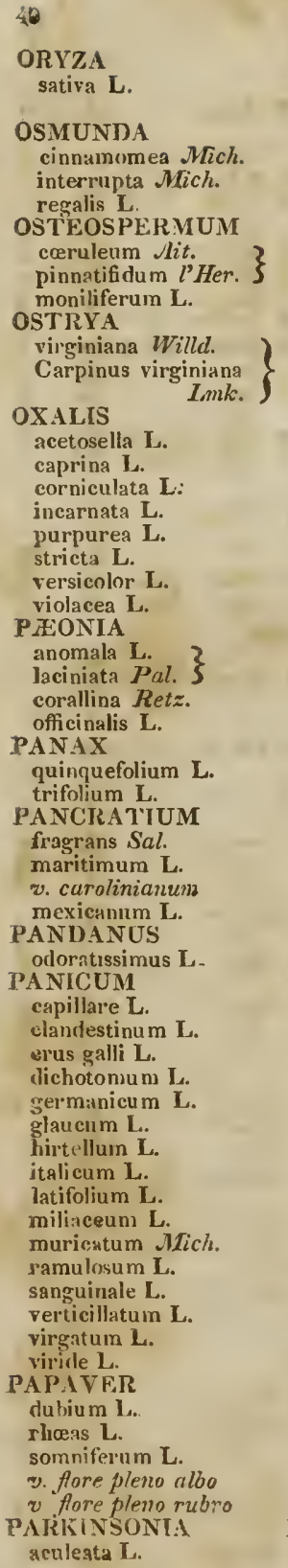

HORTUS ALGINGNSI3.

RICE

cultivated

E. Indies Arts\& h a

OSMUNDA

woolly

interrupted

flowering fern

BONE SEED

blue flowered

poplar leaved

HOP HOLNBEAM

Virginian

Med.

WOOD SORREL

common

goat's foot

procumbent

flesh coloured

purple

upright

striped flowered

violet coloured

PEONY

jagged leaved

N. Amer.

do.

do.

$\begin{array}{ll}\text { h } & \text { p } \\ \text { h } & \text { p } \\ \text { li } & \text { p }\end{array}$

C. G. H.

g.h

do.

g.h s

N. Amer. Arts h t

entire leaved

common

GINSENG

officinal

three leaved

PANCRATIUM

firagrant

Sea Daffodil

Carolinian do.

Mexican

SCREW PINE

sweet scented

PANIC GIRAS

hair

hidden flowered

cock's foot

divided branched

German

glancous

rough

Italian

broad leaved

inillet

rough seeded

branching

bloorly

whorl flowered

river

green

PÖPPY

small

corn

oflicinal or garden

double white flowered

double red do.

I'ARKINSONLA

nricklr

N. Amer. Med. h p

C. F. H.

$\begin{array}{ll}\text { g.h } & \mathrm{p} \\ \mathrm{h} & \mathrm{p}\end{array}$

$\begin{array}{lll}\text { N. Amer. } & \text { h } & \text { p } \\ \text { C. G. H. } & \text { g.h } & \text { p }\end{array}$

do.

g.h p

N. Amer. MLed. h p

C. G. H. g.h p

N.Amer. h p

Siberia h p

do. Med. $h$ p

S. Europe Med. h p

N. Amer. Med. h p do.

h $\mathrm{P}$

S. Amer. h.h $p$

S. Europe g.h I

Carolina g.h p

Mexico g.h p

B. Indies h.h s

N. Amer. Agr. h a

do. Agr. $h$ p

do. Agr. h a

do. Agr. h a

Europe Agr. h a

N. Amer. Agr. h a

W. Indics Agr. h a

Europe Diet h a

N. Amer. Agr. h p

F. Indies Med. h

N. Amer. Agr. h a do. Agr. h

do. Agr. h a

Furope Agr. h a

N. Amer. Agr. h p do. Agr. h a

Europe h a

do. Med. $h$ a

Levant Med. h a

IV. Indies h.h 
HORTUS ELGINENSIS

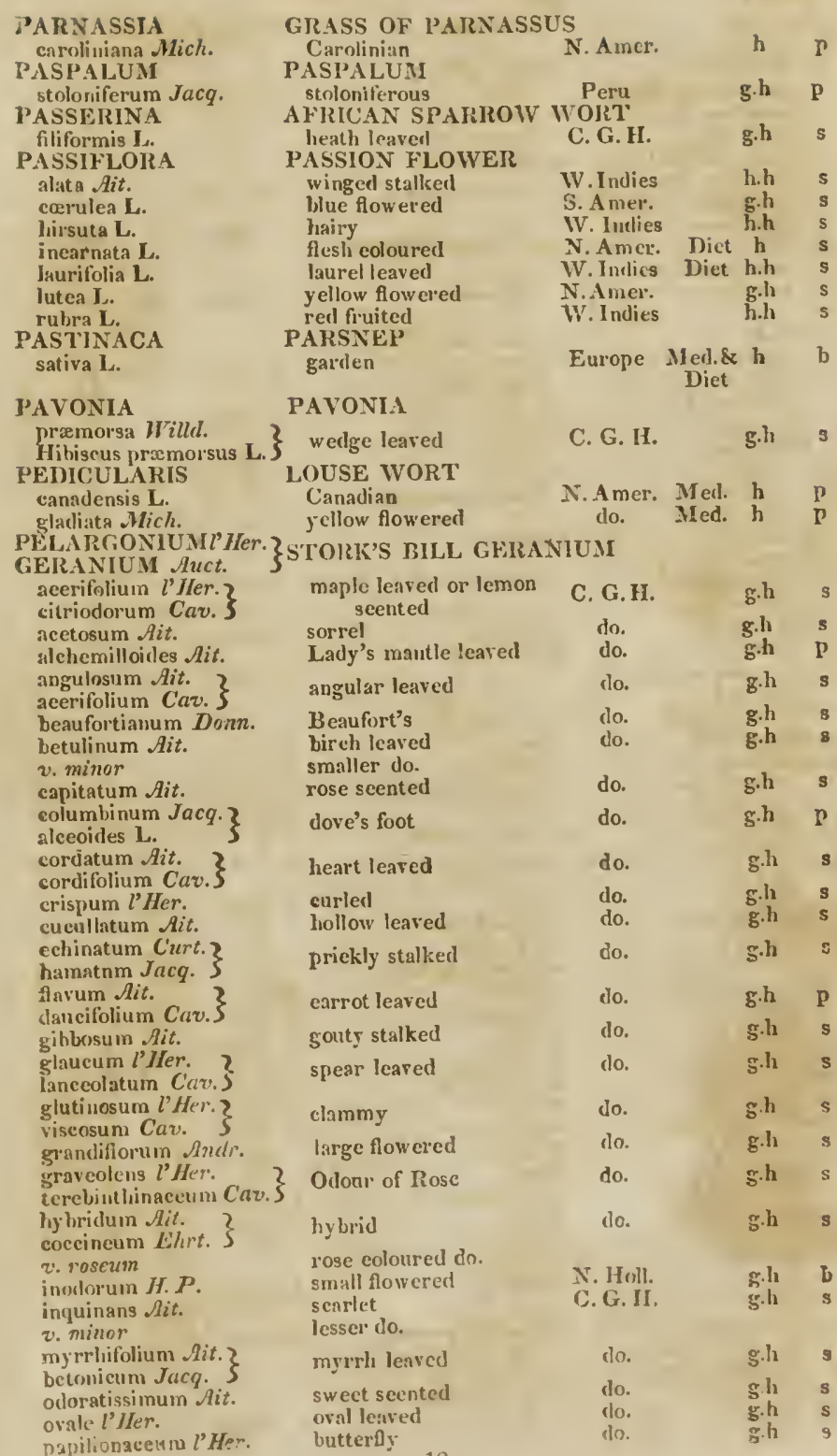


peltatum . Ait.

quercifolium $l$ 'Her.

radula l'Her.

revolutum Jacq. $\}$

v. minus

ข. roseum

scabruin l'Her.

tabulare l'Her.

clongatum Cav. $\}$

tomentosum Jacq.

trieuspidatum $l^{\prime}$ fler.

vitifolium Ait.

zonale Ait.

v. album

ข. roseum

v. marzinatum

PENSTEIION

puivescens Ait.
Chelone penstemon L. puple

PENTA PETES

plicenicen $\mathbf{L}$.

PENTHORUM

sedoides $\mathbf{L}$.

PERIPLOCA

græea 1.

PETIVERIA

alliacea $\mathrm{L}$.

PHALARIS

arundinacea L. Ait. $\}$

v. striata

eauariensis L.

PHASEOLUS

eapensis $T$ lızınb.

caracalla $\mathbf{L}$.

helvolus $\mathbf{L}$.

lathyroides $\mathbf{L}$.

multiflorus IFilld.

coccineus Lmk. $\}$

v. albus

nanus $\mathbf{L}$.

semierectus I.

vilgaris $\mathbf{L}$.

PHILAIDELPHUS

eoronarius $\mathbf{L}$.

v. nanus

inodorus $\mathbf{L}$.

PHILLYREA

angustifolia $\mathbf{L}$.

latifolia L.

merlia $L$.

PHLEUM

norlosum L.

pratcuse $\mathbf{L}$

PHLOMIS

fiuticosa $\mathbf{L}$.

herha venti $L$.

Jconurus $\mathbf{L}$.

jnartinicensis Sruz.?

caribiea Jacy.

purburca Sim.

PIILOX

maculata L.

paniculata L. shield leaved

oak leaved

rasp leaved

lesser do.

rose eoloured do.

rough

rough stalked

downy

three pointed

vine leaved

horse shoe

white do.

rerl do.

variegated leaved do.

PENSTEMON

\section{PENTAPETES \\ scarlet \\ PENTHORUM \\ common \\ PEIRIPIOCA \\ common \\ GUINEA-HYN WEED \\ cornmon \\ CANARY GRASS}

\} reed

ribbon grass

common

KIDNEY BFAN

cape

snail fowererl

deltoid leaved

clickling vetel

searlet runners

Dutch white runners

six weeks

upright

conimon

SYRINGA or Nock Orang

conumou

dwart'

large flowered

PHILLYREA

narrow leaved

broarl leared

privet leaved

CAT'S TAIL GRASS

knotted stalked

mearlow-Timothy

PHLOMIS

Jerusalem Sage

rough leaved

lion's tail

globular leaved

purple

L) Y(H NIDEA

spotted stalkerl

panicled
C. G. 11 .

do.

g.h 8

g.lis

do.

sh s

do.

g.h s

do.

g.l b

do.

do.

do.

do.

g.h s

g.h s

$\begin{array}{ll}\text { g.h } & \text { s } \\ \text { g.h } & \text { s }\end{array}$

N. 1 mer.

h $\quad$ p

E. Indies

h.h b

N. Amer.

h p

Syria

Med. h s

W. Indies

h.h s

Europe

Agr. h P

Can. Isl. Agr. h a

C. G. H.

F. Iudies

N. Amer.

W. Indies

S. Amer.

h.h

h. p

h.h s

Dict h

F. Indies

S. Amel.

E. Indies

Dict h a

Dict $\mathbf{h}$ a

Europe Arts h s

N. Amer. Arts h s

S. Europe $\quad$ g.h $\mathbf{s}$

do. g.li s

do. g.h s

$\begin{array}{ccc}\begin{array}{c}\text { Europe } \\ \text { do. }\end{array} & \mathbf{h} & \mathbf{p} \\ \mathbf{h} & \mathbf{l}\end{array}$

S. Europe Med. g.h s

$\begin{array}{lll}\text { C. G. H. } & \text { gh } & \text { p } \\ \text { G.h } & \text { s }\end{array}$

W. Inties h a

S. Europe $g . h_{\text {s }}$ s

$\begin{array}{ccc}\text { N. Amer. } & h & p \\ \text { do. } & h & p\end{array}$ 
HORTUS ELGINENSIS.

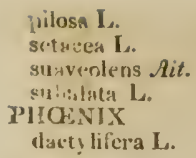

\section{PIIRYMA}

leptostachya L.

PIIVIIC:I

crienialfs $\mathbf{L}$.

piminusa $\mathbf{L}$.

PHKSALIS

alk:kengi L.

angulata L.

inisars $H . P$.

Junsylvarica L.

sutruifera 1.

PHYTOL ICCA

decandra $\mathrm{L}$.

\section{PIC:RIS}

globulifera IX. $P$.

his recinis'es L.

PIMPINELLA

anisam L.

\section{PINUS}

alba Ait.

lax: Elirt.

A bies alba $I I . P$.

balsanea L.

A bies balsainca $I I . P\}$

caurlensis $\mathbf{L}$.
bies canadensis $H, \nu^{\prime}$

incips Ait.

Iarix I.

I.alix europiea $H . P$. $\}$

nigi" Ait.

nariuna Elsrt.

A bies nigra $\boldsymbol{H} . \boldsymbol{P}$.

palustris Ait.

pendula Rit.

pinea L.

resinosa Ait.

rigida Mich.f.

serotina Mich.

strobus L.

PIPER

nigrum $L$.

PISCIDIA

erthrina $L$.

PISUM

maritimum L.

sativum 1.

PIANTAGO

amplexicanlis $C a v$.

crassa Willd.

crispa Lmk. $\}$

lanceolata $\mathbf{L}$.

major $\mathbf{L}$.

maritima $\mathbf{L}$.

maxima Jacq. ?

eucullata Imk.S

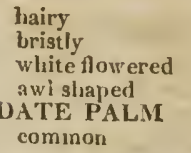

PIIRYMA

sm.ill flowered

PHYLICA

hesth leaved

feathererl

WINTER CIHERRY

common

angetar

hoary

Pennsylvanian

cluster fiowered

POKE WEED

common

OX TONGUE

globulal.

hawk weel

IUURNET SAXIFRAGE A nise

PINE or Fir tree

white or single Spruce

Silver Fir-Balm of Gilead

Iremlock Spruce

Jersey or scrul, Pine

common Larch

black or double Spruce

long leaved or Georgia pitch Pine

black Larch

Italian or stone Pine

pitch Pine

pond Pine

Wevmouth or white

PEPPER

black

PISCIDIA

Coral tree leaved

PEA

sea

common

PLAN'TAIN

stem clasping

thick leaved

Ribwort

great

sea

Iroad or cowl leaved
N. $\Delta$ mer.

do.

do.

do.

Syria Diet \& h.h t

Med.

N.Amer. Med, h p

C. G. H.

s.h s

do.

g.l s

Europe Med. h p

B. Indies h a

N. Amer. Med. g.h J

Mexico g.h s

N. Amer. Diet \& h

Med.

Europe $\quad$ h

Egypt Med. h a

N. Amer. Arts h $t$

do. Med. h

do. Arts h t

$\begin{array}{lll}\text { do. Arts } & h \quad t \\ \text { Europe Arts \& } & h\end{array}$

Med.

N. Amer. Arts h t

do. Arts $h$ t

do. Arts $\mathrm{H}$

S. Europe Diet h

N. Amer. Arts h t

do. Arts h t

ilo. Arts h

B. Indies Med.\& h.h s Dict

S. Amer. h.h s

N. Amer. Diet h p

Europe Diet h :

Europe h a

do. $h \quad p$

N. Amer. Med. h p

do. Med. h p

dlo. h p

Siberia h 
virginica $I$.

PLA'TANUS

occidentalis $\mathrm{L}$.

PLUMBAGO

rosea $L$

PLUMERI

alla $\mathrm{L}$.

rubra L.

POA

angustifolia L.

annua L.

compressa L.

flava $\mathrm{L}$.

lineata Pers. ?

striata Nich. $\}$

pectinacea Mich.

pratensis L.

trivialis $\mathrm{L}$

PODALYIRIA

australis Willd.

Sophora australis $\mathbf{L}$.

tinctoria II illd.

Sopliora tinctoria L.

PODOPHYLLUM

peltatum $\mathbf{L}$.

POLYANTHES

tuberosa L.

POLYGALA

incarnata L.

senega $\mathbf{L}$.

verticillata L.

POLYGONUM

arifolium $\mathbf{L}$.

aviculare $\mathbf{L}$.

bistorta $\mathbf{L}$.

convolvulus L.

fagopyrum $\mathbf{L}$.

hydropiper $\mathbf{L}$.

lipathifolium Ait.

pensvlvanicum Curt. $\}$

minus Ait.

ingustifolium Roth. $\}$

orientale L.

pensylvanicum $\mathrm{I}$.

persicaria L.

ramosissimum .7fich.

sagittatum L.

scandens L.

tenue . Iich.

virginicum $L$.

POLITPODIUM

connectile Mich.

vulgai.e L.

PONTEDERIA

cordata $L$.

POPULUS

alba $\mathbf{L}$.

angulata Ait. ?

angulosa Mich.S

balsamifera $\mathbf{L}$.

dilatata Ait.

fastigiata Mesf

italica Hort.

grandidentata ALicli.
Virginian

N. A mer.

PLANE TREF or Large

Americun

LEAD WORT

red flowered

PLUMERIA

white

red

MEADOW GRASS

narrow leaved

divarf

blue grass

yellow

striped secded

comb flowered

common

field

PODALYRLA or Wild Indigo

Button Wood
N. Amer. Arts h

W.Indies h.h \&

IV. Indies h.h s

do.

h.h s

Europe Agr. h P

N.Amer. Agr. h a

do. Agr. li $\mathrm{P}$

do. Agr. $\mathrm{h}$ p

do. Agr. $h$ p

do. Agr. h a

do. Agr. h p

do. Agle h p

blue flowered

Amer. Arts h p

dyer's do. Arts h l

DUCK'S FOOT or May Apple peltaterl

TUBEROSE

common

MILK WORT

flesh coloured

Seneca snake root

whorlexl leaved

PERSICARIA

spear leaved

Knot grass

Bistort or Snake weed

black bind weed

Buck-wheat

water pepper

pale flowered

small

eastern or tall

l'ennsylvanian

spotted

branched K not grass

srrow leared

climbing

slénder KKnot grass

Virginian

POLYPODY

small flowered

common

PONTEDERIA

heart leaved

POPLAR TREE

European white

angular-Cotton tree

T'acamahac

Italian or Lombardy

Canada
N. Amer. Med. h

E. Indies

g.h P

N.Amer. h a

do. Med. h I

do. $h$ a

do. Merl. h p

N.Amer. Mel. h

Asia Diet h a

N. Amer. Med. h a

do. h a

do. Med. h a

E. Indies h a

N. Amer. Med. h

do. Med. h a

do. h a

do. h a

do. h p

do. h 2

do. h p

N. Amer.
do. Med. h

N.Amer. h p

Europe $h$ t

N. Amer. Arts h t

do. Med. h $t$

Italy h t

N. Amer, Arts h 1
N.Amer, h a

Europe Med. h p 
HORTUS ELC:INTNSIS.

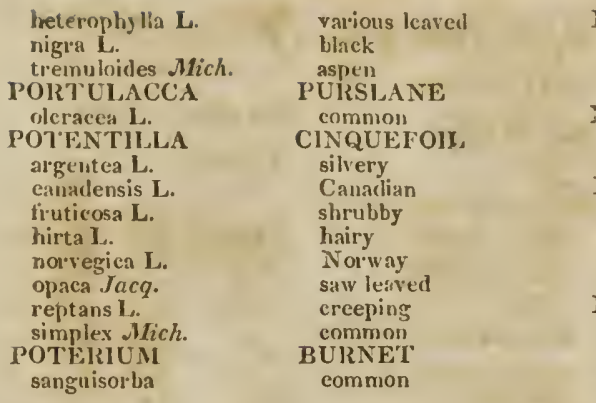

\section{POTHOS}

POTHOS

foeticla Ait. N. Amer. Arts
(lo.
Arts do. Arts li

N.Amer. Diet $h$ a

\begin{tabular}{|c|c|c|}
\hline Europe & & ht \\
\hline $\begin{array}{l}\text { N. Amel. } \\
\text { do. }\end{array}$ & & \\
\hline Europe & & \\
\hline Norway & & \\
\hline Eurnje & & \\
\hline N. Áner: & Med. & \\
\hline & & \\
\hline Europe & $\begin{array}{l}\text { Medl.\& } \\
\text { Diet }\end{array}$ & \\
\hline
\end{tabular}

PIRENANTHES alla $\mathbf{L}$.

altissima $\mathbf{L}$.

PRIMULA

auricula $\mathbf{L}$.

v. lutea

clatior L.

farinosa $\mathbf{L}$.

veris $\mathbf{I}$.

vulgaris $\mathbf{L}$.

acaulis $\mathrm{Sm}$. grandiaora $L m k$.

PRINOS

glabra L.

verticillatus L.

PROSERPINACA

palustris $\mathbf{L}$.

PRO'TEA

argentea $\mathrm{L}$.

scricen 1.

PRUNELLA

pensylvanica Milld. orata $7 . P$.

PRUNUS

al'meniaca $\mathbf{L}$.

avium $\mathbf{L}$.

caroliniana Ait.

cerasus $\mathbf{L}$.

v. flore pleno

cerasifcra Ehrt.

PRENANTHES

wlite flowercil

tall

J'RIMIROSE

Atricula

yellow flowered ilo.

Oxlip

lird's eye

Cowslip or Paigle

eornmon

WINTER BERRY evergicen-Ink berry deciduous

\section{PROSERPINACA}

$$
\text { marsh }
$$

PROTEA

Silver tree

silky

SELF HEAL

common American

CHLRRY TREE

Apricot tiee

small bird

evergreen

commou

double flowered do.

Mijrobalar:

Chickasaw Plumb

common Plumb

common Laurel

black

conmmon bird

upright

dwarf

Anerican Sloe

punila 1.

pygmes Trillil.

semperforens Ehrt. ?

scrotina liotic.

serotina Ellrt.

smharecarpa otich. choak

wild

sca ل"umb
N. Amer. Med. h p

N. Amer. Med. h p do.

Europe

do.

do.

do.

$h$

do. Diet $\mathrm{h}$ p

N. Amer. Arts lis do.

N. Amer.

b b

C. G. II. do.

$\begin{array}{ll}\text { g.h } & \text { t } \\ \text { g.h } & \text { s }\end{array}$

N. Amer. Med. h p

Lerant Diet h $t$

Europe Mesl, h $t$

Carolina Diet $\mathrm{g} \cdot \mathrm{h} \quad \mathrm{t}$

Europe Dict\& h

Med.

N.Amer, Dict \& $h$ t

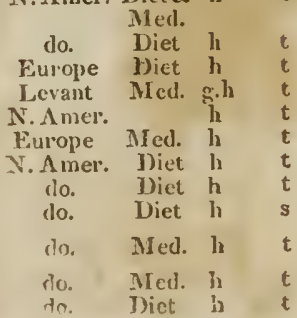




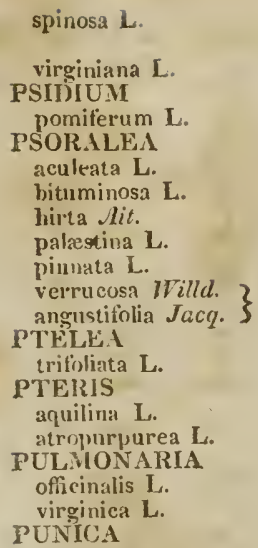

spinosa $\mathbf{L}$.

virginiana $\mathbf{L}$.

PSIIIUM

pomiterum $\mathbf{L}$.

PSORALEA

aculeata $L$.

bituminosa $\mathbf{L}$.

hirta Sit.

palaestina $\mathbf{L}$.

piunata L.

verrucosa Willd.

angustifolia $\mathrm{J} a c q$.

PTELEA

trifoliata L.

PTERIS

aquilina L.

atropurpurea $L$.

PULMONARIA

officinalis $\mathbf{L}$.

virginica $\mathbf{L}$.

PUNICA

grauatum L.

v. fore pleno
PYC NA NTHEMUI

incunun Mich.

Clinopodium incanum L. $\}$ hoary

virginicum Pers.

Thymus virginicus L.

Brachystemum virgi-

PYRETHRUN narrow leaved

FEVER FEW

parthenium Willd.

Matricaria partheni- $\}$ common uns L.

ptarmicæfolium Willd.

Anthemis ptarmicefo- $\}$ snceze wort leaved lia Ilort.

serotinum Willd.

I'Y ROLA

maculata $L$.

rotundifolia $\mathbf{L}$.

umbellata L.

IYIIUS

srbutitolia Willd.

Mespilus at:butifolia L.

Arouia arbutifolia

$$
\text { Pers. }
$$

baccata L.

truited

late flowering

WINTER GREEN

variegated leaved

round leaved

umbeller

I'EAR OR APPLE

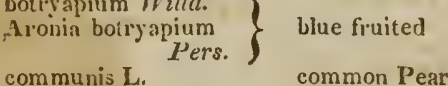

communis $\mathbf{L}$.

coronaria $\mathbf{J}$.

cydonia $\mathbf{L}$.

malus L.

pollveria L.

prunitolia ITillu.

spectabilis Ait.

QUASSIA

amara L. common Pear

sweet scented Crab

common Quince

common Apple

powdered

Lady Apple

Clinese

QUASSIA

bitter
N. Aner.

Med. $\mathbf{b}$

t.

Diet $h$ t

W. Indies Diet h.h t

C. G. H. g.h s

Italy g.h s

C. G. H. g.h 8

Levant gh 8

C.G.H. g.h 8

do. g.h 8

N. Amer. h s

N.Amer. h p

(lo. $h \quad p$

Europe Med. h p

N. Amer. Med. h p

S. Europe Med.\& g.h

Diet

N. Amer. Med. h

do. Med. h p

Europe Med. h D

Tartary h p

N. Amer. h p

N. Amer. Med. h p do. Med. h $p$ do. h $\mathrm{h}$

N. Amer. h p

Siberia Diet $h$ t

N. Amcr. $\quad$ li t

Europe Med.\& li $t$ Diet

N.Amer. Diet $h$ t

Europe Med.\& h $t$

do. Mel.\& $\mathbf{h} t$

$\begin{array}{cccc}\text { do. Diet } & & \\ & \mathbf{h} & \mathbf{t} \\ \text { iberia } & \text { Diet } & \mathbf{h} & \mathbf{t}\end{array}$

China h

Surinam Med. hoh th 
HORTUS ELGINENSIS.

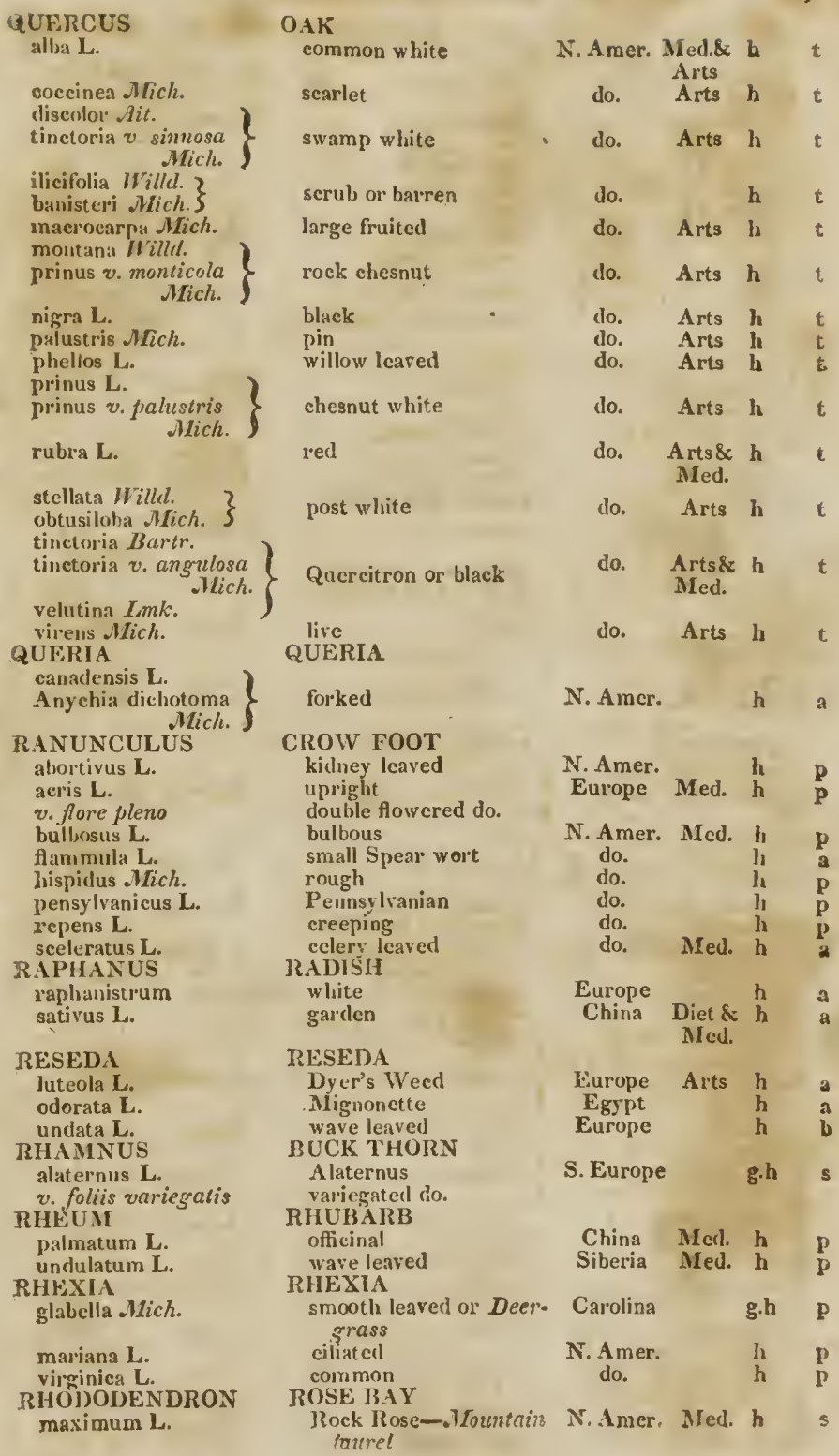


ponticum $\mathbf{L}$.

RIUS

copallinum $\mathbf{I}$.

cotinus $\mathrm{l}$.

glabrum L.

radicans $\mathbf{L}$.

toxinodendron $\mathbf{L}$.

typhinum L.

vernix L.

\section{RIBES \\ grossularia $\mathbf{L}$. nigrum $\mathbf{L}$.}

pensylvanicum $L m k$. ? floridum Willd.

rubrum $\mathbf{L}$.

ข album

uva crispa $\mathbf{L}$.

RICINUS

africanus Willd. ?

communis Desf. $\}$

communis L. viridis Willd.

\section{RIVINA.}

levis $\mathbf{L}$.

ROBINIA

caragana $I_{\text {. }}$

hispida Ait.

pseud-acacia L.

pyginaa L.

viscosa Vent. glutimosa Curt. $\}$

\section{ROSA}

alba $\mathbf{L}$.

bifera Poir.

semperfloreus $\boldsymbol{I I}$. P. $\}$

burgundiaca $H$. $P$.

carolina $\mathbf{I}$.

corymbosa Ehrt. $\}$

centifolia L.

chinensis Jacq.

cinnamomea $\mathbf{L}$.

collincola Fhrt.

majalis Retz.

damascena Ait.

eglinteria L. ?

lutea Ait.

gallica L.

v. versicolor

kainschatica Vent. ?

ferox? Jlonn.

lævigata Mich.

lucida Ehrt.

moschata Ait.

multiflora Thunb.

muscosu Ait.

larviftora Ehrt.

pensylvauica Jitich. $\}$

parvifolia Fhrt. ?

remensis $I . P$.

pimpinellitolia L.

\section{purple \\ SUMACH \\ copal \\ Venice \\ common}

climbing-poison vine poison oak woolly poison ash or Varnish tree

CURRAN'T

common Gooseberry

European black

American black

red

white

smooth Gooseberry

PALMA CHRIS'Ti or Castor Oil plant

\section{African}

coinmon

green

IIVINA

smooth

ROBINIA or Locust tree

Caragana

Rose Acacia

common or False Acacio

dwarf

clammy

ROSE

white

monthly

pompone or burgundly

Carolinian

hundrerl leared

pale Clina

Cinnamon

damask

sirgle yellow

rerl officinal

variegated

Kamschatka

Cherokee

shining leaved

musk

many flowering

moss

Pennsylvanian

Champagne

barnet leaverl
Europe

N. Amer. Arts h s

Europe Arts h s

N. Amer. Arts \& h s

Med.

do. Med. $h$ s

do. $h$ s

do. Arts $h$ s

do. $h$ s

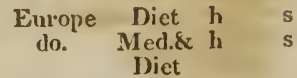

N.Amer. Diet h 8

Europe Med.\& h s

Diet

\begin{tabular}{|c|c|c|}
\hline Africa & & h.h \\
\hline $\begin{array}{l}\text { E. Indies } \\
\text { do. }\end{array}$ & Med. & $\begin{array}{l}\text { h } \\
\text { hi }\end{array}$ \\
\hline WV. Indies & & h.h \\
\hline $\begin{array}{l}\text { Siberia } \\
\text { N. A mer. } \\
\text { do. } \\
\text { Siberia }\end{array}$ & Arts & $\begin{array}{l}\mathbf{h} \\
\mathbf{h} \\
\mathbf{h} \\
\mathbf{h}\end{array}$ \\
\hline N. Amer. & & I \\
\hline
\end{tabular}

Europe Med. h s

do. $h$ s

do. $h$ s

N. Amer. Med. h s

Furope Med. h s

China $\mathbf{s} \cdot \mathrm{h}$ s

Europe $h$ s

Europe Med. h s

do. $h \quad s$

do. Med. h s

Kamschatka h s

Carolina h s

N. Amer. $\quad h$ s

Africa Arts h s

Japan h

Curope h s

N. Amer. Med. h s

S. Europe h s

io. $h$ s 
HORTUS ELGINENSIS.

provincialis Ait.

ruliginosa $\mathbf{L}$.

semperfiorens Curt.

disersifolu Vent.

setiger'a Wich.

spincosissinat.

v. fore pleno

ROSMARINUS

officins lis $\mathbf{L}$.

ROTIIIA

ch(-u)athifolia $\boldsymbol{W}$ illd

$\left.\begin{array}{l}\text { Ardryala sinuat: } \mathbf{L} \\ -1 \text { ciniata } L m k\end{array}\right\}$

\section{RUBUS}

ideus $\mathbf{L}$.

occirlentalis $\mathbf{L}$.

orlon'atus L.

sax: rilis L. ?

v. americana $S$

strigosus $\boldsymbol{N i c h}$.

trivialis $\boldsymbol{A i c h}$.

$\left.\begin{array}{l}\text { villnsus Ait. } \\ \text { vulpinus Ilesf }\end{array}\right\}$

RUIDECKIA

an!rilexifolis Jacq. ?

perfoliat: $C u v$.

laciniaty $\mathbf{L}$.

purpurea $\mathrm{L}$.

RUM!F:X

acetosa L.

acetosella L.

acutus L.

alpinus $\mathbf{L}$.

crispatulus Mich. 3 persichrinirles

xanthoriz: Pers. ?

brittaniea L.

\section{RUSCUS}

aculeatus $\mathbf{L}$.

atudrogy nus $\mathbf{L}$.

RU'TA

graveolens $\mathbf{L}$.

SABAL

niinor Pers.

Cory pha ninor Jacq.

Cheiniserops acaulis

SACCIIARUM officinatun $L$.

SA BINA

procunilieus L.

SAGITTAIRA

lanciorilia L.

latilolia Willd.

obtusa .Mulul. 2 simplex Pers

SALICURNIA

virginica L.
Provence

swcet briar

red China

bristly

Scoteh

doubl. Anowered do.

ROSFMARY

conimon

IRO'THIA

stock leaved

BRA MBLE

garden liaspluerry

American Raspberry

flowcring Raspberry

small American

mountain

American Dewberry

A merican Blackberry

RUDBECKIA

stem clasping

cut leaved

purple flowcred

DOC: $\mathrm{K}$

garden Sorrel

shcep Sorrel

sharp pointed

Monk's Rhuharb

common Ancrican

bloody

jellow rooted

BU'TCHER'S BROOM

prickly

climbing

IRTE:

common

DUVARF PALM

fan

SUGAR CANE

commen

PEARL, WORT

pucumlier:

ARRUIT HHAD

spear leaver

broad leaved

simple stalked

GL.ISS IVORT

common S:ıniphire

14
S. Furope

N. A mer.

China

N. Amer.

Europe

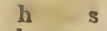

h s

g.h s

S.Europe Med. g.h s

Europe b a

Europe Med.\& h s

Diet

N.Amer. Ditt \& h s

Med.

do. h s

do. h s

do. h $\mathrm{s}$

do. Diet \& h s

Merl.

do. Diet \& h s

Med.

S. Amer.

h a

N. A mer.

$\begin{array}{ll}\text { h } & \text { p } \\ \text { h } & \mathrm{p}\end{array}$

do.

Europe Diet \& h p

Med.

N. Amer. Dict\& h p

Med.

do. h $p$

Europe Med. h p

N. Amer. h

do. h $\mathrm{p}$

do. h p

Europe Med. h s

Can. Isl. , g.h s

Europe Med. h s

Carolina Arts g.h s

B. Indies Diet h.h

Europe h b

N.Amer.

do. b p

do. h $\mathrm{l}$

N. Amer. Diet h a 
HORTUS ELGINENSIS.

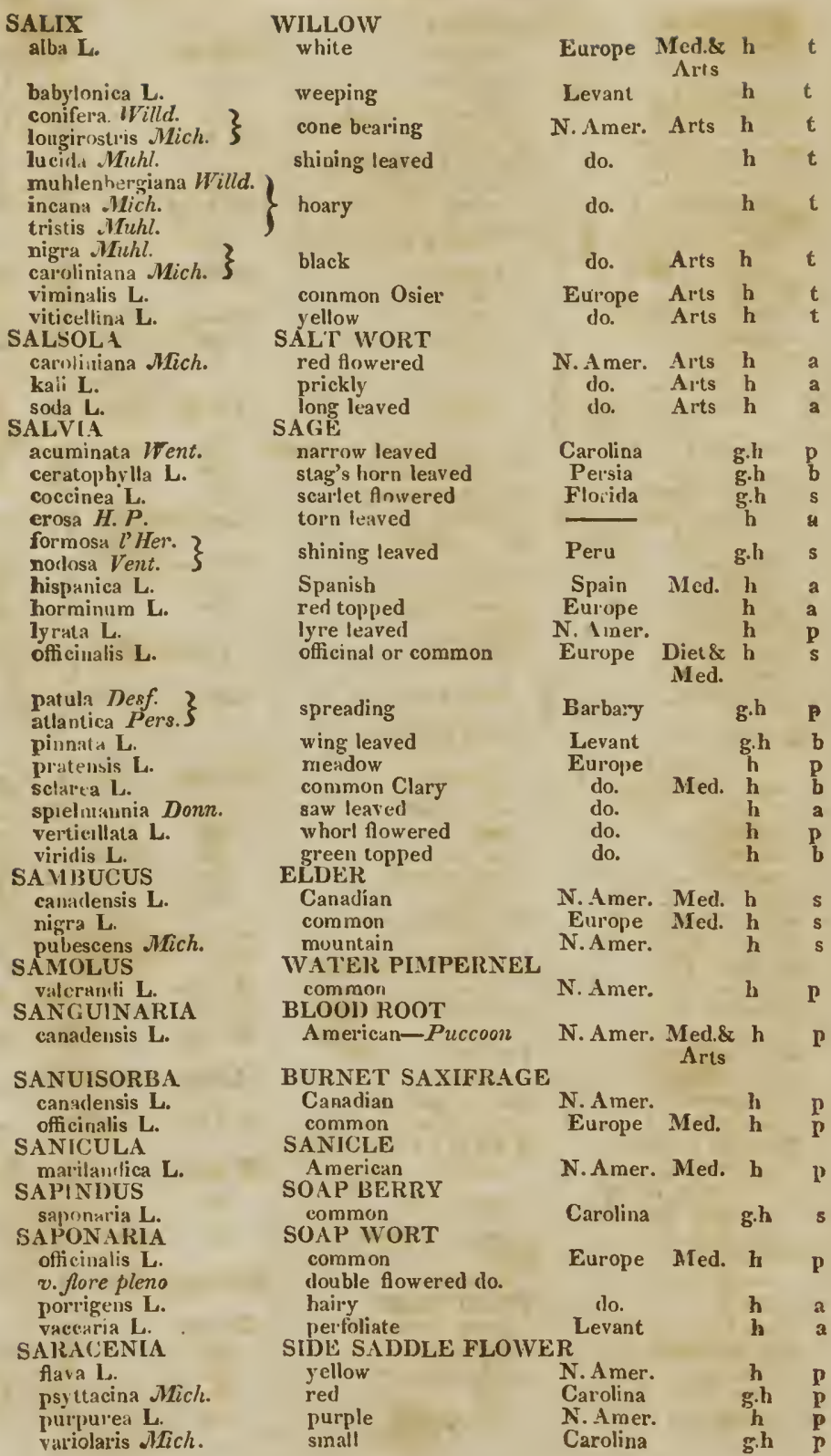


HORTUS ELGINENSIS.

\begin{tabular}{|c|c|c|c|c|c|}
\hline & & & & & \\
\hline lortensis $\mathrm{L}$. & summer & Europe $N$ & $\begin{array}{l}\text { Med.\& } \\
\text { Diel }\end{array}$ & h & \\
\hline montuna & winter & do. & Diet & $\mathbf{h}$ & \\
\hline $\begin{array}{l}\text { SAUt } \\
\text { cern }\end{array}$ & $\begin{array}{l}\text { LIZA RID'S TAIL } \\
\text { nodding }\end{array}$ & N. Amer. & & & \\
\hline $\begin{array}{l}\text { SAXI } \\
\text { crus }\end{array}$ & $\begin{array}{l}\text { SAXIFRAGE } \\
\text { thick leaved }\end{array}$ & & & & \\
\hline L. $\mathbf{L}$. & merican & N. Amer. & & $\stackrel{\mathbf{n}}{\mathbf{h}}$ & \\
\hline $\begin{array}{l}\text { sarmentusa Ait. } \\
\text { stuloniler: Jacq. }\end{array}$ & creeping or strawberry & China & & g.h & \\
\hline $\begin{array}{l}\text { virguica Mich. } \\
\text { SCABIOS. }\end{array}$ & $\begin{array}{l}\text { Virginian } \\
\text { SCABIOUS }\end{array}$ & N. Amer. & & h & \\
\hline $\begin{array}{l}\text { at } \\
\text { atr } \\
\text { ste }\end{array}$ & $\begin{array}{l}\text { sweet } \\
\text { cat's eye }\end{array}$ & Europe I & Med. & h & \\
\hline SCAI & CICEI & & & & \\
\hline $\left.\begin{array}{c}\text { cerefolium L. } \\
\text { Cliaphyllum sati- } \\
\text { vum Pers. }\end{array}\right\}$ & Chervil & Europe & Diet & h & a \\
\hline $\begin{array}{l}\text { odlorata } \mathbf{L} \text {. } \\
\text { pectens L. }\end{array}$ & $\begin{array}{l}\text { scented or Myrrh } \\
\text { s comb }\end{array}$ & $\begin{array}{l}\text { do. } \\
\text { do. }\end{array}$ & Med. & $\begin{array}{l}\mathbf{h} \\
\mathbf{h}\end{array}$ & $\stackrel{q}{p}$ \\
\hline $\begin{array}{l}\text { TDRA } \\
\text { Mich. }\end{array}$ & $\begin{array}{l}\text { SCHISANDRA } \\
\text { scarlet }\end{array}$ & Carolina & & g.b & s \\
\hline $\begin{array}{l}\text { SCHCENUS } \\
\text { albus L. } \\
\text { fuscns } \mathrm{L} \text {. } \\
\text { glomeratus } \mathrm{L} \text {. }\end{array}$ & $\begin{array}{l}\text { BOG RUSH } \\
\text { white } \\
\text { brown } \\
\text { glomerated } \\
\text { SoUllL }\end{array}$ & $\begin{array}{l}\text { N. Amer. } \\
\text { do. } \\
\text { do. }\end{array}$ & & $\begin{array}{l}\mathbf{h} \\
\mathbf{h} \\
\mathbf{h}\end{array}$ & p \\
\hline $\begin{array}{l}\text { SCIILLA } \\
\text { matitima L. } \\
\text { nutans Sm. }\end{array}$ & $\begin{array}{l}\text { SQUILL } \\
\text { officinal }\end{array}$ & S. Europe & Med. $\varepsilon$ & g.h & $\mathbf{p}$ \\
\hline $\left.\begin{array}{r}\text { Hyanthus non serip } \\
\text { tus L. }\end{array}\right\}$ & IIare bell & Europe & Arts & h & D \\
\hline $\left.\begin{array}{l}\text { fCIRl'US } \\
\text { amerieanus Pers } \\
\text { triqueter Wich. }\end{array}\right\}$ & $\begin{array}{l}\text { CLUB RUSH } \\
\text { three sided }\end{array}$ & N. Amer. & Arts & h & 1 \\
\hline $\begin{array}{l}\text { capillaris .Mich. } \\
\text { capitatus L. }\end{array}$ & $\begin{array}{l}\text { lhair shaped } \\
\text { headed }\end{array}$ & do. & & h & a \\
\hline $\begin{array}{l}\text { lacus } \\
\text { mact }\end{array}$ & & $\begin{array}{l}\text { do. } \\
\text { do. }\end{array}$ & $\begin{array}{l}\text { Arts } \\
\text { Arts }\end{array}$ & $\begin{array}{l}\mathbf{h} \\
\mathbf{h}\end{array}$ & \\
\hline $\begin{array}{l}\text { mace } \\
\text { sylv: } \\
\text { SCLLE }\end{array}$ & & & & & \\
\hline $\begin{array}{l}\text { NTHUS } \\
\text { US }\end{array}$ & TIISTLE & N. Amer. & & $\mathbf{h}$ & $\mathbf{a}$ \\
\hline $\begin{array}{l}\text { SCO } \\
\text { hi } \\
\text { SCO }\end{array}$ & ILLARS & Europe & & h & p \\
\hline $\begin{array}{l}\mathrm{SCC} \\
\mathrm{SCC}\end{array}$ & GRASS & Europe & & h & $\mathbf{a}$ \\
\hline $\begin{array}{l}\text { SCO } \\
\text { lis } \\
\text { SClR }\end{array}$ & $\begin{array}{l}\text { cominon } \\
\text { FIG WURT }\end{array}$ & Europe & Diet & $\mathrm{n}$ & p \\
\hline $\begin{array}{l}\mathrm{SCl} \\
\mathrm{m}: \\
\mathrm{SCU}\end{array}$ & ScUl & N. Amer. & d. & h & $\rho$ \\
\hline SC & eaved & N. Amer. & & $\mathbf{h}$ & p \\
\hline $\begin{array}{l}\text { int grifolia L. } \\
\text { ovulifolia Pers. } \\
\text { ateriflora L. }\end{array}$ & oval leaved & $\begin{array}{l}\text { do. } \\
\text { do. }\end{array}$ & & & $P$ \\
\hline $\begin{array}{l}\text { SEC.ALE } \\
\text { certeale L. }\end{array}$ & $\begin{array}{l}\text { RYE } \\
\text { STO }\end{array}$ & S. Europe & Diet & h & a \\
\hline $\begin{array}{l}\text { SEDUM } \\
\text { anacampseros L. } \\
\text { populifolium Ait. } \\
\text { reflexum } \\
\text { rupestie L. } \\
\text { srrangulare L. }\end{array}$ & $\begin{array}{l}\text { STONE CROP } \\
\text { evergreen Orpine } \\
\text { poplar leaved } \\
\text { reflexed leaved } \\
\text { rock } \\
\text { six angled }\end{array}$ & $\begin{array}{l}\text { Europe } \\
\text { do. } \\
\text { do. } \\
\text { do. } \\
\text { do. }\end{array}$ & & $\begin{array}{l}h \\
h \\
h \\
\text { h } \\
\text { h }\end{array}$ & 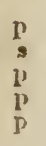 \\
\hline
\end{tabular}


telephium L. ternatum Mich.

SEMPFRVIVUM arlroreum L. tectorum L.

SENECIO

doria $\mathbf{L}$.

elegaus L.

halimifolius L.

sessilis Thunb.

hieracifolius $\mathbf{L}$.

vulgaris $\mathbf{L}$.

SFRISSA

foetida Willd.

Lycium fætidum L

$\left.\begin{array}{l}\text { Lycium fotidum L. } \\ \text { v. fore pleno }\end{array}\right\}$

SESA MUM

orientale L.

SHER ARDIA

arvensis $\mathbf{L}$.

SICYOS

angulata $\mathbf{L}$.

SIDA

abutilon $\mathbf{L}$.

alnifolia $\mathbf{L}$.

angustifolia L. $\}$

innitolia Retz. $\}$

asiatica L.

A nocla triloba Cav. $\}$

incliea $\mathbf{L}$.

populifolia Cav. ?

iseloere l'Her.

rhombifolia $\mathbf{L}$.

spinosa L.

triloha Cav.

SIJEEROXYLUM

lanuginosum Mich.

Bumelia lanuginosa

SILENE

armeria $\mathrm{L}$.

antirrhina $\mathbf{L}$.

conica L.

dichotoma Ehrt.

fruticosa $\mathbf{L}$.

mutabilis $\mathbf{L}$.

nocturna $\mathbf{L}$.

ornata Ait.

pensylvanica Mich.

picta $H . P$.

quinqueruinera $L$.

SILPHIUM

laciniatum $\mathrm{L}$.

SINAPIS

alb. I.

nigz: L.

SIPHON ANTHUS indica 1 .

Pers. common Orpine

three leaved

HOUSELEEK

tree

common

GROUNDSEL

broad leaved

purple Jacobæa

halimus leaved

haw $\mathbf{k}$ weed leaved

common

BOX THORN

Japanese

double flowered do.

SESA MUM

oriental-Bhenne

FIELD MADDER

corn

Europe
SINGLE SHEDED CUCUMBER

angular leaved

SIDA

round leaved

alcler leaved

narrow leaved

small flowered

blue flowered

Indian

poplar leaved

rhomboid leaved

prickly

thiee lobed

IRON IVOOD

\}

\section{CATCHFLY}

Lobel's

snapdragon

corn

Uranched

shrubloy

changeable

night smellins

dark flowering

Penusylvanian

striped flowered

spotted

SILPHIUM

jagged leaved

MUSTARD

white

Ulack

TUBE FLOIVER

Indian
Europe

N. A mer.

Med. $\begin{array}{ll}\text { h } & \text { p } \\ \text { h } & \text { p }\end{array}$

Levant

Europe

Europe

C. G. H.

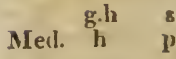

do.

h p

N. Amer. Med.

Europe Merl. h a

Japan $\quad$ g.h s

E. Indies Diet\& h Med.

N. Amer.

Europe

E. Indies

do.

do.

Mexico

E. Indies

do.

do.

do.

do.

h a

h a

h b

a

h a

h a

h a

h a

h a

h.h $\mathrm{s}$

h a

Carolina

h $t$

Europe

N. Amer.

Europe

Hungary

Sicily

Europe

Europe

C. G. H.

N. Amer

Europe

N. Amer.

Europe 1)iet \& h a

Merl.

do. Diet \& h a

Med.

F. Indics

h.h $\mathrm{s}$ 


\section{SISON}

canadense $\mathbf{L}$

Chreropliyllum cana- $\}$ Canadian

trifoliatum L.

SISY MBRIUN

amphilium $\mathbf{L}$.

nasturtium $\mathbf{L}$.

soplia $\mathbf{L}$.

\section{SISYIINCIIUMI}

anceps $l \mathrm{lmk}$.

gramincuin Curt $\}$

mucronatum Mich.

SIUM

falcaria L.

lineare Nich.

rigidius $\mathbf{L}$.

sisarum L.

SMIL. $X$

cordifolia Ifumb.

herbacea $\mathbf{L}$.

laurifolia $\mathbf{L}$.

quadrangularis Muhl.

rotundifolia $\mathbf{L}$

perfuncularis $\mathrm{M} u h l$. ?

pulverulenta Mich. S

sarsaparilla L. 3

glauca Mich. $\}$

sy philitic: Humb.

\section{SNIYRNIUM}

corclatum Nich.

\section{SOLANUM}

clulcamara L.

insanum L.

Jycopersicum $\mathbf{L}$.

jnammosum $\mathbf{L}$.

melongena L.

nigrum L. ?

v. virginicum

pseuclo capsicum $\mathbf{I}$, tuberosum $\mathbf{L}$.

SOLIDACIO

altissim: L.

bicolor $\mathbf{L}$.

eavadensis $\mathbf{L}$.

Aexicaulis $\mathbf{L}$.

Jxvigata Ait.

Chrysocoma gramini-

lateriflora Ait. folia L.

nemoralis Ait.

noveboraccnsis $\mathbf{L}$.

odora Ait.

procera git.

reflexa Ait.

senupervilens $\mathbf{L}$.

\section{SONCHUS}

oleraceus $\mathbf{L}$.

pillidus II illd.

Lactucu canadensis L.

palustris 1.
HONE WORT

three leared

SISYMBRIUM

WVater Radish

Water Cress

Flix weed

SISYRINCHIUMI

grass leaved

blue eyed grass

WATER PARSNEP

sickle leaved

linear leaved

stift stalked

Skirrets

SMILAX

heart leaved

herbacens

laurel leaved

square staked

round leaved

pow dezed

medicinal

true Sarsaparilla

ALEXANDERS

heart leaved

NIGHTSHADE

Bitter Sweet

large prickly Ego plant

Love apple-I'omatoes

smaller prickly Egg plant

common Eggr plaut

common

Jerusalem Chery common Potutoe

GOLDFN ROD

tall

two coloured

Cansdian

bent stalked

smooth leared

spear leaved

lateral flowered

wood

New-York

swect scented

large

reflex leaved

narrow lased

SOWV THISTLF:

common

pale

mar'sh
N. Amer.

h p

do. Med. $\mathrm{b}$ p

N. Amer. Med. h P do. Diet \& $h$ p

Med.

do. Med. h a

N. Amer. $\quad h \quad p$

do. $h \quad p$

Europe $\quad$ h p

N. Amer. $\quad h \quad p$

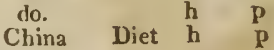

Mexico g.h s

N. Amer. h p

do. $h$ s

do. h s

do. h s

do. h p

do. Med. h s

S.Amer. Med. h.h s

N.Amer. Med. h p

N. Amer, Med. h s

E. Inclies li a

S.Amer. Diet h a

do. Diet $h$ a

do. Diet h a

N. Amer. Med. h a

Madeira g.h $s$

Peru Diet h p

N.Amer. h p

io. h $p$

do. h p

do. h p

do. $\mathrm{h} \quad \mathrm{E}$

do. h p

तo. li $\mathrm{P}$

ho. h th

do. h p

do. h P

do. h $\mathrm{P}$

$\begin{array}{lll}\text { do. } & \text { h } \\ \text { do. } & \text { h }\end{array}$

N. Amer. Med. h a

$\begin{array}{lll}\text { do. } & \text { h } & \text { P } \\ \text { do. } & \text { l) } & \text { P }\end{array}$


HORTUS ELGNENSIS.

\section{SOPHORA}

japonica $\mathbf{L}$.

microphylla Ait. ?

tetraptera L.

tomentosa $\mathbf{L}$.

SORGHUM Pers. ?

HOLCUS Auct.

bicolor Pers.

saccharatum Pers.

spicntum Pers.

vulgare Pers.

\section{SORBUS}

aucuparia L.

canadensis Donn.

SPARGANIUM simplex Sm.

SPARMANNIA afiricana $L$.

SPAR'TIUM

junceum L. $\mathbf{L}$. Desf. $\}$

linifolium Desf.

Genista linifolia L. $\}$

monospernum L.

multiflorum l'Her.

album Desf.

Genista alba Lmk.

scoparium L.

SPERGULA

arvensis $L$.

SPIGELIA

marilandica $\mathbf{L}$.

SPILANTHUS

oleraceus $\mathbf{L}$.

SPINACIA

oleracea $\mathbf{L}$.

\section{SPIR IEA}

crenata L.

hypericifolia $\mathbf{L}$.

opulifolia L.

salicifolia $\mathbf{L}$.

tomentosa L.

trifoliata $\mathbf{L}$.

Gillenia trifoliata

SPONDIAS Monch.

dulcis Forst.

cytherea $\operatorname{Lmk}$. $\}$

STACHYS

annua $\mathbf{L}$.

aspera Mich. tenuifolia Muhl. $\}$

lansta L.

ST EHELINA

chamæjuce $\mathbf{L}$.

STAPFLIA

aperta Willd.

lirsuta L.

variegata L.

STAPHYLEA

trifolia $\mathrm{L}$

STATICE

a:meria L.
SOPHORA

\section{Japan}

small leaved

silvery

араи

s.h s

two coloured

common

oval spiked

cultivated

SERVICE TREE

Mountain Ash

Canarlian

BUlRR REED

American

SPARMANNIA

maple leaved

BROOMF

Spanish

flax leaved

single seeded

white Portugal

common

SPURIREY

corn

SPIGELIA or Worm grass

Carolina Pink Root

SPILANTHUS

eatable

SPINACH

common

SPIREA

crenated

St. John's wort leaved

Snowball leaved

willow leaved

woolly

Bow man's root

Indian Physic-Ipecrcuanha

HOG PLUM

Otaheite Goosberry

HEDGE NETTLE

annual

rough leaved

woolly

ST RHELINA

narrow leaved

STA PELIA

open flowered

hairy

variegated

BL IDDER NUT

three leaved

THRIFT

coramon
S. Sea Isl.

g.h s

E. Indies

h.h s

\begin{tabular}{|c|c|}
\hline Persia & Arts \\
\hline E. Indies & Diet \\
\hline do. & Arts \\
\hline
\end{tabular}

Europe Med. h t

N. Amer. $\quad$ h t

N. Amer. h p

C. G. H. g.h s

Europe Arts h s

Spain g.h s

do.

Portugal h s

Europe Med. h s

N. Amer. h a

N. Amer. Med. h p

E. Indies Diet g.h b

E. Indies Diet \& h $\quad$ h Med.

$\begin{array}{lll}\text { N. Amer. } & \mathbf{h} & \mathbf{p} \\ \text { do. } & \mathbf{h} & \mathbf{s} \\ \text { do. } & \mathbf{h} & \mathbf{s} \\ \text { do. } & \mathbf{h} & \mathbf{s} \\ \text { do. } & \mathbf{h} & \mathbf{s}\end{array}$

do. Med. h p

S. Sea Isl. Diet h.h s

Europe h a

N. Amer. h p

Siberia b p

S. Europe g.h s

C. G. H. h.h 8

do. h.h s

do. h.h s

N. Amer. h s

Europe h p 
limoniurn I.

sinuata 1.

STEWARTIA

malacodendron

virginica Cav.

STILLINGIA

sebifera Willd.
Crnton sebiferum L.

ST'IPA

avenacea $L$.

STRELIT'ZIA

regine Ait.

Heliconia alba L. $\}$

STYIRAX

granclifolium Ait. ?

officinale Walt.

lsvigatum alit. ancricanum $I m k$.

SYMPHITUM oflicinale $\mathbf{L}$.

SYRINGA

chinensis Willd. ?

dubia Pers.

persica L.

v. laciniata

vulgaris $L$.

v. alba

TAGETES

erecta $\mathbf{L}$.

lucida Cav.

patula L.

TAMARINDUS

indicus $\mathbf{L}$.

TAMARIX

germanica $\mathbf{L}$.

TẢ NACE'TUM vulgare 1.

TEPHROSIA

toxicaria Pers.
Galega toxicaria Swo.

TEUC] IUM

campanulatum $\mathbf{L}_{\text {. }}$

canadense $\mathbf{L}$.

fruticans $\mathbf{L}$.

v. latifolium

massilense $\mathbf{L}$.

seororlonia L.

THALIA

lealbata Donn.

THALIC'T RUM

glaucun II. $P$.

lisevigatum Mich.

dioieum $\mathbf{L}$.

polygamuin Mrihl.

purpurascens $\mathbf{L}$.

rugosum Ait.

THEA

bohea Is.

viridis ${ }^{\prime}$.
Sea Laventer-Marsh $\mathbf{N}$. Amer. Med. h Rosemary

wing stemmed

STEIVARTIA.

Virginian

Levant

g.h $\mathrm{p}$

N. Amer.

h s

STILLINGIA

Tallow tree

FEATHER GRASS

oat

STRELITZIA

oval leared

STIRAX

bay leaved

smooth leaved

COMFREY

officinal

LILAC

Chinese

Persian

laciniated do.

common

wlite do.

TAGETES

African Marygold

shining

French Marygold

TAMARIND TREE common

TAMARISK

German

TANSEY

common

TEPHROSLA

Fish-poisou

GERMANDER

small flowered

nettle lenved

shrubbv

broad leaved ito.

sweet scented

wood sage

THALIA

white

MEADOW RUE glaucous

sinooth leaved

tall

purple

rough leaved

TEA TRLE

bohea

grenp

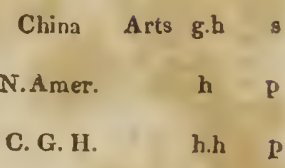

N. Amer. Med, h $t$

do.

h t

Europe MIed. h D

$\begin{array}{ccc}\text { China } & \text { h } & \text { s } \\ \text { Persia } & \text { h } & \text { s } \\ \text { do. } & \text { h } & \text { s }\end{array}$

Mexico Diet la a do. $h$

do. Arts $h$ a

B. Indies Diet \& h.h t Med.

Europe Med. h s

Europe Med. h P

W. Indies

h.h.

Levant

S. Europe N.h

do. $\mathrm{g} \cdot \mathrm{h}$

Europe Med. h $^{\text {p }}$

Carolina s.h P

\begin{tabular}{ccc}
\hline N. Amer. & h & p \\
do. & h & p \\
lo. & h & p \\
do. & h & p \\
& h & p
\end{tabular}

China Med.\& $\mathrm{g} . \mathrm{h}$ s

do. Med.\& g.h s Diet
N. Amer. Med. li b 


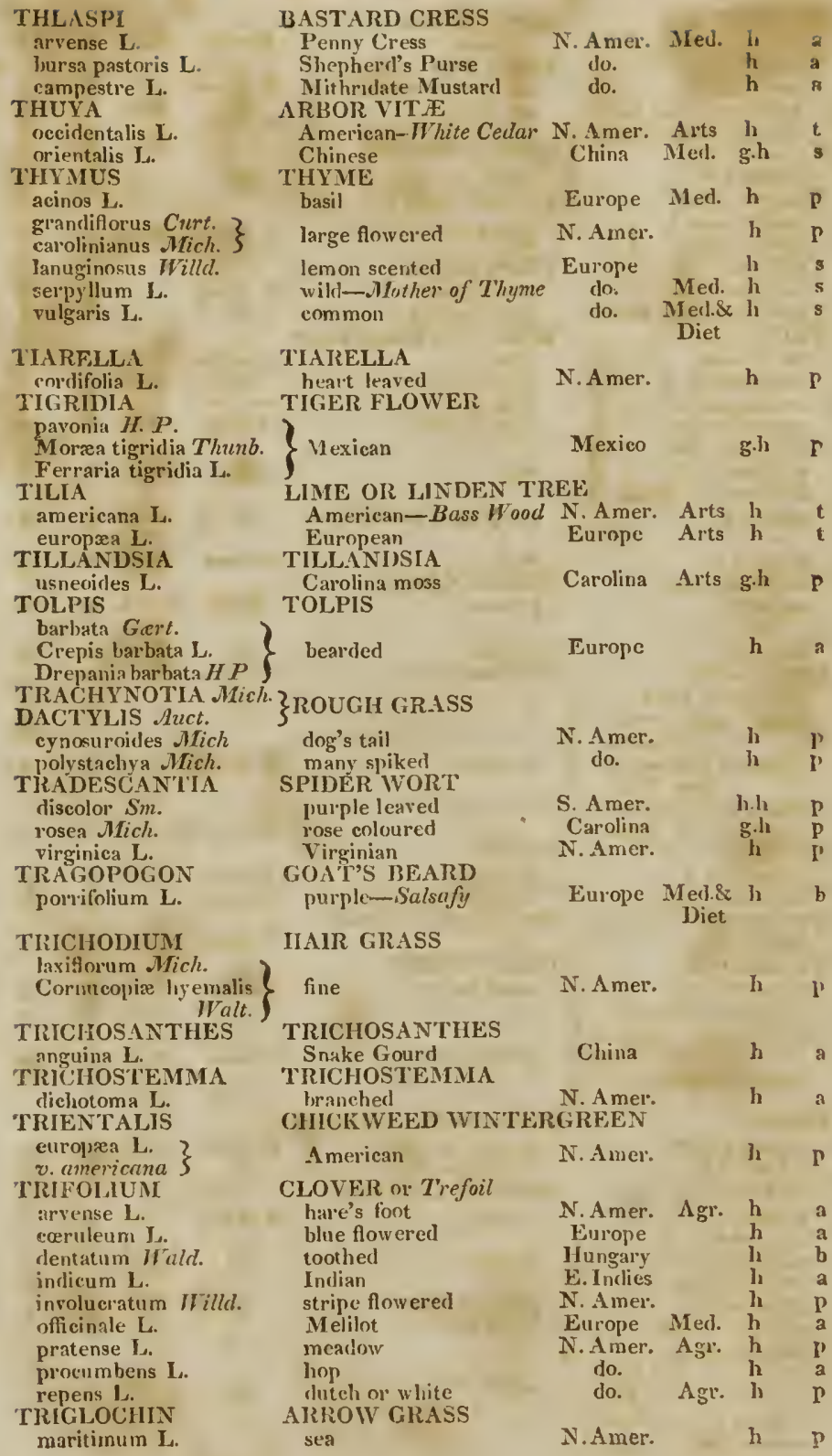


HORTUS ELGINENSIS.

TRIGONELLA

fanum gracum $\mathbf{L}$.

TRILLIU M

cernuum L.

ery throcarpum Mich.

rhomboideum Mich. 2

erectum $\mathbf{L}$.

sessile L.

TRIOS'TEUM

perfoliatun L. ?

majus Mich.

TRIPSAC,UM

dacty loides $\mathbf{L}$.

monostachyon Willd.

TRITICUM

restivum L.

compositum L.

hy bernum $L$.

junceum Sm.

loliaceum Sm.

wilaterale Ait.

Poa Ioliacea Huds.

repens $\mathbf{L}$.

spelta L.

TROPAOLUM

majus $\mathbf{L}$.

minus L.

TROXIMON

virginicum Pers.

Tragopogon virgini-

Hyoseris cum $\mathbf{L}$.

yoseris prenantho: des Willd. lis Mich.

\section{TULIPA}

gesneriana $\mathbf{L}$.

suaveolens Roth.

TURRITIS

liirsuta L.

TUSSILACO

farfara 1.

integrifolia $\mathrm{Mich}$.

Perticium semifloscu-

Chaptalia tomentosa

$$
\text { lare } W a
$$

TYPHA

angustifolia L.

latifolia L.

VACCINIUM

allum $\mathbf{L}$.

col'ymbosum L.

amcenum Ait.

disomorphum Mich. $\}$

frondosum $\mathbf{L}$

glaucum $l, m k$.

Vent.

hispidulum L.
Oxycoccus hispirlulus
small fruited American

Arbutus thymifolia Ait. filiform is $L \mathrm{ml}$
FENUGREEK

common

TIRILLIUM

dronping flowered

red fiuited

upright flowered

sessile flowered

FEVER WORT

perfoliate

TRIPSACUM

three spiked

single spiked

WHEAT or Wheat Grass

summer

Egyptian

winter

rush leaved

sea

creeping-Couch Grass

Spelt

INDIAN CRESS

greater

smaller

TROXIMON

Virginian

TULrP

garden

sweet or Van $\mathrm{Tol}$

TOWER MUSTARD

hairy

COL'T'S FOOT

common

entire leaved

CAT'S TAL or Reed Mace

narrow leaved

bjoad leaved

WHORTLE BERSY

white

Bilberry

obtuse leaved

glaucous

$\begin{array}{llll}\begin{array}{l}\text { Levant } \\ \text { Europe }\end{array} & \text { h } & \text { p } \\ \text { Europe Diet } & \text { h } & \text { b } \\ \text { Europe Med. } & \text { h } & \text { p }\end{array}$

Carolina Med. g.h D

N. Amer. Arts h do. Arts $h$ p

N. Amer. Diet h s

do. Diet \& h s

Med.

do. Diet h $s$

do. Diet h s

do. Diet\& $\mathrm{h}$ s

Med. 


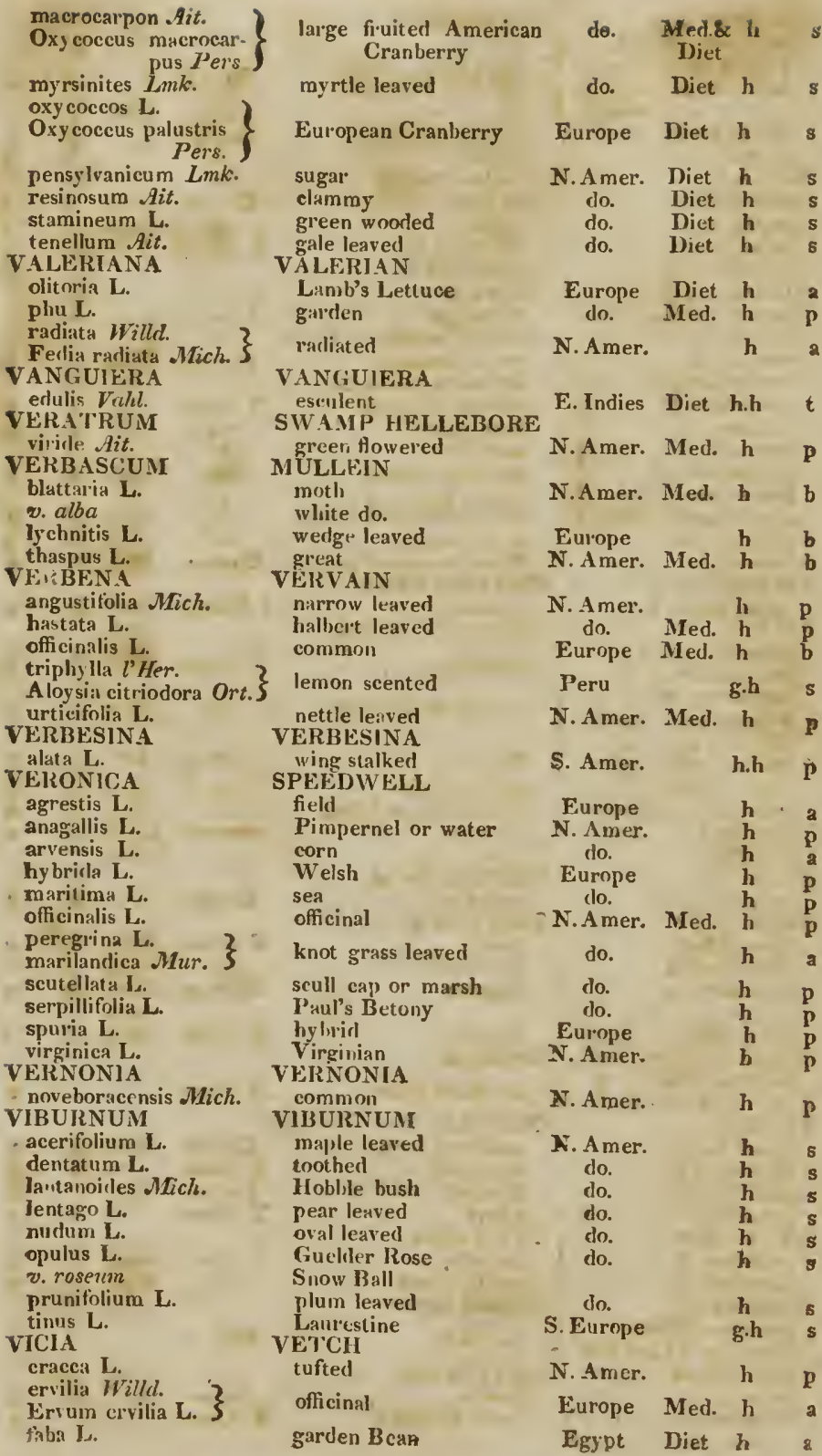




\section{HORTUS ELGINENSIS.}

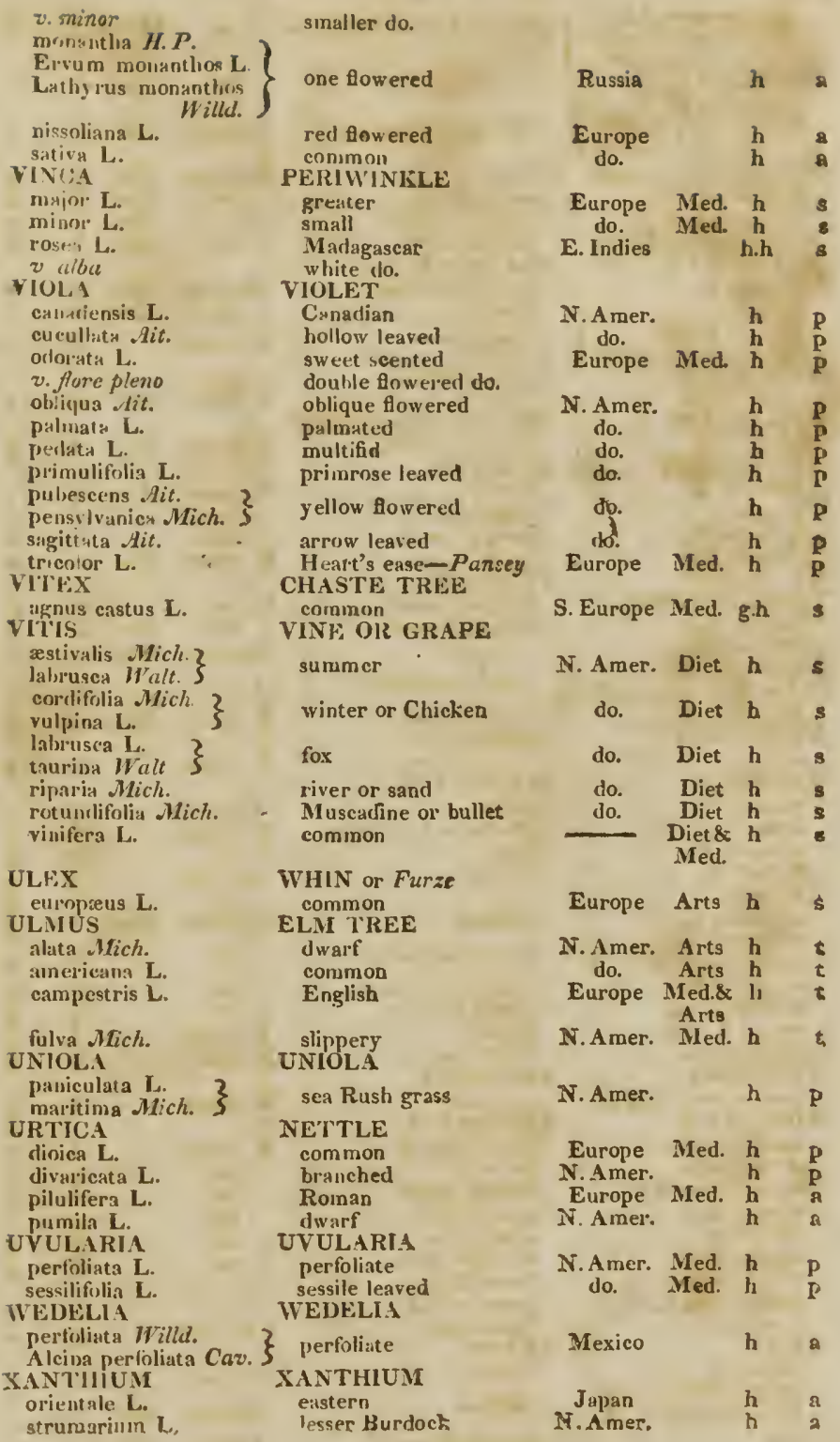


HITORUS ELGINLNSIS:

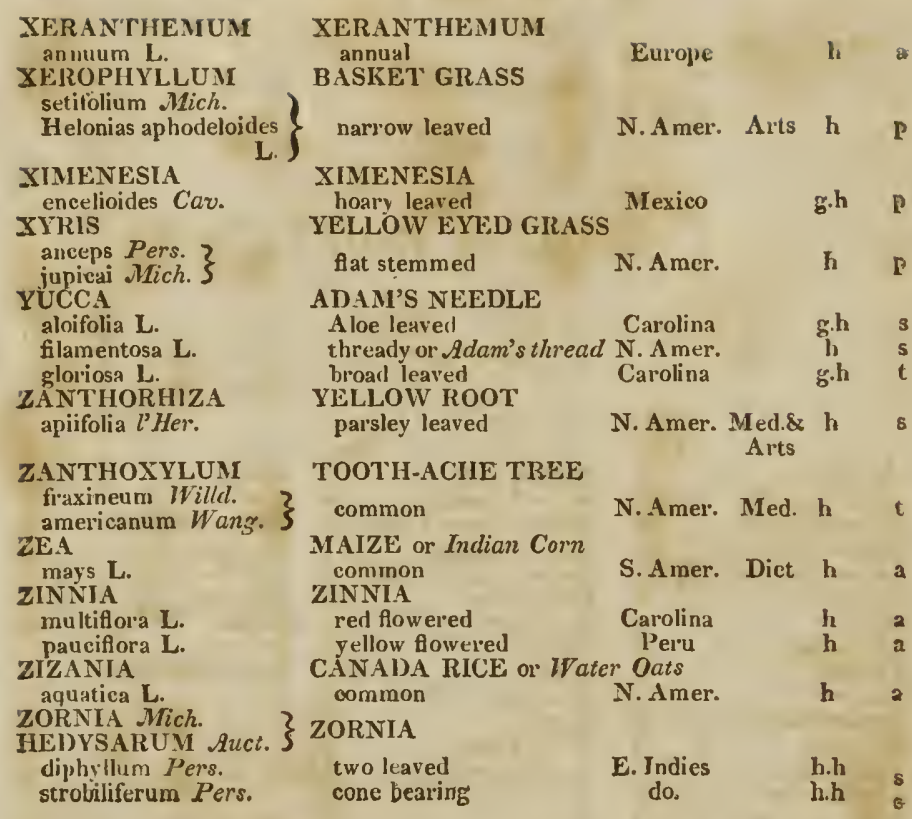


ENGLISH INDEX.

\begin{tabular}{|c|c|c|c|c|c|}
\hline & Page & & Page & & Page \\
\hline ACACIA & 1 & Bastard cress & 56 & Borage & 10) \\
\hline Adam's necdle & 60 & Bastard jasmine & 14 & Bonduc & 26 \\
\hline Ndam's thread & 60 & Bastard J esuit's & ark 30 & Botany-Bay tree & 33 \\
\hline African marygold & 56 & Bastard indigo & 4 & Bowman's root & $5+$ \\
\hline Africau sparrow wort & $\operatorname{ort} 41$ & Bastaril sensitive & plant 2 & Box thorn & 34,52 \\
\hline Agrimony & 2 & Battledore & 28 & Box tree & 11 \\
\hline Alder & 3 & 13ay-berry & 37 & Brake & 46 \\
\hline Alexander's & 53 & Bay tree & 32 & Bramble & 49 \\
\hline Almond & 5 & Bench & 23 & Bread fruit tree & 7 \\
\hline Aloe & 3 & Bead tree & 36 & Briony & 10 \\
\hline Althrea frutex & 27 & Bean & 58 & Brome grass & 10 \\
\hline A maranth & 4 & Beard grass & 5 & Broome & 54 \\
\hline A merican oil nut & 26 & Bear's breech & 1 & Broom corn & 54 \\
\hline Angelica tree & 17 & Beaver tree & .35 & Broom grass & 5 \\
\hline Anise & 43 & Bect & 9 & Broom rape & 39 \\
\hline Aniseed tree & 29 & Belladonna lily & 4 & Buckbean & 36 \\
\hline Apple & 46 & Bell flower & 12 & Buckthorn & 47 \\
\hline Apricot & 4.5 & Bellpepper & 12 & Buckwheat & $4 i$ \\
\hline bor vita & 56 & Belvedere & 14 & Bugloss & 5 \\
\hline rnotto & 10 & Benjamin tree & 32 & $13 \mathrm{u}$ & 51 \\
\hline Arrow grass & 56 & Bent grass & 2 & Burdock & 7,59 \\
\hline rrow head & 49 & Bet & 9 & Bu & 45 \\
\hline Arrow wort & 35 & Bhe & 52 & Burnet saxifinge & 43,50 \\
\hline tichoke & 19 & Bil & 57 & Burr marygold & 10 \\
\hline Asarabacea & 7 & Bindweed & 16 & reed & 54 \\
\hline $\mathrm{sh}$ & $2 i$ & Birch & 9 & rfly weed & 7 \\
\hline Ash wort & 15 & Bird pepper & 12 & & 30 \\
\hline Asphodel & 8 & Bird's foot & 39 & n wond & 13 \\
\hline Atamasco lily & 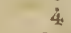 & ont trefoil & $3 \mathbf{i}$ & r's broom & 49 \\
\hline Avens & 25 & Birtlaw ort & 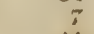 & Cabbage & 10 \\
\hline ocado pear & 32 & Bistort & 44 & Cactus & 11 \\
\hline Auricula & 4.: & Bitter apple & 18 & Cala & 2 \\
\hline $\mathrm{alm}$ & 36 & Bitter sweet & 15,53 & Camphor & 32 \\
\hline B:Ilm of Gilcad & 21,43 & Blackberry & 49 & Can & 18 \\
\hline Balsam & 29 & Black ebony & 6 & Caulada rice & 60 \\
\hline Isam apple & 37 & Black snake root & 2 & y grass & 42 \\
\hline ec & 37 & black thorn & 46 & Cancel inot & 39 \\
\hline The & ace 11 & Bladder ketıia & 27 & Canile berry myrtle & le 37 \\
\hline ruaclocs gons eberry & ty 11 & Bladiler nut & 54 & y tuft & 29 \\
\hline Barbadoes lily & 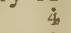 & Bladiler senna & 16 & Cape-Coast lily & 4 \\
\hline Barberry & 9 & thistle & 13 & C:tpe jasmine & 24 \\
\hline arley & 28 & Blood root & 50 & Car & 12 \\
\hline 3 ase mustard & 15 & Blue bottle & 13 & Cardinal flower & 3.3 \\
\hline Base parsley & 1.3 & yed mrass & 53 & Cariloon & 19 \\
\hline Bas & 31) & long rush & 51 & Carnation & 20 \\
\hline Jasket: & 6,0 & Bone seed & 40 & Carolina al & 11 \\
\hline Bassw or & 50 & Boneset & $\approx 3$ & Carolina jastuine & 10 \\
\hline
\end{tabular}


ENGLISH INDEX.

\begin{tabular}{|c|c|c|c|c|c|}
\hline Carolina unoss & $\begin{array}{r}\text { Page } \\
56\end{array}$ & Cotton grass & $\begin{array}{l}\text { age } \\
22\end{array}$ & Fennel & age \\
\hline Carolina or sweet pc & & Cotton thistle & 99 & Fennel fower & 38 \\
\hline tatoe & 16 & Cotton tree & 14 & Fenugreek & 57 \\
\hline Carolina pink root & 55 & Couch grass & 57 & Fern & 38 \\
\hline Carpenter's leaf & 24 & Couhage & 21 & Fescue grass & 23 \\
\hline Carrot & 20 & Cowslip & 45 & Fever-few & 46 \\
\hline Cashew nut & 5 & Cow wheat & 36 & Fever-wort & 57 \\
\hline Castor oil plant & $4 S$ & Cranberry & 58 & Field madder & 52 \\
\hline Catalpa tree & 10 & Crane's bill geranium & 24 & Field thyme & 15 \\
\hline Catch fly & 52 & Crow foot & 47 & Fig marygold & 36 \\
\hline Caterpillars & 51 & Crown asphodel & 22 & Fig tree & 23 \\
\hline Catmint & 38 & Crown imperial & 24 & Figwort & 51 \\
\hline Catnep & 38 & Cuckhold's hor'ns & $\$ 5$ & Filbert & 17 \\
\hline Cat's ear & 99 & Cucumber & 18 & Fir tree & 10 \\
\hline Cat's tail & 57 & Cucumber root & 35 & Fish poison & 55 \\
\hline Cat's tail grass & 42 & Cucumber tree & 35 & Flax & 33 \\
\hline Cedar & so & Curlweed & 25 & Flea bane & 22 \\
\hline Celandine & 14 & Currants & 48 & Flix weed & 53 \\
\hline Celery & 6 & Custard apple & 6 & Flos adonis & 2 \\
\hline entaury & 13 & Cypress tree & 18 & Flower de luce & 30 \\
\hline - & 11 & Cypress vine & 30 & Flowering fern & 40 \\
\hline docl & 15 & Dafforlil & 38 & Foxglove & 20 \\
\hline Hower & 2 & Daisy & 9 & Fox-tail grass & 4 \\
\hline omile & 6 & Dandelion & 32 & Fraxinella & 20 \\
\hline tree & 59 & Darnel & 33 & French honeysuckle & 26 \\
\hline tree & 4.5 & Date palm & 43 & Frencl marygold & 55 \\
\hline rhe & 13 & Jate plumb & 20 & Fringe tree & 14 \\
\hline ling vetch & 31 & Day & 27 & Fritillary & 24 \\
\hline Chick pea & 14 & Deadly nightshade & 8 & Fumitory & $2 \pi$ \\
\hline Chick weed & $\dot{4}, 5$ & Dead nettle & 31 & $\mathrm{Fu}$ & 59 \\
\hline Chick weed winter & & Dear grass & 47 & Galangale & 31 \\
\hline & 56 & s bit & 3 & Garden cress & 32 \\
\hline Cliin & 27 & Dewberry & 49 & Garlick & 3 \\
\hline inaclı & 0 & Dill & 6 & Gentian & 24 \\
\hline Chinquapin & 13 & Dittander & 32 & Georgia bark & 15 \\
\hline $\mathrm{Ch}$ & 3 & Dittany of Crete & 39 & Geranium & 41 \\
\hline c & 51 & Dock & 49 & Germander & 55 \\
\hline non & 32 & Dordler & 19 & Ginger & 4 \\
\hline inquefoil & 45 & Dog's bane & 6 & Ginseng & 40 \\
\hline Citron & 15 & Dog's tail grass & 19 & Glass wort & 49 \\
\hline$y^{\prime}$ & 50 & Dog's tootli violet & 22 & Globe amaranth & 25 \\
\hline rer. & 56 & Dogwood & 17 & Goat's beard & 56 \\
\hline b moss & 34 & Diagon's head & 21 & Golden rod & 53 \\
\hline t'ush & 51 & Duck's foot & 4.4 & Golden sunflower & 27 \\
\hline teat & 11 & If jaln & 49 & Golden thistle & 51 \\
\hline k's comb & $\dot{4}, 13$ & Dyer's weed & 47 & Gold of pleasure & 37 \\
\hline k's foot grass & 20 & & 24 & Gold thread & 27 \\
\hline ofiee & 16 & Egg plant & 53 & Gold tree & 8 \\
\hline e seed & 10 & Eîk & 50 & Goldy locks & 14 \\
\hline e wort & 17 & Elecampane & 29 & Gonseberry & 48 \\
\hline inticla & $1 \mathrm{~s}$ & Elephant's foot & 21 & Goose foot & 14 \\
\hline Colt's foot & 57 & Elm tree & 59 & Goose corn & 30 \\
\hline lumbine & 6 & Enchanter's night & & Gourd & 18 \\
\hline Comfrey & 55 & shade & 15 & Grape & 59 \\
\hline Contrajerva & 21 & Enclive & 14 & Grass of Parnassus & 41 \\
\hline Copaita balsam & 16 & English mercury & 14 & Green tea & 55 \\
\hline Coral and pearl & 2 & Eiryngo & 22 & Gromwell & 33 \\
\hline (1) & 22 & Eschallots & $s$ & Ground ivy & 25 \\
\hline noer & 17 & Everlasting pea & 31 & Ground-nut & 7 \\
\hline Cornelian cherry & 17 & False acacia & 48 & Groundsel & 52 \\
\hline Corn flag & 25 & False cacoa & 1 & Guava & 46 \\
\hline Costmar & 9 & Fulse grape & 3 & Guelder rose & 59 \\
\hline Cotten & 20 & Feather grass & 55 & Guernsey lily & \\
\hline
\end{tabular}


ENGLISH INDEX.

\begin{tabular}{|c|c|c|c|c|c|}
\hline & 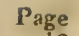 & & & & Page \\
\hline Guinea-hen weed & 42 & Jerusalem sage & 42 & Loose strife & \\
\hline Guinea pepper & 12 & Illinois nut & 30 & Lorage & 33 \\
\hline Gum arahic tree & 1 & Indian cherry tree & 22 & Love apple & 59 \\
\hline Gum tree & 33 & Indian corn & 60 & Love lies bleeding & 4 \\
\hline Hair grass & 3,56 & Indian cress & 57 & Louse wort & $\$ 1$ \\
\hline Hard grass & & Inclian fig & 11 & Lucerne & 56 \\
\hline Hare bell & 28,51 & Indian hemp & 6 & Lungwort & 46 \\
\hline Hare's ear & 11 & Indian millet & 54 & & 34 \\
\hline Hawk's beard & 18 & Indian physic & 54 & chnidea & 42 \\
\hline Hawtliorn & 17 & Indian reed & 12 & Madeira nut & so \\
\hline Hawk weed & 28 & Indian shot & 12 & $\mathrm{Ma}$ & 4 \\
\hline Hazel nut & 17 & Indian turnep & & Magothy bay bean & 12 \\
\hline $\mathbf{H}$ & 22 & Ind & 22 & hair" & 2 \\
\hline pea & 12 & Indigo wild & 44 & $M$ & 60 \\
\hline ase & 59 & Ink berry & 45 & Malabar nightshade & 9 \\
\hline hog & 38 & Job's te: & 16 & i & 31 \\
\hline ssop & 2 & & & ar plumb & 22 \\
\hline ard & 22 & anha & & & 35 \\
\hline ttle & 54 & Iro & & I wurzel & \\
\hline $\mathbf{H}$ & 27 & & & & 35 \\
\hline fern & 10 & & & & \\
\hline pruce & 43 & & 50 & & 39 \\
\hline$H$ & 1 & s beard & & ile apple & 2 \\
\hline & 28 & $\mathbf{K}$ & 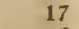 & old & 11 \\
\hline & 31 & sy coffee tre & ree & & 27 \\
\hline net & 25 & & 42 & ary & 54 \\
\hline topher & (2) & & & at & \\
\hline bert & 24 & eav & & eru & \\
\hline ass & 2 & & 29,44 & & \\
\hline Heron's bill geraniu & 22 & & 51 & & \\
\hline & 90 & & 15 & & \\
\hline ush & 58 & & 20 & saffiron & \\
\hline & 54 & d straw & 24 & grass & \\
\hline & 4 & He & 3 & & 35 \\
\hline d & 10 & & 19 & & \\
\hline & 29 & & 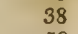 & rass & \\
\hline$k$ & 4 & & 58 & & \\
\hline & 53 & uarters & 14 & histle & 11. \\
\hline ckle & 34 & & 3 & lily & \\
\hline olt & 15 & & 20 & & \\
\hline & 2 & & 32 & ite & \\
\hline rn-beam & 4 & & 31,32 & & \\
\hline & 35 & & 58 & ass & 37 \\
\hline d water & 3 & od & 21 & & \\
\hline & 12 & & $4 \dot{4}$ & & \\
\hline 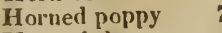 & 7,14 & $\mathbf{L}$ & 3 & ort & 14 \\
\hline & 16 & d's bane & 21 & & \\
\hline & & & 15 & uustard & 56 \\
\hline & 16 & & 5 & & \\
\hline nowe & 19 & & 31 & & ?1 \\
\hline & 52 & $\mathrm{Li}$ & 25 & & \\
\hline & 37 & Lii & & & 49 \\
\hline ng-bird & 14 & $\mathrm{Li}$ & 55 & Monkey flower & \\
\hline & & & & & \\
\hline & 29 & of the valley & 16 & & \\
\hline lily & & Lil & 13 & wood & 21 \\
\hline wil weed & 20 & & & of thyme & \\
\hline 1 & & $\mathrm{Li}$ & 50 & lother wort & \\
\hline & $\rightarrow 0$ & & 56 & in :sh & \\
\hline & 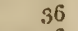 & & 3 & aij & \\
\hline & 2 & tail & & in latire & \\
\hline & 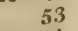 & Lo & 48 & Mountain nint & \\
\hline & 14 & L.ohlolly b\%y & $g \mathrm{fi}$ & Mrnnumain tea & \\
\hline
\end{tabular}

63

ce

33

59

4. 1

56

34

0

4

2

60

9
31

22

9

35

1

2

11

7 36

37 


\section{ENGLISH INDEA.}

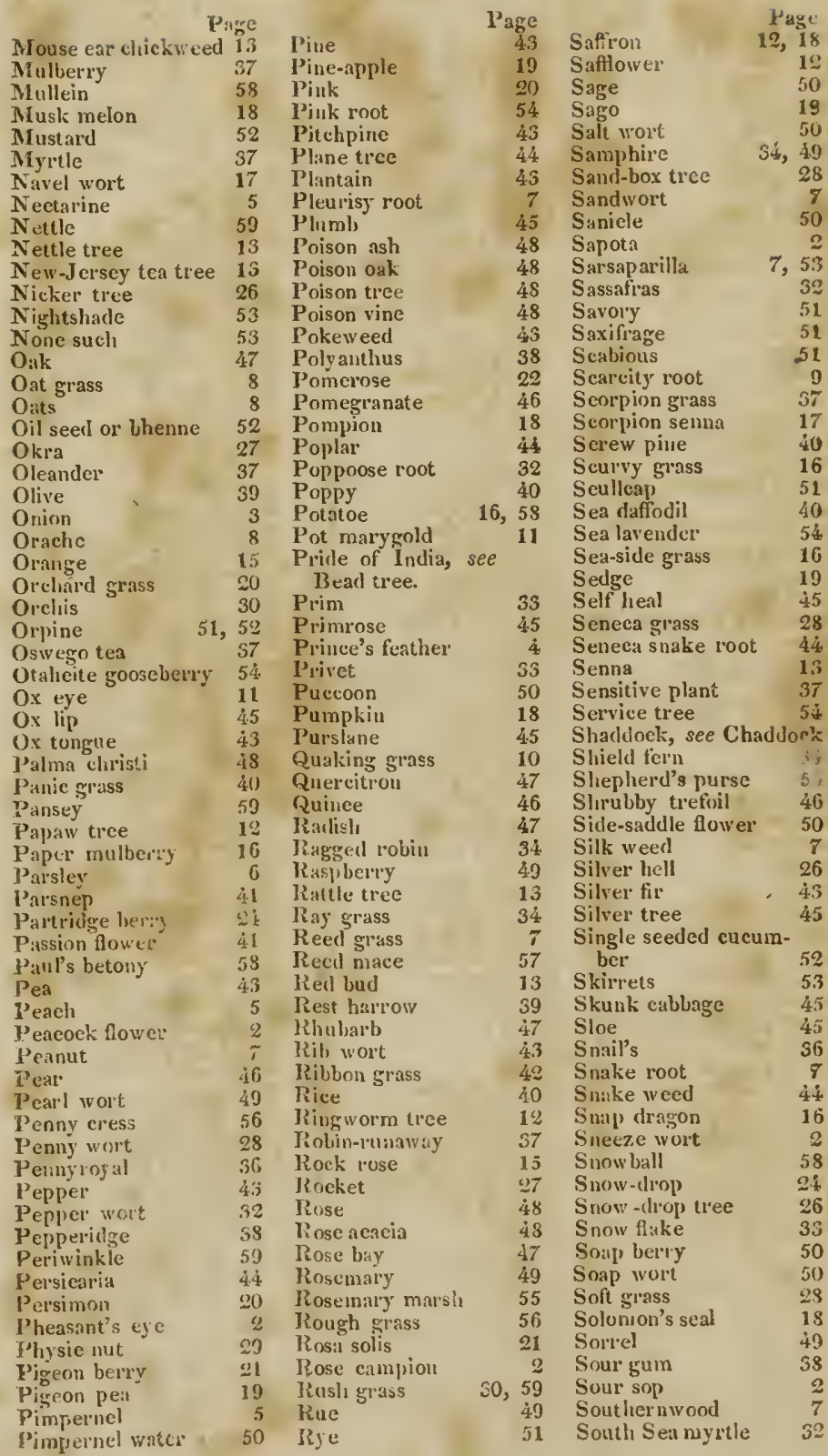




\section{ENGLISH NDEX.}

\begin{tabular}{|c|c|c|c|c|c|}
\hline & Page & as & age & & $\operatorname{agc}$ \\
\hline South Sea tea & 29 & "ramarisk & 55 & Wall flowes & 1.6 \\
\hline Sowbread & 19 & Taugier pea & 51 & WVallnut & $s+3$ \\
\hline Sow thistle & 5.3 & Tannier & 11 & Water cress & $5:-$ \\
\hline Spear wort & $4 \pi$ & Yansey & 5.3 & Water hemlock & $1 \dot{1}$ \\
\hline Speedwell & 58 & Tare & 22 & Water horehound & 36 \\
\hline Spider wort & 56 & Tartagon & 7 & Wrater lily & $x$ \\
\hline Spikenard & $i$ & Teasel & 21 & WVatermelon & 18 \\
\hline Spinach & $5 i$ & Tea tree & 55 & 1Vater oats & 60 \\
\hline Spindle tree & 22 & 12,15, & 16 & Water parsnep & 53 \\
\hline Silleen wort & 8 & Thorn apple & 20 & Water pepper & 44 \\
\hline Sjonge tree & 1 & Thorough wax & 10 & Water pimpernel & 50 \\
\hline Sprat & 28 & Tholoughwort & $2: 3$ & Water plantain & 3 \\
\hline ce & 43 & Three thorned acacia & 25 & $W^{\top}$ ater radish & 53 \\
\hline Si & 28 & Thrift & 54 & Wlieat & 57 \\
\hline ey & 54 & Thyme & 6 & eat grass & 57 \\
\hline $\mathrm{Sg}$ & 18 & rickseed sunflower & 7 & II & 52 \\
\hline So & 51 & Timothy grass & 42 & White cedar & 18,55 \\
\hline tree & 13 & Tond flax & 6 & te poplar & 33 \\
\hline apple & 14 & 'Tobacco & 38 & e wood & 33 \\
\hline uss & 3 & toes & 13 & low grass & 21 \\
\hline f Bethlehem & 39 & ca bean & 21 & tleberry & 57 \\
\hline ort & 8 & -ache tree & 60 & w wail & 1 \\
\hline estacle & 20 & h thistle & 11 & ice & 32 \\
\hline king horehound & 9 & r mustard & 57 & loss & 38 \\
\hline 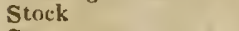 & 14 & mallow & 32 & chamomile & 35 \\
\hline crop & 51 & primrose & 39 & I chervil & 14 \\
\hline k's bill geranium & 41 & pet llower & 10 & yer & 7 \\
\hline berry & 23 & Tubeflower & 52 & Wi & \\
\hline berry blite & 10 & Tuberose & 44 & 1 indigo & 44 \\
\hline . & 14 & Tulip & 57 & Wi & 5 \\
\hline rine aloe & 5 & Tulip tree & 33 & I liquorice & \\
\hline cane & 49 & 'Tupelo tree & 38 & potatoe & 18 \\
\hline ch & 48 & eric & 19 & W & 50 \\
\hline er cypress & 14 & $\mathrm{Tu}$ & 10 & WV & 21 \\
\hline er savory & 51 & sole & 27 & W & 5 \\
\hline$v$ & 21 & flower & 56 & ar be & 4.5 \\
\hline er & 26 & $\mathrm{Va}$ & 58 & $r$ cherry & 43 \\
\hline lily & 25 & $\mathrm{Va}$ & 57 & er gaeen & 86 \\
\hline$v$ wort & 7 & ish tree & 48 & Wire grass & 14 \\
\hline p hellebore & 58 & $s^{\prime}$ comb & 51 & Witch hazel & 26 \\
\hline sassatios & 35 & Venus' fly trap & 20 & Woad & 30 \\
\hline basil & 39 & Yenus' looking-glass & 12 & Wolf's bane & \\
\hline fern & 16 & Vernal grass & 6 & Woodline & 33 \\
\hline flag & 2 & Vervain & 58 & Wool sorrel & 40 \\
\hline guin & 33 & Vetch & 5.8 & Wool griass & 22 \\
\hline pea & 31 & Vine & 59 & iI grass & 54 \\
\hline potatoc & 16 & Viulet & 59 & Worm seed & 14 \\
\hline scented slirub & 12 & Viper's bugloss & 21 & Wormwood & \\
\hline sultan & 13 & $V$ iper's grass & 51 & Yam & 20 \\
\hline -william & 20 & Virgin's hower & 15 & Yarrow & 2 \\
\hline & 42 & Uiubrella tree & 35 & Yellow eyed grass & 60 \\
\hline ahao & 坐i & Upriglt honey-suckle & 9 & Yellow root & fin \\
\hline & 55 & Walking leaf & 8 & & \\
\hline & 5.7 & Wall crees & 9 & & \\
\hline
\end{tabular}


Since the foregoing Catalogue has been printed, I have received from that distinguished Botanist, M. Thouin, Professor of Agriculture and Botany at Paris, a third collection of seeds, amounting to 300 species, of such plants as are not contained in this collection. The unceasing ex ertions of that gentleman, for the promotion of science in this country, as well as his own, deserve a greater tribute of praise than I am able to bestow.

The improvements which may hereafter take place in this institution, and the additions which may be made to the collection of plants, will in future be reguBarly published, as an annual report to the Legislature, and the Regents of the University.

D. $\mathrm{H}$. 

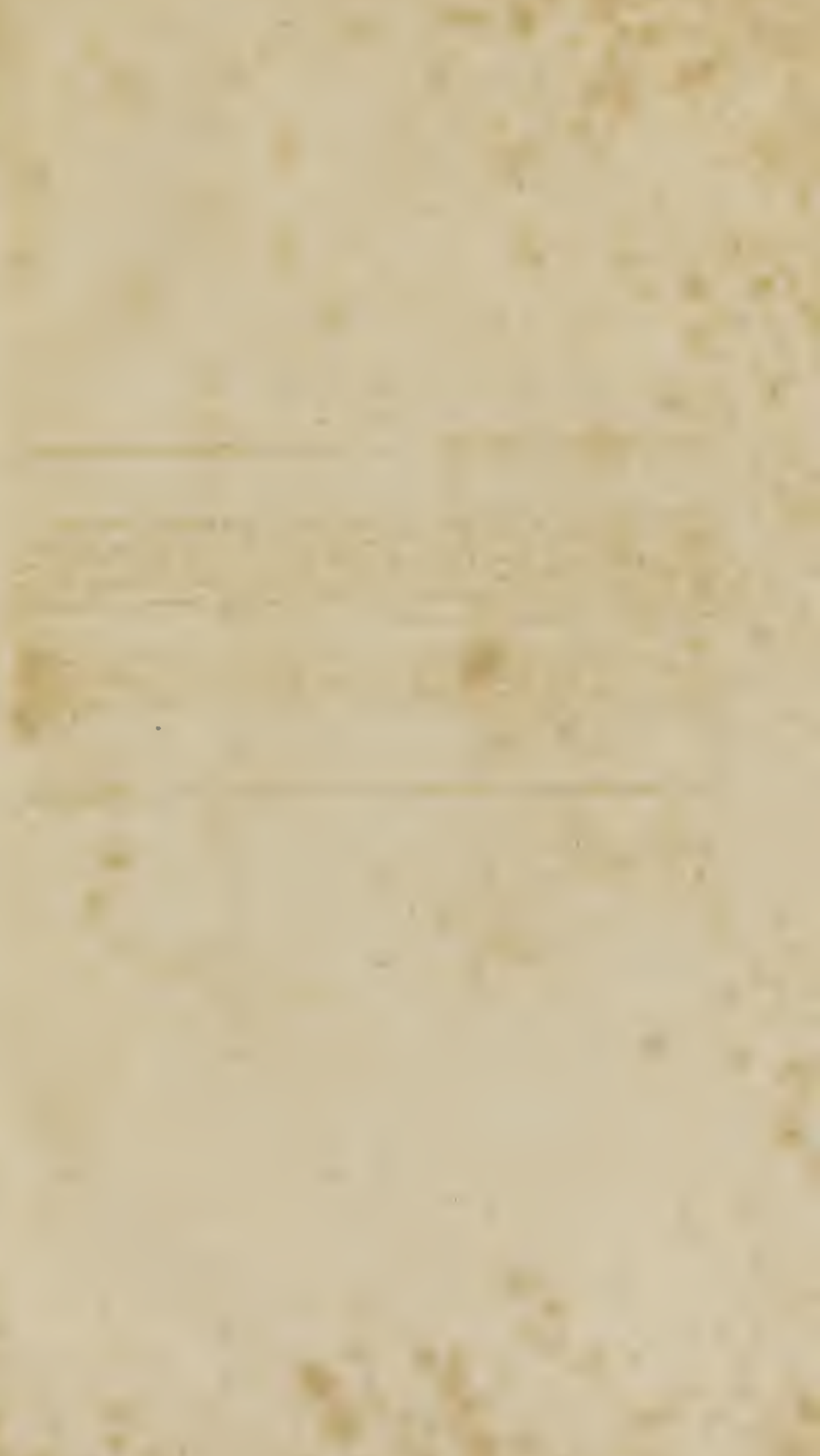

Med. Hist.

$$
\begin{gathered}
W Z \\
270 \\
4825 \text { ca.t } \\
1811 \\
\text { c.1 on }
\end{gathered}
$$


NATIONAL LIBRARY OF MEDICINE

||| |||||||||||||||||||||||| || ||||||||||| NLM 01068593 3 